
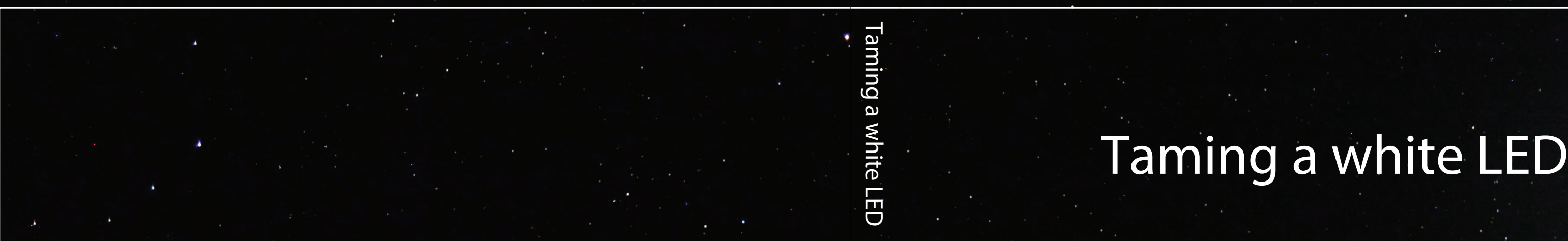

\section{Maryna Leonidivna Meretska}

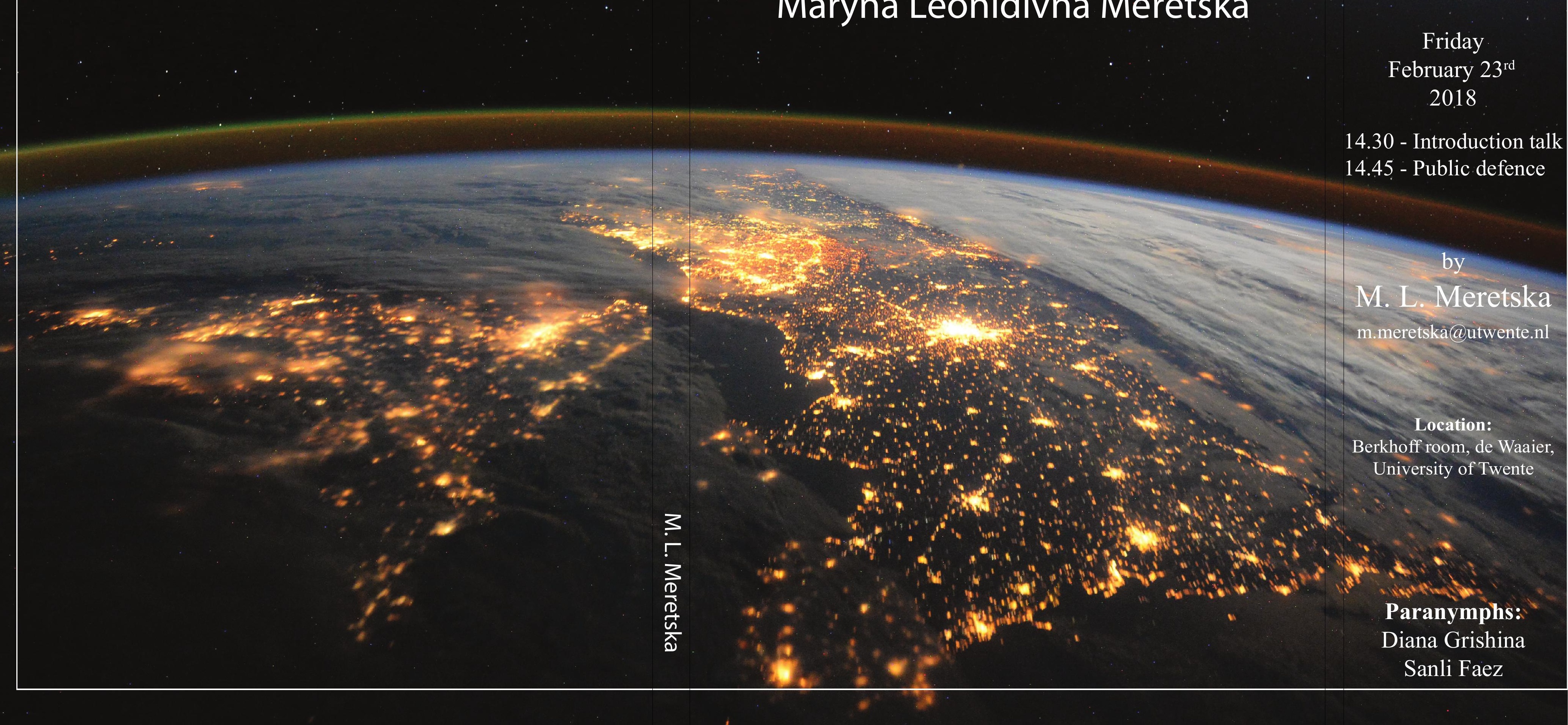

\section{Invitation \\ for}

the public defence of my $\mathrm{PhD}$ thesis title:

Taming a white LED

\section{Friday}

$23^{\text {rd }}$

2018

14.30 - Introduction talk 4.45 - Public defence

\section{khoff room, de Waaier,}

University of Twente

Paranymphs

Sanli Faez 


\title{
TAMING A WHITE LED
}

\author{
EEN WITTE LED TEMMEN
}




\section{Graduation Committee}

Chairman Prof. Dr. Ir. J. W. M. Hilgenkamp (University of Twente)

Supervisor Prof. Dr. W. L. Vos (University of Twente)

Co-supervisors $\quad$ Prof. Dr. A. Lagendijk (University of Twente)

Prof. Dr. ir. W. L. IJzerman (TU Eindhoven and Philips Lighting)

Members

Prof. Dr. K. J. Boller (University of Twente)

Prof. Dr. J. Gomez Rivas (TU Eindhoven)

Dr. J. A. Muschaweck (ARRI)

Prof. Dr. P. W. H. Pinkse (University of Twente)

Dr. R. Saive (University of Twente)

Prof. Dr. B. A. van Tiggelen (University Grenoble Alpes)

The work described in this thesis is financially supported by the STW grant '11985' and by the FOM program "Stirring of Light!".

It was carried out at the Complex Photonic Systems (COPS) chair,

Faculty of Science and Technology and MESA+ Institute for Nanotechnology, University of Twente, P.O. Box 217, 7500 AE Enschede, The Netherlands.

This thesis can be downloaded from:

http://www.cops.nano-cops.com

ISBN: 978-90-365-4490-0

Cover: ESA astronaut Tim Peake took this image of Western Europe from the International

Space Station. At the corner, one sees the Netherlands, at left the UK and right France. Courtesy of ESA. 


\title{
TAMING A WHITE LED
}

\section{PROEFSCHRIFT}

\author{
ter verkrijging van
}

de graad van doctor aan de Universiteit Twente, op gezag van de rector magnificus,

Prof. dr. T.T.M. Palstra,

volgens besluit van het College voor Promoties

in het openbaar te verdedigen

op vrijdag 23 februari 2018 om 14.45 uur

door

Maryna Leonidivna Meretska

geboren op 5 mei 1988

te Kiev, Oekraïne 
Dit proefschrift is goedgekeurd door:

Prof. Dr. W. L. Vos, promotor

Prof. Dr. A. Lagendijk, co-promotor

Prof. Dr. ir. W. L. IJzerman, co-promotor 
To my father Leonid Borysovych Meretskyi, my first teacher of physics. 


\section{Contents}

Table of Contents . . . . . . . . . . . . . . . . . . vi

List of Figures . . . . . . . . . . . . . . . . . . . . . . . . . ix

Table of symbols and notations $\ldots \ldots \ldots \ldots$. . . . . . . . . . . . .

$\begin{array}{lll}1 & \text { Introduction } & 1\end{array}$

1.1 Light . . . . . . . . . . . . . . . . . . . . . . . . . . . . . . . . . 1

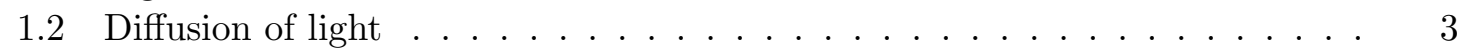

$1.2 .1 \quad$ Single particle scattering . . . . . . . . . . . . . . . . . . . 3

1.2 .2 Diffusion of light $\ldots \ldots \ldots \ldots \ldots$. . . . . . . . . . . . . 4

1.2.3 Diffusion of re-emitted light . . . . . . . . . . . . . . . 5

1.3 Outline of the thesis $\ldots \ldots \ldots \ldots \ldots \ldots$

2 Radiative transfer equation (RTE): from diffusion theory to the P3 approximation 8

2.1 Introduction $\ldots \ldots \ldots \ldots \ldots \ldots$

2.2 PN approximation $\ldots \ldots \ldots \ldots \ldots$

2.3 Collimated incident beam as a source term . . . . . . . . . . . . . . . . . . 14

2.4 Source term for the re-emitted light . . . . . . . . . . . . . . . . . . 15

2.5 P1 and P3 approximations . . . . . . . . . . . . . . . . . . . . . . 16

2.6 Boundary conditions $\ldots \ldots \ldots \ldots \ldots \ldots \ldots$

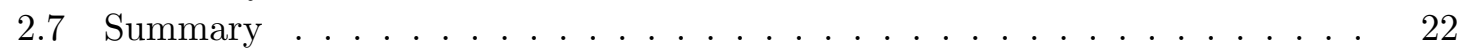

3 How to distinguish elastically scattered light from Stokes-shifted light for solid-state lighting 23

3.1 Introduction . . . . . . . . . . . . . . . . . . . . . . . . . . . . . . . 23

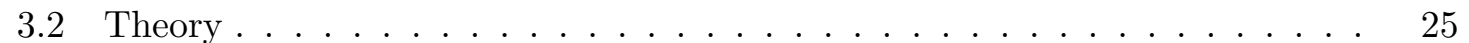

3.2 .1 Scattered transmission with energy conversion . . . . . . . . 25

3.2 .2 Scattered transmission in the absence of energy conversion . . . . . 26

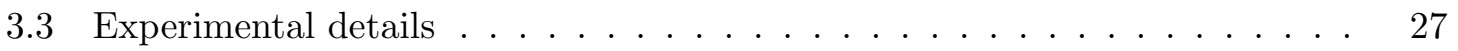

3.4 Results . . . . . . . . . . . . . . . . . . . . . . . . . . . . . . . . 30

3.4 .1 Transmission measurements . . . . . . . . . . . . . . . . . . 30

3.4 .2 Transport mean free path . . . . . . . . . . . . . . . . 33

3.4.3 Absorption mean free path . . . . . . . . . . . . . . . . 34

$3.5 \quad$ Summary and outlook $\ldots \ldots \ldots \ldots \ldots \ldots \ldots$

4 Accurate determination of transport parameters in strongly absorbing $\begin{array}{lr}\text { and scattering materials } & 39\end{array}$

4.1 Introduction $\ldots \ldots \ldots \ldots \ldots \ldots$. . . . . . . . . . . . . . . . . . . . . . 39 


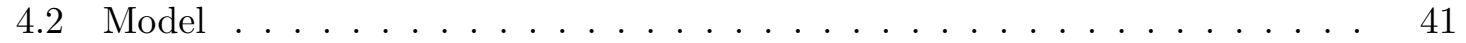

4.3 Experimental details . . . . . . . . . . . . . . . . . . . . . . . . 44

4.4 Simulations . . . . . . . . . . . . . . . . . . . . . . . . . . 46

4.5 Results . . . . . . . . . . . . . . . . . . . . . . . . . . . . 47

4.5 .1 Ballistic transmission . . . . . . . . . . . . . . . . . . 47

4.5 .2 Diffuse light transmission and reflection . . . . . . . . . . . . 48

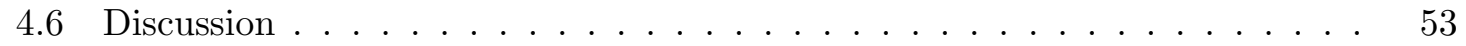

4.7 Summary and outlook $\ldots \ldots \ldots \ldots \ldots \ldots$

5 Predicting the color of white LEDs $\quad 55$

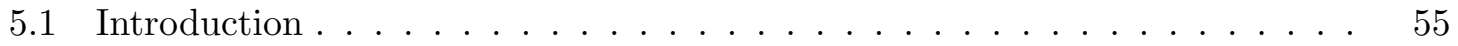

5.2 Methods . . . . . . . . . . . . . . . . . . . . 57

5.3 Results and Discussion . . . . . . . . . . . . . . . . . . . . . . . . . . . . . . . . 58

5.4 White LED with backreflector . . . . . . . . . . . . . . . . . . . . . . . 61

5.5 Summary . . . . . . . . . . . . . . . . . . . . . 65

6 Universal limit on the applicability of diffusion theory for light propaga-

$\begin{array}{ll}\text { tion } & 66\end{array}$

6.1 Introduction . . . . . . . . . . . . . . . . . . . . . . 66

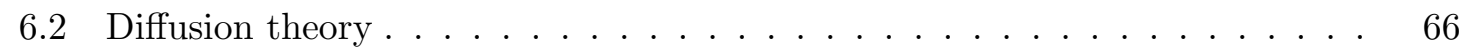

6.3 Results . . . . . . . . . . . . . . . . . . . . . . . . . . . . . 69

6.4 Error map of the diffusion theory . . . . . . . . . . . . . . . 70

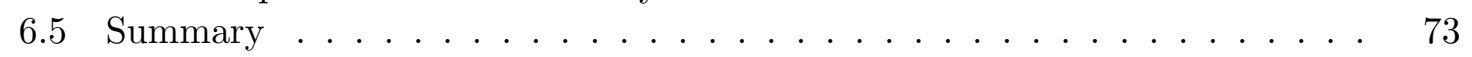

\begin{tabular}{|lll}
7 & Reversible tuning of the color point of a white LED & $\mathbf{7 5}$
\end{tabular}

7.1 Introduction . . . . . . . . . . . . . . . . . . 75

7.2 Wavefront shaping . . . . . . . . . . . . . . . . . . . 75

7.3 Setup . . . . . . . . . . . . . . . . . . . . . . . 79

7.4 Characterization of the setup . . . . . . . . . . . . . . . . . 81

7.4.1 Optimization algorithm . . . . . . . . . . . . . . . . . 81

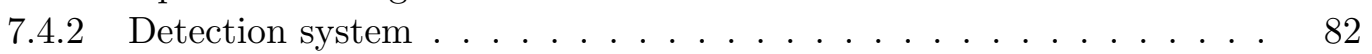

$7.4 .3 \quad$ Measurement procedure . . . . . . . . . . . . . . . . . 83

7.5 Results and discussion $\ldots \ldots \ldots \ldots$. . . . . . . . . . . . . . 85

7.6 Summary and outlook . . . . . . . . . . . . . . . . . . . . . . . . 87

\begin{tabular}{llr}
\hline 8 & Summary & 89
\end{tabular}

\begin{tabular}{ll}
\hline Nederlandse samenvatting & 92
\end{tabular}

\begin{tabular}{|ll}
\hline A Color point & 95
\end{tabular}

A.1 What is color? . . . . . . . . . . . . . . . . . . . . . . . 95

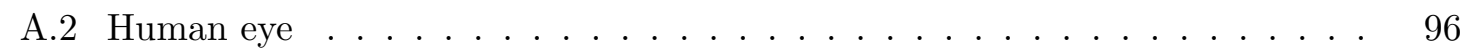

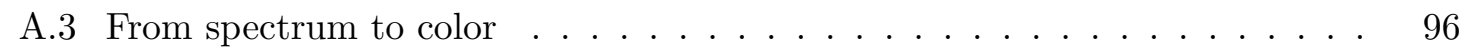

A.4 Color of objects . . . . . . . . . . . . . . . . . . . . . 100

\begin{tabular}{ll}
\hline Appendices & 95
\end{tabular}

\begin{tabular}{ll}
\hline References & 102
\end{tabular} 
\begin{tabular}{|l|l}
\hline Acknowledgments & 115
\end{tabular} 


\section{List of Figures}

$1.1 \quad$ Kyiv at night. . . . . . . . . . . . . . . . . . . . . . . . 1

$1.2 \quad$ White LED. . . . . . . . . . . . . . . . . . . . . . . . . . . . . . . 2

1.3 Scattering and diffusion of light. . . . . . . . . . . . . . . . . . . . . . 4

1.4 Scheme of the thesis. . . . . . . . . . . . . . . . . . . . 6

2.1 Coordinate space used for radiative transfer equation. . . . . . . . . . . . 9

3.2 Narrowband measurement setup. . . . . . . . . . . . . . . . . . . . . . . . 29

3.3 Normalized reference spectra of the light sources used in the experiment. . . 29

$3.4 \quad$ Flux profile of the signal that we measure in the range where emission and absorption overlap for three different pump wavelengths. . . . . . . . . . . . 30

3.5 Transport mean free path. . . . . . . . . . . . . . . . . . 32

3.6 Determination of the absorption mean free path. . . . . . . . . . . . . . . . . 34

3.7 Absorption mean free path. . . . . . . . . . . . . . . 36

$4.1 \quad$ Transport parameters in the plane spanned by absorption and diffusion. . . $\quad 40$

$4.2 \quad$ Scheme of light incident on and exiting from a slab. . . . . . . . . . . . . . 42

4.3 Experimental setup for measuring transport parameters. . . . . . . . . . . . 45

$4.4 \quad$ Ballistic transmission of the phosphor slab obtained in the experiment. . . . 47

$4.5 \quad$ Measured scattering and absorption cross sections. . . . . . . . . . . . . . 48

4.6 Transmission and scattered reflection spectra of silicone plates. . . . . . . . 49

4.7 Transmission and reflection as a function of concentration for three chosen

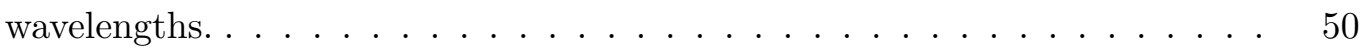

4.8 Transport parameters of YAG: $\mathrm{Ce}^{3+}$ phosphor particles extracted using analytical model and Monte Carlo simulations. . . . . . . . . . . . . 52

$5.1 \quad$ Color point of a white LED. . . . . . . . . . . . . . 56

5.2 Model for light propagation in a white LED slab. . . . . . . . . . . . . . 56

$5.3 \quad$ Representative measured signal of a model white LED. . . . . . . . . . . . . 57

5.4 Transmission and reflection as a function of phosphor particle concentration. 59

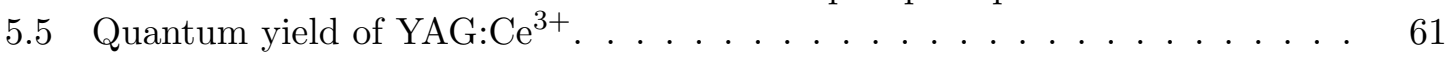

5.6 White LED with back-reflector. . . . . . . . . . . . . . . . . . . . . 62

5.7 Total transmitted flux as a function of mirror reflectivity. . . . . . . . . . . 64

6.1 Validity range of diffusion theory for a slab shown as a 2D map. . . . . . . 68

6.2 Relative error in diffusion theory calculations shown as a 2D map. . . . . . 71

6.3 Energy density (red curves) and flux (blue curves) in the unphysical range

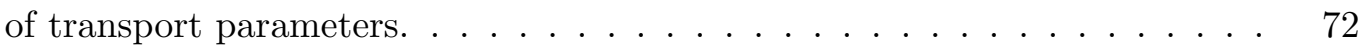


7.1 Tuning of the color point of a white LED. . . . . . . . . . . . . . . 76

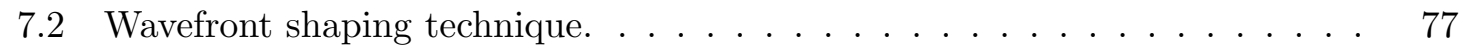

7.3 Schematic of the diffused light cone inside a slab of scattering material. . . 78

7.4 A schematic of the setup for wavefront shaping on a phosphor sample. . . . 80

7.5 The wavefront enhancement registered on the CCD as a function of the number of optimization rounds $N_{\text {run }} \ldots \ldots \ldots \ldots$. . . . . . . . . 81

$7.6 \quad$ Dark counts measurement for the experimental setup. . . . . . . . . . . 82

7.7 Calibration of the measurement setup. . . . . . . . . . . . . . . 83

7.8 Optimized spectra of the model white LED. . . . . . . . . . . . . . 84

$\begin{array}{lll}7.9 & \text { Enhancement as a function of target area for scattered and re-emitted light. } \quad 87\end{array}$

A.1 'The dress.' . . . . . . . . . . . . . . . . . . . . . . . . . . . . . . . . . . . 95

A.2 Absorbance curve of the eye sensors. . . . . . . . . . . . . . . . 96

A.3 The density of rods and cones in the eye. . . . . . . . . . . . . . . . . . 97

A.4 Hue, saturation and luminance diagram. . . . . . . . . . . . . . . . . . . 98

A.5 Color matching experiment. . . . . . . . . . . . . . . . . . . . . 99

A.6 From color space $\bar{y}$ to color space $Y . \ldots \ldots \ldots \ldots$

A.7 $\quad$ Spectrum of the white color D65. . . . . . . . . . . . . . . . . . 100

A.8 Color vs illumination conditions. . . . . . . . . . . . . . . . . . . . . . 101 


\section{Table of symbols and notations}

\begin{tabular}{|c|c|c|}
\hline Symbol & Units & Definition \\
\hline$\sigma_{\mathrm{s}}$ & $\frac{1}{\mathrm{~mm} \mathrm{wt} \% \mathrm{~Hz}}$ & scattering cross section \\
\hline$\sigma_{\mathrm{a}}$ & $\frac{1}{\mathrm{~mm} \mathrm{wt} \% \mathrm{~Hz}}$ & absorption cross section \\
\hline$\sigma_{\mathrm{t}}$ & $\frac{1}{\mathrm{~mm} \mathrm{wt} \% \mathrm{~Hz}}$ & total cross section \\
\hline$\sigma_{\mathrm{tr}}$ & $\frac{1}{\mathrm{~mm} \mathrm{wt} \% \mathrm{~Hz}}$ & transport cross section \\
\hline$\mu$ & - & anisotropy factor \\
\hline$W$ & - & single particle albedo \\
\hline$\ell_{\mathrm{S}}$ & $\mathrm{mm}$ & scattering mean free path \\
\hline$\ell_{\mathrm{a}}$ & $\mathrm{mm}$ & absorption mean free path \\
\hline$\ell_{\mathrm{tr}}$ & $\mathrm{mm}$ & transport mean free path \\
\hline$b$ & - & optical thickness of the slab \\
\hline$\lambda$ & $\mathrm{nm}$ & wavelength \\
\hline$d$ & $\mathrm{~mm}$ & thickness of the slab \\
\hline$\rho$ & $\# / \mathrm{mm}^{3}$ & number density of the scatterers \\
\hline$T_{\mathrm{tot}}^{\mathrm{rel}}(\lambda)$ & - & total relative transmission \\
\hline$T$ & - & total (elastic) transmission \\
\hline$R$ & - & total (elastic) reflection \\
\hline$T_{\mathrm{b}}$ & - & ballistic transmission \\
\hline$T_{\mathrm{r}}$ & - & re-emitted transmission \\
\hline$R_{\mathrm{r}}$ & - & re-emitted reflection \\
\hline$U_{\mathrm{d}}$ & $\frac{\mathrm{W}}{\mathrm{m}^{2} \mathrm{sr} \mathrm{Hz}}$ & diffuse average intensity \\
\hline$F_{0}$ & $\frac{\mathrm{W}}{\mathrm{m}^{2} \mathrm{~Hz}}$ & incident flux \\
\hline$\eta$ & - & enhancement \\
\hline
\end{tabular}




\section{Chapter 1}

\section{Introduction}

\section{$1.1 \quad$ Light}

Nearly all life on earth exists because of the extraterrestrial energy. Being the primary source of energy, the sun has defined and guided human lives for centuries. Even the moon would not shine in the sky if the sun disappeared or extinguished. Because the sun is so important for humans, a solar deity and sun worship can be found through most of the recorded history in many forms. A bonfire was perhaps the first artificial light source exploited by humans. Fire allowed humans to prepare food, and move around the planet at night, in brief, it greatly boosted human evolution [1].

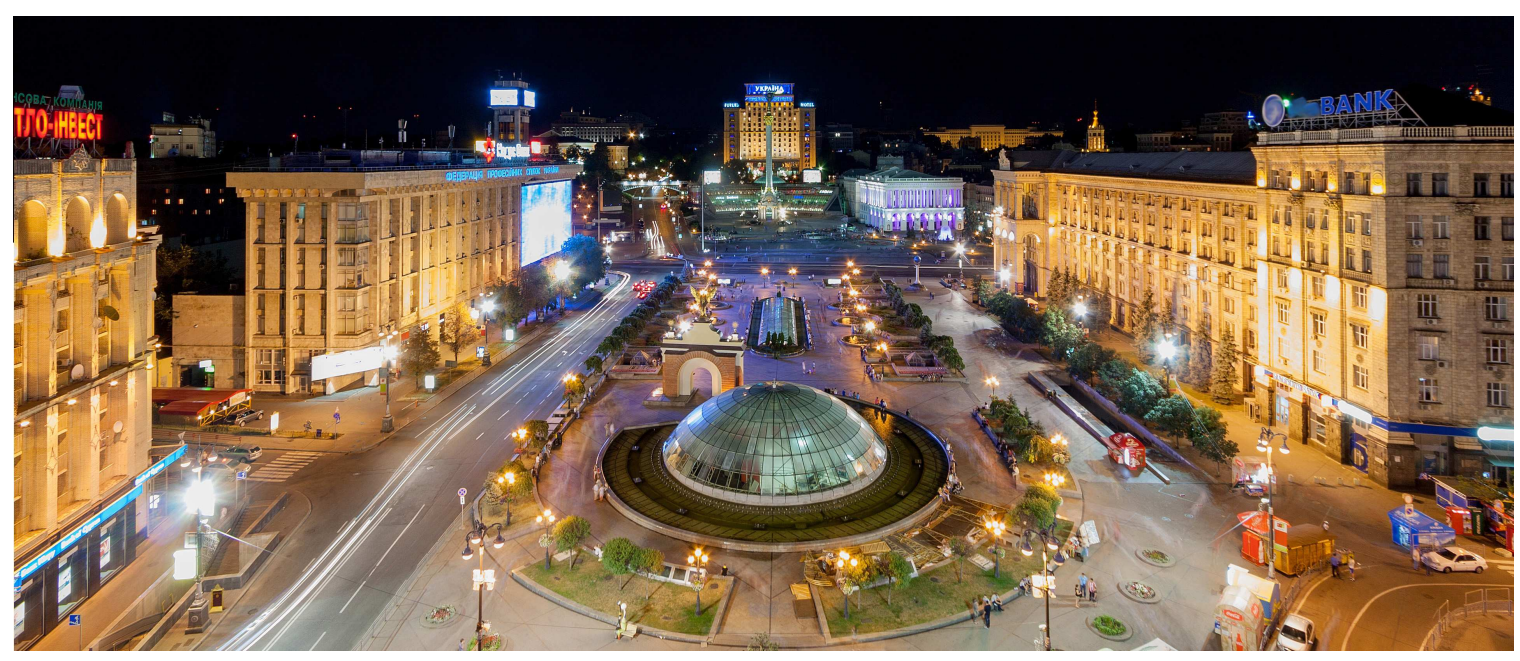

Figure 1.1: Kyiv at night. Independence square 'Maidan Nezalezhnosti' in Ukraine at night. On the left side of the glass semi-sphere in the center are the Lach gates that were one of the entrances to the city. The first mention of the gates is traced to 1151.

From fire, people moved to more advanced light sources. The first commercially available

light source that uses electricity was developed by Thomas Edison in 1878 2]. This source 
(a)

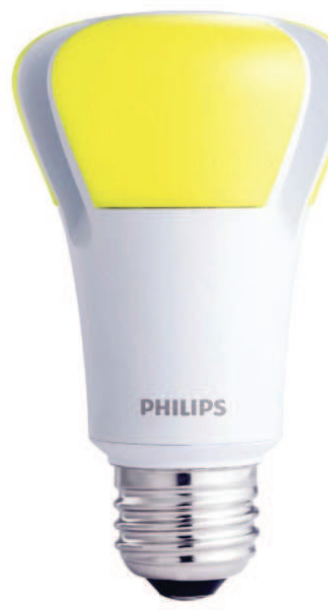

(b)

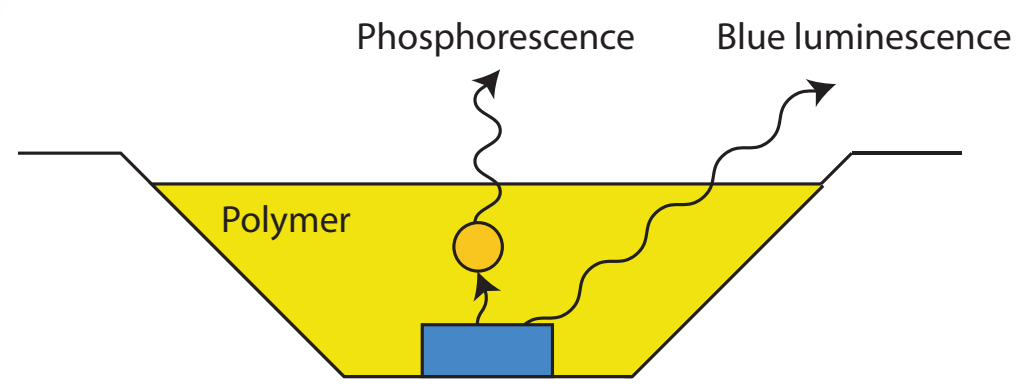

Figure 1.2: White LED. (a) L Prize winning LED lamp produced by Philips Lighting [14. (b) Schematic of the basic construction of a white LED. A cup covered with aluminum is filled with phosphor particles that are mixed with the polymer. The phosphor particles absorb blue light emitted by the blue semiconductor and re-emit light in the red and green parts of the spectrum.

was an incandescent light bulb that can be found at homes even up to now. Currently, lighting is the second most energy consuming item in everyday life [3] (see Fig. 1.1]). Since Edison's invention, the technology of white light sources has evolved tremendously. The Nobel prize-winning technology of the blue LED allowed to create energy-efficient white LEDs [4 8]. White LEDs are the most energy efficient light sources that can produce cold white light [9]. They are mechanically robust, thermally stable, possess excellent temporal stability, and have a long lifetime 9 12. A typical white LED consists of a blue semiconductor LED [5, 6, 13] in combination with a phosphor layer ,7,8 that consists of a dielectric matrix with a phosphor microparticles (see Fig. 1.2). A portion of the blue light is transmitted through the phosphor layer, and another portion is absorbed and re-emitted in the red and green part of the spectrum to yield the desired white light. The relative amount of a scattered and a re-emitted light defines the color point of a white LED that is explained in Appendix A.

In spite of the extensive use of solid-state lighting in everyday life and the apparent simplicity of the physical processes occurring in the phosphor layer, there is to date no analytical theory that predicts the spectrum of a white LED. The primary challenge arises from the lack of physical understanding of systems where multiple scattering and absorption

\footnotetext{
${ }^{1}$ The $\mathrm{L}$ Prize is a prestigious prize established by me government. This award is aimed to stimulate lighting industry in developing high-quality and high-efficiency light sources.
} 
of blue light coexist with the emission of light in a broadband wavelength range. Typically, numerical methods such as ray tracing and Monte Carlo techniques are used 15 18. These methods do not have the predictive power of analytical theory. The numerical simulations require a set of heuristic parameters that are derived from the measurements on LEDs with a wide range of structural and optical parameters. The resulting heuristic parameters are often adjusted a posteriori, thereby further hampering the predictive power of these methods. Moreover, simulations are time-consuming and computationally demanding. All these aspects obstruct the efficient design of new white LEDs.

In this thesis, I present a radically new design principle for white LEDs. In chapter 1. I introduce the basic concepts required to describe light propagation in materials that are typically used for LEDs. In section 1.2, I describe the typical transport parameters used to define multiple light scattering, absorption, and re-emission in scattering materials. The outline of the thesis is given in section 1.3 .

\subsection{Diffusion of light}

Traditionally, optics has been concerned with transparent components such as lenses, mirrors, and beam splitters [19], whereas so-called 'dirty' components that scatter light were avoided as much as possible. Over the years, however, the realization has arisen that spatial inhomogeneities that scatter light have advantageous properties and allow applications that are otherwise impossible, for instance, an optical diffuser or a high-numerical aperture objective 2022 . Both the know-how of and the control over optics that strongly scatters light has significantly advanced $[21,29]$. However, the cutting edge is much less developed regarding optical systems that also strongly absorb light (or even re-emit light of a different color), even though essential application fields occur in this regime, for instance, solid-state lighting 8, 30,31, biomedical optics [32 37], or powder technology 38 40]. In this section, I outline the transport parameters of a scattering material with absorption and re-emission that allow to describe the light propagation inside these materials.

\subsubsection{Single particle scattering}

When light illuminates a dielectric particle, part of the incident light is being scattered and absorbed by the particle (see Fig. 1.3 (a)). The amount of light that is scattered and absorbed is characterized by the scattering cross section $\sigma_{\mathrm{s}}$ and the absorption cross section

$\sigma_{\mathrm{a}}$. The scattering cross section $\sigma_{\mathrm{s}}$ is related to the total observed scattered power at all angles surrounding the particle. The absorption cross section $\sigma_{\mathrm{a}}$ is given by the total losses 
(a)

(b)

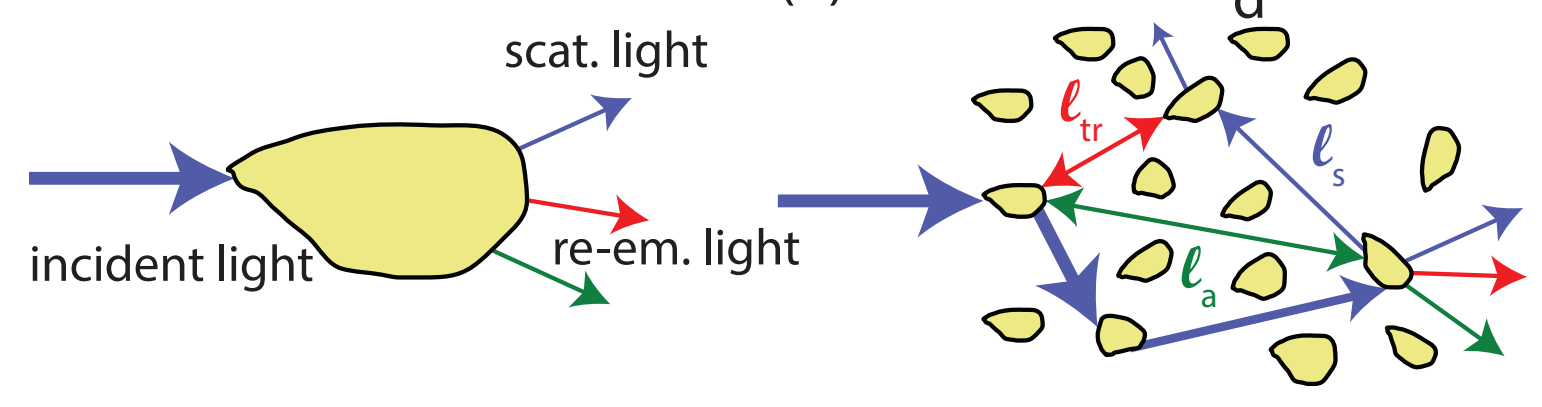

Figure 1.3: Scattering and diffusion of light. (a) A plane wave is incident upon a dielectric scatterer. The particle scatters, absorbs and re-emits incident light. (b) The multiple scattering medium with the characteristic length scales is shown. The size of the scattering medium is $d$, and the $\ell_{\mathrm{s}}, \ell_{\mathrm{tr}}, \ell_{\mathrm{a}}$ are defined in the text.

of the power inside the dielectric particle. The sum of the scattering and the absorption cross sections is called the total cross section $\sigma_{\mathrm{t}}=\sigma_{\mathrm{s}}+\sigma_{\mathrm{a}}$.

Depending on the size of the particle and the wavelength $\lambda$ of the incident light the scattering process can be divided into three classes: Rayleigh scattering, Mie scattering, and geometrical-optics scattering. When the scattering particle is much smaller than the wavelength $\lambda$, Rayleigh scattering pertains. When the scattering particle size is comparable to the wavelength $\lambda$, Mie theory describes light scattering [41]. When the particle is much larger than the wavelength $\lambda$, geometrical optics applies.

\subsubsection{Diffusion of light}

Multiple scattering of light is typically described using the set of characteristic length scales $\left(d, \ell_{\mathrm{s}}, \ell_{\mathrm{a}}, \ell_{\mathrm{tr}}\right)$ that are shown in Fig. $1.3(\mathrm{~b})$. The scattering mean free path $\ell_{\mathrm{s}}$ is the mean distance between two consecutive scattering events. The absorption mean free path $\ell_{\mathrm{a}}$ is the average diffuse propagation distance after which scattered light is absorbed by a fraction $(1 / e)$. The transport mean free path $\ell_{\operatorname{tr}}$ is the average distance after which light loses information about its original direction of propagation. These length scales are compared to the thickness of the scattering medium $d$ to gauge the scattering regime.

When the interference effects inside the sample are negligible, the radiative transfer theory (RTE) describes the light propagation inside the scattering medium [42]. Radiative transfer theory is often simplified to diffusion theory when the following inequalities hold $\ell_{\mathrm{tr}} \ll d \ll \ell_{\mathrm{a}}$ (see Ch. 2] [25]. In most of the diffuser plates used for white LEDs, this is not the case. Instead, higher order approximations to the RTE should be used, for example, the P3 approximation - the third order approximation. The input set of the parameters for 
the RTE and any order approximation is $\left(\rho, d, \sigma_{\mathrm{s}}, \sigma_{\mathrm{a}}, \mu\right)$, where $\rho$ and $\mu$ are defined below.

Under the independent scattering assumption, the simple relation between the characteristic length scales $\left(d, \ell_{\mathrm{s}}, \ell_{\mathrm{a}}, \ell_{\mathrm{tr}}\right)$ and the single particle properties $\left(\sigma_{\mathrm{s}}, \sigma_{\mathrm{a}}, \mu\right)$ can be found, where $\mu$ is the anisotropy factor that characterizes the average cosine of the scattering angle $\theta$ of the particle. Hence, the input set of the parameters for the RTE, and any order approximation is $\left(\rho, d, \sigma_{\mathrm{s}}, \sigma_{\mathrm{a}}, \mu\right)$, where $\rho$ is a number density of the scattering particles.

\subsubsection{Diffusion of re-emitted light}

In white LEDs, in addition to the scattering and absorption of light, the absorbed light is re-emitted. In the process of absorption, only a part of the incident light is being re-emitted. The rest of the energy is lost in the system in the form of heat. The efficiency of this process is characterized by the quantum yield $q\left(\lambda_{1}, \lambda_{2}\right)$, where $\lambda_{1}$ is the absorption wavelength and $\lambda_{2}$ is the emission wavelength. The quantum yield $q\left(\lambda_{1}, \lambda_{2}\right)$ is the ratio of the number of emitted photons to the number of absorbed photons.

The RTE is used to describe the propagation of the re-emitted light as in the previous case. However, I now need to know the input parameters $\left(\rho, d, \sigma_{\mathrm{s}}, \sigma_{\mathrm{a}}, \mu, q\right)$ at both the absorption and the re-emission wavelengths. Here, the absorbed light is taken to be the source of the re-emitted light with an efficiency $q$ in the RTE for the re-emitted light.

\subsection{Outline of the thesis}

In this thesis, I experimentally and theoretically study the light propagating in phosphor diffusion layers that are typically used for white LEDs. Phosphor particles inside the phosphor layer scatter, absorb, and re-emit incident light. Absorption and scattering affect light in a comparable fashion. Hence, neither of the effects can be neglected. Therefore, widely used analytical tools fail to describe phosphor diffusion layers. I propose and experimentally verify the novel systematic approach that fully describes light diffusion and re-emission in phosphor layers. The proposed method can be used to analytically model the color point of a white LED, for the first time ever.

The connections between the thesis chapters are shown in Fig. 1.4. This thesis is organized in the following way: In Ch. 2, I derive the P1 and the P3 approximation to the radiative transfer equation (RTE) using a spherical-harmonics approach, to describe the diffusion of light in the phosphor layers. To describe the re-emitted light diffusion, I derive the P3 approximation for the re-emitted light, where the source of light is the absorbed diffused light. The derived expressions are used as an analytical foundation for describing 


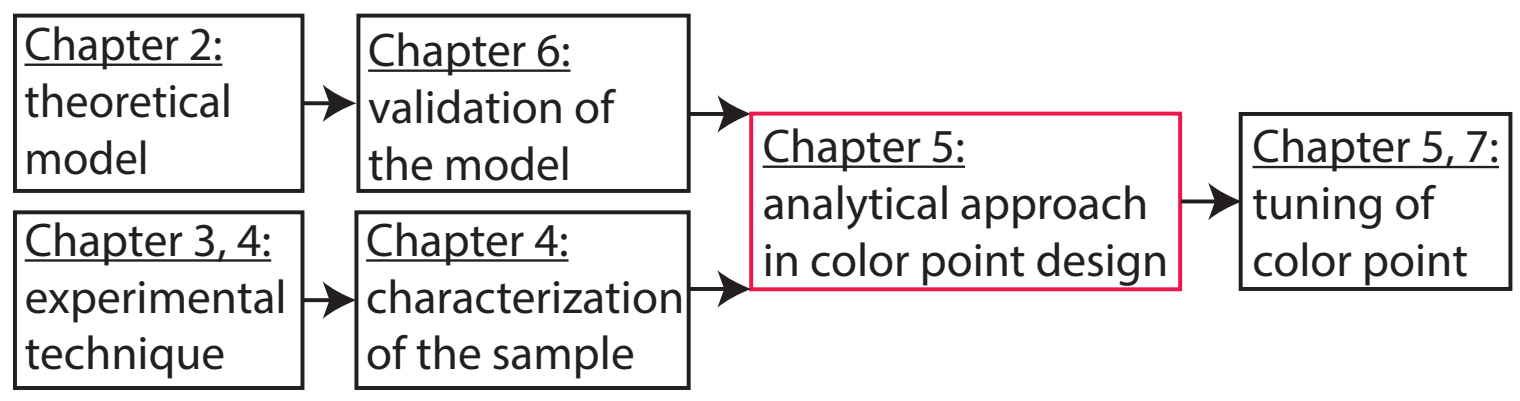

Figure 1.4: Scheme of the thesis. Arrows indicate the connections between the chapters of the thesis. The red box indicates the central question of the thesis.

light propagation and modeling color point of a white LED.

In Ch. 3, I develop the experimental technique that allows me to define transport parameters of the diffusers with strong absorption and re-emission in the whole visible spectral range. The standard experimental procedure fails in this case, because of the broadband light source that is used to measure the total transmission of the diffuser plates. When a broadband light source illuminates the diffuser with strong absorption and re-emission, the scattered and re-emitted light at the same wavelength cannot be spectrally separated. To achieve the separation of the different light components, I use a narrowband light source. A narrowband light source allows me to measure the transmission of light for the phosphor layer in the whole visible spectral range. I use diffusion theory (P1 approximation) to analyze experimental results. Diffusion theory connects measured transmission and the key transport parameters $\left(\ell_{\mathrm{tr}}, \ell_{\mathrm{a}}\right)$ of the diffuser with absorption. From experimental data, I obtain one equation and two unknowns. To resolve the arising ambiguity, I assume that the transport mean free path $\ell_{\text {tr }}$ has a linear dependence on wavelength even in the strongly absorbing wavelength range of a phosphor. This assumption allows me to determine the set $\left(\ell_{\mathrm{tr}}, \ell_{\mathrm{a}}\right)$ in the whole visible wavelength range.

In Ch. 4. I expand the experimental toolbox from Ch. 3. This expansion allows me to determine a wider set of the transport parameters $\left(\ell_{\mathrm{tr}}, \ell_{\mathrm{a}}, \mu\right)$ in the whole visible wavelength range for the scattering materials that are typically used for white LEDs. In this chapter, I use the P3 approximation to the radiative transfer equation (RTE) to analyze the experimental results. I find an excellent agreement between the transport parameters extracted using the P3 approximation and the Monte Carlo simulations. In contrast, the transport parameters extracted using the P1 approximation are off by $\sim 30 \%$. The nature of this discrepancy is described in $\mathrm{Ch}$. 6. The assumption regarding the linear dependence of the transport mean free path $\ell_{\text {tr }}$ made in Ch. 3 is confirmed.

In Ch. 5. I introduce a radically new design principle for white LEDs. My design 
principle is based on laws of physics and predicts the central characteristics of a white LED, namely its color point. The proposed method allows exploring a large parameter space as it pertains to any phosphor and any substrate used in white LED production. My approach provides a deep understanding of white LEDs and results in fast and efficient design. This method realizes a reduction of both design and production costs of solid state lighting devices. It decreases the cost of ownership of solid state lighting devices and the consumption of expensive rare earth elements used by industry and increases the efficiency of solid state lighting devices. The proposed approach requires the knowledge of the average single-particle properties listed in Sec. 1.2, and extracted in Ch. 4 .

In essence, my approach uses the P3 approximation to the RTE to describe the broadband re-emitted light. The input of the model is the set of transport parameters found in Ch. 4. The output of the model is the color of a white LED. This approach shows excellent agreement with presented experimental results and analytically maps a color point of a white LED to its spectrum. I identify the boundaries of physically achievable color points of white LED for the given design. I also experimentally extract the quantum yield $q$ for the whole visible spectrum. It allows me to describe the re-emitted light propagation correctly.

In Ch. 6, I derive the universal limit on the validity of diffusion theory for light scattering media. This chapter is motivated by the inconsistencies of the scattering parameters found in Ch. 4, when the P1 or the P3 approximations are used. I derive analytical expressions that define the region of transport parameters where diffusion theory provides physical solutions. Outside this region diffusion theory gives apparent, yet erroneous, transport parameters.

In Ch. 7, I demonstrate the reversible color tuning of the color point of a white LED. I performe optical wavefront shaping to optimize the scattered or the re-emitted light in a large area $\left(\sim 1 \mathrm{~mm}^{2}\right)$ much larger than the wavelength squared. Remarkably, theoretical estimations fail to predict any enhancement in the transmission for such large areas. When optimizing either on the scattered or the re-emitted light, the color point of the emitted light shifts. When wavefront shaping is switched off, the color point of the white LED returns to the original position. My work demonstrates the possibility to extend wavefront shaping beyond nanophotonic systems to macroscopic systems. With the help of the developed technique, I can tune the color point of a white LED beyond the designed color point. 


\section{Chapter 2}

\section{Radiative transfer equation (RTE): from diffusion theory to the P3 approximation}

\section{$2.1 \quad$ Introduction}

Transport theory deals, among others, with the transport of energy through a scattering medium. The radiative transfer equation (RTE) is the basic equation that is used in the transport theory of light [43]. Diffraction and interference effects are not included in the theory. It is assumed that there are no correlations between fields. Therefore, we consider the addition of powers rather than fields. Transport theory is equivalent to Boltzmann's equation used in the kinetic theory of gases in modern physics 43] or in the transport theory of neutrons. The radiative transfer equation is an integro-differential equation, and cannot be easily solved in the general case. In this chapter, we review the derivations of the most popular approximations such as the P1, also known as diffusion approximation, and the P3 approximation of the radiative transfer equation (RTE) in the slab geometry [43].

\section{$2.2 \quad \mathrm{PN}$ approximation}

To derive the radiative transfer equation (RTE) we consider an elementary volume with unit height and length $d s$ in space see Fig. 2.1(a). The volume $d s$ contains $\rho d s$ particles, where $\rho$ is the number density of the scattering particles. The specific intensity $I(\mathbf{r}, \hat{\mathbf{s}})$ is incident on this volume and is being scattered. The decrease of the specific intensity is written as

$$
a=\frac{d I(\mathbf{r}, \hat{\mathbf{s}})}{d s}=-\rho \sigma_{\mathrm{t}} I(\mathbf{r}, \hat{\mathbf{s}}) .
$$



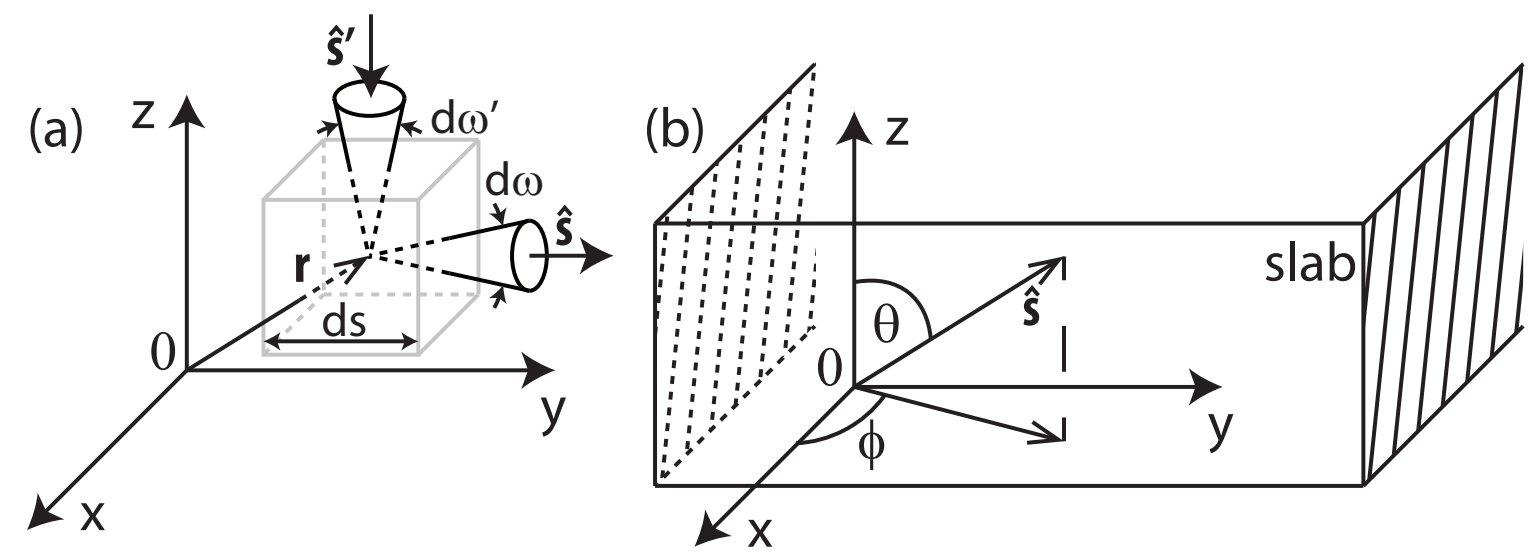

Figure 2.1: Coordinate space used for radiative transfer equation. (a) Coordinate system used in Eq. 2.3. (b) Coordinate system used in the Eq. 2.7 for the parallel slab geometry.

The specific intensity also increases when a portion of a specific intensity incident from an other direction $\hat{\mathbf{s}}^{\prime}$ is scattered into the direction $\hat{\mathbf{s}}$

$$
b=\frac{\rho \sigma_{\mathrm{t}}}{4 \pi} \int_{4 \pi} p\left(\hat{\mathbf{s}}, \hat{\mathbf{s}}^{\prime}\right) I\left(\mathbf{r}, \hat{\mathbf{s}}^{\prime}\right)
$$

where $p\left(\hat{\mathbf{s}}, \hat{\mathbf{s}}^{\prime}\right)$ is the phase function. The specific intensity can also increase due to the sources inside the medium. We sum all the contributions including the source terms to obtain the integral equation formulation of the RTE [43]:

$\frac{d I_{\mathrm{dif}}(\mathbf{r}, \hat{\mathbf{s}})}{d s}=a+b+$ sources $=-\rho \sigma_{\mathrm{t}} I_{\mathrm{dif}}(\mathbf{r}, \hat{\mathbf{s}})+\frac{\rho \sigma_{\mathrm{t}}}{4 \pi} \int_{4 \pi} p\left(\hat{\mathbf{s}}, \hat{\mathbf{s}}^{\prime}\right) I_{\mathrm{dif}}\left(\mathbf{r}, \hat{\mathbf{s}}^{\prime}\right) d \omega^{\prime}+\varepsilon_{\mathrm{ri}}(\mathbf{r}, \hat{\mathbf{s}})+\varepsilon(\mathbf{r}, \hat{\mathbf{s}})$,

where $I_{\text {dif }}(\mathbf{r}, \hat{\mathbf{s}})$ is the specific diffuse intensity (also known as the radiance or the brightness) that is the power per area per solid angle per bandwidth with units $\left[\frac{\mathrm{W}}{\mathrm{m}^{2} \mathrm{srHz}}\right], \sigma_{\mathrm{t}}$ is the total cross section, $\varepsilon(\mathbf{r}, \hat{\mathbf{s}})$ is the power generated per unit volume per unit solid angle in the direction $\hat{\mathbf{s}}$ that accounts for all sources inside the medium, $\varepsilon_{\mathrm{ri}}(\mathbf{r}, \hat{\mathbf{s}})$ is the external source function, the power generated by the reduced intensity $I_{\text {ri }}\left(\mathbf{r}, \hat{\mathbf{s}}^{\prime}\right)$

$$
\varepsilon_{\mathrm{ri}}(\mathbf{r}, \hat{\mathbf{s}})=\frac{\rho \sigma_{\mathrm{t}}}{4 \pi} \int_{4 \pi} p\left(\hat{\mathbf{s}}, \hat{\mathbf{s}}^{\prime}\right) I_{\mathrm{ri}}\left(\mathbf{r}, \hat{\mathbf{s}}^{\prime}\right) d \omega^{\prime}
$$

and all vectors used in this equation are defined in Fig. 2.1(a). Here we expanded $I(\mathbf{r}, \hat{\mathbf{s}})$ as

$$
I(\mathbf{r}, \hat{\mathbf{s}})=I_{\mathrm{ri}}(\mathbf{r}, \hat{\mathbf{s}})+I_{\text {dif }}(\mathbf{r}, \hat{\mathbf{s}}),
$$


where $I_{\text {dif }}(\mathbf{r}, \hat{\mathbf{s}})$ is the diffuse intensity and

$$
\frac{d I_{\mathrm{ri}}(\mathbf{r}, \hat{\mathbf{s}})}{d s}=-\rho \sigma_{\mathrm{t}} I_{\mathrm{ri}}(\mathbf{r}, \hat{\mathbf{s}})
$$

is the reduced incident intensity.

In the parallel slab geometry the RTE becomes

$$
\mu \frac{\partial I_{\mathrm{dif}}(z, \mu)}{\partial z}=-\rho \sigma_{\mathrm{t}} I_{\mathrm{dif}}(z, \mu)+\frac{\rho \sigma_{\mathrm{t}}}{4 \pi} \int_{4 \pi} p\left(\hat{\mathbf{s}}, \hat{\mathbf{s}}^{\prime}\right) I_{\mathrm{dif}}\left(\mathbf{r}, \hat{\mathbf{s}}^{\prime}\right) d \omega^{\prime}+\varepsilon_{\mathrm{ri}}(\mathbf{r}, \hat{\mathbf{s}})+\varepsilon(\mathbf{r}, \hat{\mathbf{s}})
$$

where we use the equality $\frac{d}{d s}=\frac{\partial}{\partial z} \cdot \frac{d z}{d s}=\cos \theta \frac{\partial}{\partial z}=\mu \frac{\partial}{\partial z}[44]$, and the coordinate system is defined in Fig. 2.1(b).

To solve the RTE analytically, we expand every term in Eq. 2.7 in the basis of spherical harmonics [45]. This expansion allows us to separate variables $\hat{\mathbf{s}}$ and $\mathbf{r}$. Additionally, in many practical problems, rotational symmetries occur that can be exploited. For example, a scattering sample is illuminated with a collimated light beam with circular cross section, or the phase function $p\left(\hat{\mathbf{s}}, \hat{\mathbf{s}}^{\prime}\right)$ depends only on the difference between the incident and the scattered angles $\mu=\cos \theta=\left.\hat{\mathbf{s}} \cdot \hat{\mathbf{s}}\right|^{1}$ [6]. Because of the symmetries, spherical harmonics are simplified to Legendre polynomials, since the dependence on the azimuth angle drops out (see Fig. 2.1(b)). As a first step, we will expand the phase function in the basis of Legendre polynomials and use addition theorem for spherical harmonics 47 to simplify the expression for the phase function $p\left(\hat{\mathbf{s}}, \hat{\mathbf{s}}^{\prime}\right)$

$$
\begin{aligned}
& p\left(\hat{\mathbf{s}}, \hat{\mathbf{s}}^{\prime}\right)=\sum_{k=0}^{K} \frac{2 k+1}{4 \pi} \mathrm{W}_{k} P_{k}\left(\hat{\mathbf{s}} \cdot \hat{\mathbf{s}}^{\prime}\right)= \\
& =\sum_{k=0}^{K} \frac{2 l+1}{4 \pi} \mathrm{W}_{k}\left[P_{k}(\mu) P_{k}\left(\mu^{\prime}\right)+\sum_{m=k}^{k} \frac{(k-m) !}{(k+m) !} P_{k}(\mu)^{m} P_{k}\left(\mu^{\prime}\right)^{m} \cos \left[m\left(\phi^{\prime}-\phi\right)\right]\right],
\end{aligned}
$$

where $\mathrm{W}_{k}$ is the $k$-th moment of the phase function $p\left(\hat{\mathbf{s}}, \hat{\mathbf{s}}^{\prime}\right), P_{l}\left(\hat{\mathbf{s}} \cdot \hat{\mathbf{s}}^{\prime}\right)$ is Legendre polynomial, $\mu=\cos \theta$ and $\mu^{\prime}=\cos \theta^{\prime}$ are the polar angles of the vectors $\hat{\mathbf{s}}$ and $\hat{\mathbf{s}}^{\prime}, \phi$ and $\phi^{\prime}$ are the azimutal angles of the vectors $\hat{\mathbf{s}}$ and $\hat{\mathbf{s}}^{\prime}$. The expansion is exact when $K \rightarrow \infty$. Further in the text, integration over the $4 \pi$ angle produces zero value for the second term in Eq. 2.8 .

\footnotetext{
${ }^{1}$ This simplification arises due to the symmetries present in the system that eliminate the dependence on the angle $\phi$.
} 
We substitute the phase function $p\left(\hat{\mathbf{s}}, \hat{\mathbf{s}}^{\prime}\right)$ from Eq. 2.8 into the third term in Eq. 2.7

$$
\begin{array}{r}
\frac{\rho \sigma_{\mathrm{t}}}{4 \pi} \int_{4 \pi} p\left(\hat{\mathbf{s}}, \hat{\mathbf{s}}^{\prime}\right) I_{\mathrm{dif}}\left(\mathbf{r}, \hat{\mathbf{s}}^{\prime}\right) d \omega^{\prime}= \\
=\frac{\rho \sigma_{\mathrm{t}}}{4 \pi} \int_{4 \pi} I_{\mathrm{dif}}\left(z, \mu^{\prime}\right) \sum_{k=0}^{K}(2 l+1) \mathrm{W}_{k} P_{k}(\mu) P_{k}\left(\mu^{\prime}\right) \sin \theta^{\prime} d \theta^{\prime} d \phi \\
=2 \pi \frac{\rho \sigma_{\mathrm{t}}}{4 \pi} \int_{-1}^{1} I_{\mathrm{dif}}\left(z, \mu^{\prime}\right) \sum_{k=0}^{K}(2 l+1) \mathrm{W}_{k} P_{k}(\mu) P_{k}\left(\mu^{\prime}\right) d \mu^{\prime} .
\end{array}
$$

In Eq. 2.9 the factor $2 \pi$ comes from the integration over $\phi$, the term proportional to $\cos \left[m\left(\phi^{\prime}-\phi\right)\right]$ vanishes due to integration [32]. Eq. 2.7 becomes

$$
\mu \frac{\partial I_{\mathrm{dif}}(z, \mu)}{\partial z}=-\rho \sigma_{\mathrm{t}} I_{\mathrm{dif}}(z, \mu)+\rho \sigma_{\mathrm{t}} \int_{-1}^{1} p\left(\mu, \mu^{\prime}\right) I_{\mathrm{dif}}\left(z, \mu^{\prime}\right) d \mu^{\prime}+\varepsilon_{\mathrm{ri}}(\mathbf{r}, \hat{\mathbf{s}})+\varepsilon(\mathbf{r}, \hat{\mathbf{s}})
$$

where

$$
p\left(\mu, \mu^{\prime}\right)=\frac{1}{2} \sum_{k=0}^{K}(2 k+1) \mathrm{W}_{k} P_{\mathrm{k}}(\mu) P_{\mathrm{k}}\left(\mu^{\prime}\right) .
$$

Next we expand the specific diffuse intensity in the basis of spherical harmonics [45]:

$$
I_{\mathrm{dif}}(\mathbf{r}, \hat{\mathbf{s}})=\sum_{l=0}^{L} \sum_{m=-l}^{l} \psi_{l m}(\mathbf{r}) Y_{l m}(\hat{\mathbf{s}})
$$

where

$$
\psi_{l m}(\mathbf{r})=\int_{4 \pi} I_{\mathrm{dif}}(\mathbf{r}, \hat{\mathbf{s}}) Y_{\operatorname{lm}}^{*}(\hat{\mathbf{s}}) d \hat{\mathbf{s}}
$$

and the spherical harmonics are defined as 48

$$
Y_{l m}(\hat{\mathbf{s}})=\sqrt{\frac{2 l+1}{4 \pi} \frac{(l-m) !}{(l+m) !}}|\hat{\mathbf{s}}| P_{l}^{m}(\cos \theta) e^{\mathrm{im} \phi},
$$

where $|\hat{\mathbf{s}}|=1, l$ is the degree of polynomial, $m$ is the order of the polynomial, and the expansion is exact when $L \rightarrow \infty$. Because of the rotational symmetries present in the system, the spherical harmonics are simplified $(m=0)$. Diffuse intensity $I_{\text {dif }}(\mathbf{r}, \hat{\mathbf{s}})$ becomes:

$$
I_{\mathrm{dif}}(\mathbf{r}, \hat{\mathbf{s}})=\sum_{l=0}^{L} \sum_{m=-l}^{l} \psi_{\operatorname{lm}=0}(\mathbf{r}) Y_{\operatorname{lm}=0}(\hat{\mathbf{s}})=\sum_{l=0}^{L} \sqrt{\frac{2 l+1}{4 \pi}} \psi_{1}(\mathbf{r}) P_{l}(\cos \theta),
$$

where in the second expression and further in the text we omit index $m$. To simplify the derivations we define

$$
I_{\mathrm{dif}}(\mathbf{r}, \hat{\mathbf{s}})=\sum_{l=0}^{L} \frac{2 l+1}{4 \pi} \psi_{\mathrm{l}}(\mathbf{r}) P_{l}(\cos \theta)
$$


where

$$
\psi_{\mathrm{l}}(\mathbf{r})=\int_{-1}^{1} I_{\mathrm{dif}}(\mathbf{r}, \hat{\mathbf{s}}) P_{l}(\mu) d \mu
$$

Until now we expanded the phase function $p\left(\hat{\mathbf{s}}, \hat{\mathbf{s}}^{\prime}\right)$ and the intensity $I_{\text {dif }}(\mathbf{r}, \hat{\mathbf{s}})$ in the Legendre polynomials basis. In the same way we expand the source term

$$
\varepsilon_{\mathrm{ri}}(\mathbf{r}, \hat{\mathbf{s}})=\sum_{l=0}^{L} \frac{2 l+1}{4 \pi} s_{l}(\mathbf{r}) P_{l}(\cos \theta)
$$

where $s_{l}$ is the $l$-th moment of the source function. We collect all the expanded terms and substitute Eq. 2.16 and Eq. 2.18 into Eq. 2.10 to obtain

$\mu \frac{\partial I_{\mathrm{dif}}(z, \mu)}{\partial z}+\sum_{l=0}^{L}\left[\rho \sigma_{\mathrm{t}} \psi_{l}(z) P_{l}(\mu)-\rho \sigma_{\mathrm{t}} \int_{-1}^{1} p\left(\mu, \mu^{\prime}\right) \psi_{l}(z) P_{l}\left(\mu^{\prime}\right) d \mu^{\prime}-s_{\mathrm{l}}(\mathbf{z}) P_{l}(\mu)\right] \frac{2 l+1}{4 \pi}=0$.

This expression is the simplified RTE 2.3. It can be simplified even further when Eq. 2.19 is multiplied by $P_{m}(\mu)$ and integrated over $2 \pi$

$$
\begin{gathered}
\int_{-1}^{1} P_{m}(\mu) \mu \frac{\partial I_{\mathrm{dif}}(z, \mu)}{\partial z} d \mu \\
+\int_{-1}^{1} d \mu \sum_{l=0}^{L} \frac{2 l+1}{4 \pi}\left[P_{m}(\mu) P_{l}(\mu) \rho \sigma_{\mathrm{t}} \psi_{l}(z)-P_{m}(\mu) \rho \sigma_{\mathrm{t}} \int_{-1}^{1} p\left(\mu, \mu^{\prime}\right) \psi_{l}(z) P_{l}\left(\mu^{\prime}\right) d \mu^{\prime}\right] \\
=\int_{-1}^{1} d \mu \sum_{l=0}^{L} \frac{2 l+1}{4 \pi}\left[P_{m}(\mu) P_{l}(\mu) s_{l}(\mathbf{z})\right] .
\end{gathered}
$$

In the next simplification step we use the following properties of the Legendre polynomials 46 . The term $P_{m}(\mu)$ in Eq. 2.9 can be expressed in the form

$$
(2 m+1) \mu P_{m}(\mu)=(m+1) P_{m+1}(\mu)+m P_{m+1}(\mu) .
$$

Another property of the Legendre polynomial that we use here is the orthogonality relation

$$
\int_{-1}^{1} P_{m}(\mu) P_{l}(\mu) d \mu=\frac{2}{2 m+1} \delta_{m l}
$$

We start simplification with the first term on the left of Eq. 2.20. Substituting Eq. 2.21 results in

$$
\begin{gathered}
\int_{-1}^{1} P_{m}(\mu) \mu \frac{\partial I_{\mathrm{dif}}(z, \mu)}{\partial z} d \mu=\int_{-1}^{1}\left(\frac{m+1}{2 m+1} P_{m+1}(\mu)+\frac{m}{2 m+1} P_{m+1}(\mu)\right) \frac{\partial I_{\mathrm{dif}}(z, \mu)}{\partial z} d \mu \\
=\frac{m+1}{2 m+1} \frac{\partial}{\partial z} \int_{-1}^{1} P_{m+1}(\mu) I_{\mathrm{dif}}(z, \mu) d \mu+\frac{m}{2 m+1} \frac{\partial}{\partial z} \int_{-1}^{1} P_{m+1}(\mu) I_{\mathrm{dif}}(z, \mu) d \mu= \\
=\frac{1}{2 \pi}\left[\frac{m+1}{2 m+1} \frac{d \psi_{m+1}(z)}{d z}+\frac{m}{2 m+1} \frac{d \psi_{m-1}(z)}{d z}\right] .
\end{gathered}
$$


For the second term in Eq. 2.20 we use Eq. 2.22

$$
\begin{gathered}
\int_{-1}^{1} d \mu \sum_{l=0}^{L} \frac{2 l+1}{4 \pi} P_{m}(\mu) P_{l}(\mu) \rho \sigma_{\mathrm{t}} \psi_{l}(z)=\sum_{l=0}^{L} \frac{2 l+1}{4 \pi} \rho \sigma_{\mathrm{t}} \psi_{l}(z) \int_{-1}^{1} P_{m}(\mu) P_{l}(\mu) d \mu \\
=\sum_{l=0}^{L} \frac{2 l+1}{4 \pi} \rho \sigma_{\mathrm{t}} \psi_{l}(z) \frac{2}{2 m+1} \delta_{m l}=\frac{1}{2 \pi} \rho \sigma_{\mathrm{t}} \psi_{\mathrm{m}}(z) .
\end{gathered}
$$

For the third term in Eq. 2.20 we use the orthogonality relation Eq. 2.22

$$
\begin{gathered}
\sum_{l=0}^{L} \frac{2 l+1}{4 \pi} \int_{-1}^{1} d \mu P_{m}(\mu) \rho \sigma_{\mathrm{t}} \int_{-1}^{1} p\left(\mu, \mu^{\prime}\right) \psi_{l}(z) P_{l}\left(\mu^{\prime}\right) d \mu^{\prime} \\
=\frac{\rho \sigma_{\mathrm{t}}}{2} \sum_{l=0}^{L} \frac{2 l+1}{4 \pi} \sum_{k=0}^{K}(2 k+1) \int_{-1}^{1} P_{m}(\mu) P_{\mathrm{k}}(\mu) d \mu \int_{-1}^{1} \mathrm{~W}_{\mathrm{k}} P_{\mathrm{k}}\left(\mu^{\prime}\right) \psi_{l}(z) P_{l}\left(\mu^{\prime}\right) d \mu^{\prime} \\
=\frac{2}{2 m+1} \frac{\rho \sigma_{\mathrm{t}}}{2} \sum_{l=0}^{L} \frac{2 l+1}{4 \pi} \sum_{k=0}^{K}(2 k+1) \delta_{\mathrm{mk}} \int_{-1}^{1} \mathrm{~W}_{\mathrm{k}} P_{\mathrm{k}}\left(\mu^{\prime}\right) \psi_{l}(z) P_{l}\left(\mu^{\prime}\right) d \mu^{\prime} \\
=\frac{2}{2 m+1} \frac{2 m+1}{2} \rho \sigma_{\mathrm{t}} \sum_{l=0}^{L} \frac{2 l+1}{4 \pi} \int_{-1}^{1} \mathrm{~W}_{\mathrm{m}} P_{\mathrm{m}}\left(\mu^{\prime}\right) \psi_{l}(z) P_{l}\left(\mu^{\prime}\right) d \mu^{\prime} \\
=\frac{2 \rho}{2 m+1} \frac{2 l+1}{4 \pi} \delta_{\mathrm{ml}} \mathrm{W}_{\mathrm{m}} \psi_{l}(z)=\frac{\rho \sigma_{\mathrm{t}}}{2 \pi} \mathrm{W}_{\mathrm{m}} \psi_{m}(z)
\end{gathered}
$$

The term on the right hand side in Eq. 2.20 is simplified with the orthogonality relation Eq. 2.22

$$
\begin{gathered}
\int_{-1}^{1} d \mu \sum_{l=0}^{L} \frac{2 l+1}{4 \pi}\left[P_{m}(\mu) P_{l}(\mu) s_{1}(\mathbf{z})\right]=\sum_{l=0}^{L} \frac{2 l+1}{4 \pi} s_{\mathrm{l}}(\mathbf{z}) \int_{-1}^{1} P_{m}(\mu) P_{l}(\mu) d \mu \\
=\frac{1}{2 \pi} s_{\mathrm{m}}(\mathbf{z}) .
\end{gathered}
$$

Adding all the simplified terms Eq. 2.23, 2.24, 2.25, 2.26 we simplify Eq. 2.20 to

$$
\frac{m+1}{2 m+1} \frac{d \psi_{m+1}(z)}{d z}+\frac{m}{2 m+1} \frac{d \psi_{m-1}(z)}{d z}+\rho \sigma_{\mathrm{t}}\left(1-\mathrm{W}_{\mathrm{m}}\right) \psi_{m}(z)-s_{\mathrm{m}}(\mathbf{z})=0
$$

where $m=0,1,2 \ldots, \infty$, and $\psi_{-1}=0$. Next, we multiply Eq. 2.27 by $2 m+1$, and arrive at the final expression

$$
(m+1) \frac{d \psi_{m+1}(z)}{d z}+m \frac{d \psi_{m-1}(z)}{d z}+\rho \sigma_{\mathrm{t}}\left(1-\mathrm{W}_{\mathrm{m}}\right)(2 m+1) \psi_{m}(z)-(2 m+1) s_{\mathrm{m}}(z)=0
$$

Expression 2.28 is the infinite set of the coupled differential equations that describe light propagation in the scattering medium. To close this system of differential equations we make the $P_{M}$ approximation, whereby we limit the expansion to order $M$. An odd positive 
integer $M$ is chosen with 35,49

$$
\psi_{\mathrm{m}}(z)=0, M<m<\infty .
$$

The $P_{M}$ approximation is usually expanded only to odd orders because even orders tend to be less accurate than the preceding odd order approximation 50,51. When $M$ is chosen we are left with a system of $M+1$ coupled first order differential equations, where $m=0,1, \ldots, M$. When $M=1$ the approximation is called $P 1$ approximation. In this case the system of two coupled first order differential equations can be transformed to one second order differential equation. This second order differential equation is called diffusion equation (see Sec. 2.5).

\subsection{Collimated incident beam as a source term}

The source term in the radiative transfer equation is

$$
\varepsilon_{\mathrm{ri}}(\mathbf{r}, \hat{\mathbf{s}})=\frac{\rho \sigma_{\mathrm{t}}}{4 \pi} \int_{4 \pi} p\left(\hat{\mathbf{s}}, \hat{\mathbf{s}}^{\prime}\right) I_{\mathrm{ri}}\left(\mathbf{r}, \hat{\mathbf{s}}^{\prime}\right) d \omega^{\prime}
$$

where the integration is performed over $4 \pi$ solid angle. Let us consider a collimated incident beam as a source term. The incident intensity of the collimated beam in 1D slab geometry is equal to

$$
I_{\mathrm{ri}}\left(\mathbf{r}, \hat{\mathbf{s}}^{\prime}\right)=F_{0} \exp \left(-\rho \sigma_{\mathrm{t}} z\right) \delta\left(\hat{\omega}^{\prime}-\hat{\omega}_{\mathrm{z}}\right)
$$

where $F_{0}$ is the incident flux, $\hat{\omega}^{\prime}$ and $\hat{\omega}_{\mathrm{z}}$ are the solid angles, and $\hat{\omega}_{\mathrm{z}}$ is aligned with the $z$

axis in Fig. 2.1(b). We insert Eq. 2.31 into Eq. 2.30 for the source term, and we integrate over the angle $\phi$ to obtain the equation

$$
\varepsilon_{\mathrm{ri}}(\mathbf{r}, \hat{\mathbf{s}})=\frac{\rho \sigma_{\mathrm{t}}}{4 \pi} 2 \pi \int_{-1}^{1} I_{\mathrm{ri}}\left(\mathbf{r}, \hat{\mathbf{s}}^{\prime}\right) \sum_{l=0}^{L}(2 l+1) \mathrm{W}_{l} P_{l}(\mu) P_{l}\left(\mu^{\prime}\right) d \mu^{\prime} .
$$


We multiply this equation by $P_{m}(\mu)$, and integrate over $2 \pi$ angle

$$
\begin{gathered}
\frac{\rho \sigma_{\mathrm{t}}}{2} \int_{-1}^{1} P_{m}(\mu) d \mu \int_{-1}^{1} I_{\mathrm{ri}}\left(\mathbf{r}, \hat{\mathbf{s}}^{\prime}\right) \sum_{l=0}^{L}(2 l+1) \mathrm{W}_{l} P_{l}(\mu) P_{l}\left(\mu^{\prime}\right) d \mu^{\prime}= \\
\frac{\rho \sigma_{\mathrm{t}}}{2} \frac{2}{2 m+1} \int_{-1}^{1} I_{\mathrm{ri}}\left(\mathbf{r}, \hat{\mathbf{s}}^{\prime}\right) \sum_{l=0}^{L}(2 l+1) \mathrm{W}_{l} P_{l}\left(\mu^{\prime}\right) \delta_{m l} d \mu^{\prime}= \\
\rho \sigma_{\mathrm{t}} \int_{-1}^{1} F_{0} \exp \left(-\rho \sigma_{\mathrm{t}} z\right) \delta\left(\hat{\omega}^{\prime}-\hat{\omega}_{\mathrm{z}}\right) \mathrm{W}_{\mathrm{m}} P_{m}\left(\mu^{\prime}\right) d \mu^{\prime}= \\
\rho \sigma_{\mathrm{t}} F_{0} \exp \left(-\rho \sigma_{\mathrm{t}} z\right) \mathrm{W}_{\mathrm{m}} \int_{-1}^{1} \delta\left(\hat{\omega}^{\prime}-\hat{\omega}_{\mathrm{z}}\right) P_{m}\left(\mu^{\prime}\right) d \mu^{\prime}= \\
\rho \sigma_{\mathrm{t}} F_{0} \exp \left(-\rho \sigma_{\mathrm{t}} z\right) \mathrm{W}_{\mathrm{m}} P_{\mathrm{m}}\left(\hat{\omega}_{\mathrm{z}}\right)=\rho \sigma_{\mathrm{t}} F_{0} \exp \left(-\rho \sigma_{\mathrm{t}} z\right) \mathrm{W}_{\mathrm{m}} P_{\mathrm{m}}(1)= \\
\rho \sigma_{\mathrm{t}} F_{0} \exp \left(-\rho \sigma_{\mathrm{t}} z\right) \mathrm{W}_{\mathrm{m}} .
\end{gathered}
$$

We substitute into Eq. 2.27 the following expression

$$
s_{m}(z)=\rho \sigma_{\mathrm{t}} F_{0} \exp \left(-\rho \sigma_{\mathrm{t}} z\right) \mathrm{W}_{m} .
$$

Coefficients $\mathrm{W}_{m}$ in Eq. 2.34 can be extracted by using the phase function $p\left(\mu, \mu^{\prime}\right) 43$

$$
p\left(\mu, \mu^{\prime}\right)=\sum_{l=0}^{L}(2 l+1) \mathrm{W}_{l} P_{l}(\mu),
$$

where

$$
\mathrm{W}_{l}=\int_{-1}^{1} p(\mu) P_{l}(\mu) d \mu
$$

We define

$$
\mathrm{p}_{1}=\frac{3}{4 \pi} \int_{4 \pi} p\left(\hat{\mathbf{s}}, \hat{\mathbf{s}}^{\prime}\right) \hat{\mathbf{s}} \cdot \hat{\mathbf{s}}^{\prime} d \hat{\mathbf{s}}^{\prime}
$$

Using Eq. 2.35 we get $\mathrm{p}_{1}=\mathrm{W}_{1}, \mu=\mathrm{p}_{1} / \mathrm{W}_{0}=\mathrm{W}_{1} / \mathrm{W}_{0}$, and finally $\mathrm{p}_{1}=\mathrm{W}_{1}=\mathrm{W}_{0} \mu$, and $\mathrm{W}_{m}=\mathrm{W}_{0} \mu^{\mathrm{m}}$, where $\mathrm{W}_{0}=\sigma_{\mathrm{s}} / \sigma_{\mathrm{t}}$ is the albedo of a single particle, and $\bar{\mu}$ is the mean cosine given by

$$
\bar{\mu}=\left(\int_{4 \pi} p\left(\hat{\mathbf{s}}, \hat{\mathbf{s}}^{\prime}\right) \mu d \hat{\mathbf{s}}^{\prime}\right) /\left(\int_{4 \pi} p\left(\hat{\mathbf{s}}, \hat{\mathbf{s}}^{\prime}\right) d \hat{\mathbf{s}}^{\prime}\right)
$$

\subsection{Source term for the re-emitted light}

In the previous section, the source of light was an reduced incident collimated beam with intensity $I_{\mathrm{ri}}\left(\mathbf{r}, \hat{\mathbf{s}}^{\prime}\right)$. In white LEDs, light is not only scattered but also absorbed and reemitted in the red and green parts of the spectra. The source function for the internally 
re-emitted light is equal to 52

$$
\varepsilon(\mathbf{r}, \hat{\mathbf{s}})=\varepsilon^{\mathrm{re}-\mathrm{em}}(\mathbf{r}, \hat{\mathbf{s}})=\frac{\rho \sigma_{\mathrm{t}}^{\mathrm{ex}}}{4 \pi} 2 \pi \int_{-1}^{1} I_{\mathrm{a}}^{\mathrm{ex}}\left(\mathbf{r}, \hat{\mathbf{s}}^{\prime}\right) \sum_{l=0}^{L}(2 l+1) \mathrm{W}_{l} P_{l}(\mu) P_{l}\left(\mu^{\prime}\right) d \mu^{\prime}
$$

where $\varepsilon^{\mathrm{re}-\mathrm{em}}(\mathbf{r}, \hat{\mathbf{s}})$ is the source function generated by the absorbed excitation light $I_{\mathrm{a}}^{\mathrm{ex}}\left(\mathbf{r}, \hat{\mathbf{s}}^{\prime}\right)$ and $\varepsilon_{\mathrm{ri}}^{\mathrm{re}-\mathrm{em}}(\mathbf{r}, \hat{\mathbf{s}})=0$. We expand $I_{\mathrm{a}}^{\mathrm{ex}}\left(\mathbf{r}, \hat{\mathbf{s}}^{\prime}\right)$ in the same way as in Eq. 2.16, and after the repeating steps from $\mathrm{Sec} .2 .2$ the source term becomes

$$
s_{\mathrm{m}}^{\mathrm{re}-\mathrm{em}}(z)=\rho \sigma_{\mathrm{t}}^{\mathrm{ex}} \mathrm{W}_{\mathrm{m}}^{\mathrm{ex}} \psi_{\mathrm{m}}^{\mathrm{ex}}(z)
$$

where $\mathrm{W}_{0}^{\mathrm{ex}}=\sigma_{\mathrm{a}}^{\mathrm{ex}} / \sigma_{\mathrm{t}}^{\mathrm{ex}}, \mathrm{W}_{\mathrm{m}}^{\mathrm{ex}}=\mathrm{W}_{0} \mu_{\mathrm{ex}}^{\mathrm{m}}=0$, the excitation light is being absorbed uniformly and hence $\mu_{\mathrm{ex}}^{\mathrm{m}}=0$. When strong absorption is present in the system (see Ch. 6), we need to consider absorbed ballistic light and absorbed scattered light. The source term function then becomes a sum of two terms

$$
s_{\mathrm{m}}^{\mathrm{re}-\mathrm{em}}(z)=\rho \sigma_{\mathrm{t}}^{\mathrm{ex}} \mathrm{W}_{\mathrm{m}}^{\mathrm{ex}}\left[\psi_{\mathrm{m}}^{\mathrm{ex}}(z)_{\mathrm{dif}}+\psi_{\mathrm{m}}^{\mathrm{ex}}(z)_{\mathrm{bal}}\right]
$$

where $\psi_{\mathrm{m}}^{\mathrm{ex}}(z)_{\mathrm{bal}}=F_{0} \exp \left(-\rho \sigma_{\mathrm{t}}^{\mathrm{ex}} z\right)$, and $\psi_{\mathrm{m}}^{\mathrm{ex}}(z)_{\text {dif }}$ is a solution of the RTE for the scattered excitation light derived in Sec. 2.2.

\subsection{P1 and P3 approximations}

Let us consider the first order approximation to the RTE, namely the P1 approximation and compare it to the diffusion approximation $U_{\mathrm{d}}(z)^{\prime \prime}-\kappa_{\mathrm{d}}^{2} U_{\mathrm{d}}(z)=-Q \exp \left(-\rho \sigma_{\mathrm{t}} z\right)^{2}$ (where $\left.U_{\mathrm{d}}(z)=\frac{1}{4 \pi} \psi_{0}\right)$ derived in 43 . We take $m=0,1$ in Eq. 2.27

$$
\begin{aligned}
\frac{d \psi_{1}}{d z}+\rho \sigma_{\mathrm{a}} \psi_{0} & =s_{0} \\
\frac{d \psi_{0}}{d z}+3 \rho \sigma_{\mathrm{tr}} \psi_{1} & =3 s_{1}
\end{aligned}
$$

where $\frac{d \psi_{2}}{d z}=0$ (from Eq. $2.29, s_{1}=\rho \sigma_{\mathrm{t}} F_{0} \exp \left(-\rho \sigma_{\mathrm{t}} z\right) \mathrm{W}_{1}$, and $s_{0}=\rho \sigma_{\mathrm{t}} F_{0} \exp \left(-\rho \sigma_{\mathrm{t}} z\right) \mathrm{W}_{0}$. This is the set of the first order coupled differential equations. From Eq. 2.43 we express $\psi_{1}$ through $\psi_{0}$ and $s_{1}$ as

$$
\psi_{1}=\frac{1}{3 \rho \sigma_{\mathrm{tr}}}\left(3 s_{1}^{\prime}-\psi_{0}^{\prime}\right)
$$

\footnotetext{
${ }^{2}$ In this section and further, symbol $/$ indicates a derivative over the spatial coordinate $z$.
} 
We substitute this relation in Eq. 2.42 to obtain

$$
\psi_{0}^{\prime \prime}-\kappa_{\mathrm{d}}^{2} \psi_{0}=-Q \exp \left(-\rho \sigma_{\mathrm{t}} z\right)
$$

where $\kappa_{\mathrm{d}}^{2}=3\left(\rho \sigma_{\mathrm{a}}\right)\left(\rho \sigma_{\mathrm{tr}}\right), Q=\left(3 \rho \sigma_{\mathrm{s}} \rho \sigma_{\mathrm{tr}}+3 \rho \sigma_{\mathrm{s}} \rho \sigma_{\mathrm{t}} \bar{\mu}\right) F_{0}$. We find back the diffusion equation from [43], when we multiply Eq. 2.45 by $1 / 4 \pi$

$$
\begin{aligned}
\frac{\partial^{2}}{\partial z^{2}}\left(\frac{1}{4 \pi} \psi_{0}\right)-\kappa_{\mathrm{d}}^{2}\left(\frac{1}{4 \pi} \psi_{0}\right) & =-\frac{1}{4 \pi} Q \exp \left(-\rho \sigma_{\mathrm{t}} z\right) \\
& \Rightarrow\left(U_{\mathrm{d}}(z)=\frac{1}{4 \pi} \psi_{0}\right) \Rightarrow \\
& \Rightarrow U_{\mathrm{d}}(z)^{\prime \prime}-\kappa_{\mathrm{d}}^{2} U_{\mathrm{d}}(z)=-Q \exp \left(-\rho \sigma_{\mathrm{t}} z\right),
\end{aligned}
$$

where we redefined $Q=\left(3 \rho \sigma_{\mathrm{s}} \rho \sigma_{\mathrm{tr}}+3 \rho \sigma_{\mathrm{s}} \rho \sigma_{\mathrm{t}} \bar{\mu}\right)\left(F_{0} / 4 \pi\right)$ in Eq. 2.47.

When higher order approximations are considered, we solve the system of differential equations differently. We illustrate this derivation method by solving the P1 approximation. We start with the system of equations 2.42 and 2.43 . There are two types of solutions for these equations: homogeneous and non-homogeneous. The non-homogeneous solution is

$$
\begin{gathered}
\psi_{0}^{\mathrm{nh}}=G_{0} \exp \left(-\rho \sigma_{\mathrm{t}} z\right), \\
\psi_{1}^{\mathrm{nh}}=G_{1} \exp \left(\rho \sigma_{\mathrm{t}} z\right),
\end{gathered}
$$

where

$$
\begin{aligned}
& G_{0}=\frac{3\left(W_{0} \gamma_{1} \rho \sigma_{\mathrm{t}}+W_{1} \rho^{2} \sigma_{\mathrm{t}}^{2}\right)}{3 \gamma_{0} \gamma_{1}-\rho^{2} \sigma_{\mathrm{t}}^{2}}, \\
& G_{1}=\frac{\rho \sigma_{\mathrm{t}}\left(3 W_{1} \gamma_{0}+W_{0} \rho \sigma_{\mathrm{t}}\right)}{3 \gamma_{0} \gamma_{1}-\rho^{2} \sigma_{\mathrm{t}}^{2}},
\end{aligned}
$$

and

$$
\begin{gathered}
\gamma_{0}=\rho \sigma_{\mathrm{a}} \\
\gamma_{1}=\rho \sigma_{\mathrm{tr}}
\end{gathered}
$$

The homogeneous solution is

$$
\begin{aligned}
& \psi_{0}=\left(H_{01}+H_{02}\right) C_{1} \exp \left(\mu_{1} z\right), \\
& \psi_{1}=\left(H_{11}+H_{12}\right) C_{2} \exp \left(\mu_{2} z\right),
\end{aligned}
$$


where

$$
\begin{aligned}
& H_{01}=1, \\
& H_{02}=1, \\
& H_{11}=-\frac{\gamma_{0}}{\mu_{1}}, \\
& H_{12}=-\frac{\gamma_{0}}{\mu_{2}},
\end{aligned}
$$

and $C_{1}, C_{2}$ are integration constants. The full solution of the diffusion approximation to the radiative transfer equation becomes

$$
\psi_{m}=\sum_{i=1}^{2} H_{m i} C_{i} \exp \left(\mu_{i} z\right)+G_{m} \exp \left(-\rho \sigma_{\mathrm{t}} z\right)
$$

for $m=0,1$, where $\mu_{i}$ is the $i$-th root of the characteristic equation for the system of differential equations 2.42 and 2.43 . The integration constants $C_{i}$ are determined from the boundary conditions (see below).

We apply this solution method to find the solution to the next approximation of the RTE, namely the P3 approximation. To obtain the P3 approximation we take $m=0,1,2,3$, and $i=1,2,3,4$ in Eq. 2.60

$$
\begin{aligned}
\frac{d \psi_{1}}{d z}+\gamma_{0} \psi_{0} & =\rho \sigma_{\mathrm{t}} F_{0} \exp \left(-\rho \sigma_{\mathrm{t}} z\right) \mathrm{W}_{0}, \\
2 \frac{d \psi_{2}}{d z}+\frac{d \psi_{0}}{d z}+3 \gamma_{1} \psi_{1} & =3 \rho \sigma_{\mathrm{t}} F_{0} \exp \left(-\rho \sigma_{\mathrm{t}} z\right) \mathrm{W}_{1} \\
3 \frac{d \psi_{3}}{d z}+2 \frac{d \psi_{1}}{d z}+5 \gamma_{2} \psi_{2} & =5 \rho \sigma_{\mathrm{t}} F_{0} \exp \left(-\rho \sigma_{\mathrm{t}} z\right) \mathrm{W}_{2}, \\
3 \frac{d \psi_{2}}{d z}+7 \gamma_{3} \psi_{3} & =7 \rho \sigma_{\mathrm{t}} F_{0} \exp \left(-\rho \sigma_{\mathrm{t}} z\right) \mathrm{W}_{3} .
\end{aligned}
$$

We take a homogeneous solution of the form

$$
\psi_{m i}(z)=H_{\mathrm{mi}} \exp \left(\mu_{\mathrm{i}} z\right)
$$

where $m=0,1,2,3$, and $i=1,2,3,4$, and insert it into Eq. 2.61, 2.62, 2.63, 2.64. The 
equations 2.61, 2.62, 2.63, 2.64) in matrix form 2.64 become

$$
\left(\begin{array}{cccc}
\gamma_{0} & \mu_{\mathrm{i}} & 0 & 0 \\
\mu_{\mathrm{i}} & 3 \gamma_{1} & 2 \mu_{\mathrm{i}} & 0 \\
0 & 2 \mu_{\mathrm{i}} & 5 \gamma_{2} & 3 \mu_{\mathrm{i}} \\
0 & 0 & 3 \mu_{\mathrm{i}} & 7 \gamma_{3}
\end{array}\right) \cdot\left(\begin{array}{l}
H_{0 i} \\
H_{1 i} \\
H_{2 i} \\
H_{3 i}
\end{array}\right)=\left(\begin{array}{l}
0 \\
0 \\
0 \\
0
\end{array}\right),
$$

where $\gamma_{\mathrm{m}}=\rho \sigma_{\mathrm{t}}\left(1-W_{m}\right)$, and $i=1,2,3,4$. The solution of the Eq. 2.75 is

$$
\begin{aligned}
& H_{0 i}=1 \\
& H_{1 i}=-\frac{\gamma_{0}}{\mu_{\mathrm{i}}} \\
& H_{2 i}=\frac{3 \gamma_{1} \gamma_{0}-\mu_{\mathrm{i}}^{2}}{2 \mu_{\mathrm{i}}^{2}} \\
& H_{3 i}=\frac{-15 \gamma_{2} \gamma_{1} \gamma_{0}+4 \gamma_{0} \mu_{\mathrm{i}}^{2}+5 \gamma_{2} \mu_{\mathrm{i}}^{2}}{6 \mu_{\mathrm{i}}^{3}}
\end{aligned}
$$

and the characteristic equation for $\mu_{\mathrm{i}}$ becomes

$$
9 \mu_{\mathrm{i}}^{4}-\mu_{\mathrm{i}}^{2}\left(35 \gamma_{2} \gamma_{3}+27 \gamma_{1} \gamma_{0}+28 \gamma_{3} \gamma_{0}\right)+105 \gamma_{0} \gamma_{1} \gamma_{2} \gamma_{3}=0
$$

The solution of Eq. 2.71 contains four distinct roots, as it is the fourth order polynomial. The solution of Eq. 2.71 is

$$
\mu_{\mathrm{i}}= \pm \sqrt{\left(b \pm \sqrt{b^{2}-3780 \gamma_{0} \gamma_{1} \gamma_{2} \gamma_{3}}\right) / 18}
$$

where $b=\left(35 \gamma_{2} \gamma_{3}+27 \gamma_{1} \gamma_{0}+28 \gamma_{3} \gamma_{0}\right)$. We take an inhomogeneous solution of the form

$$
\psi_{m}(z)=G_{\mathrm{m}} \exp \left(-\rho \sigma_{\mathrm{t}} z\right)
$$

where $m=0,1,2,3$, and insert it into Eq. 2.61, 2.62, 2.63, 2.64. In matrix form Eq. 2.61, 
$2.62,2.63,2.64$ become

$$
\left(\begin{array}{cccc}
\gamma_{0} & -\rho \sigma_{\mathrm{t}} & 0 & 0 \\
-\frac{\rho \sigma_{\mathrm{t}}}{3} & \gamma_{1} & \frac{2 \rho \sigma_{\mathrm{t}}}{3} & 0 \\
0 & -\frac{2 \rho \sigma_{\mathrm{t}}}{5} & \gamma_{2} & -\frac{3 \rho \sigma_{\mathrm{t}}}{5} \\
0 & 0 & -\frac{3 \rho \sigma_{\mathrm{t}}}{7} \mu_{\mathrm{i}} & \gamma_{3}
\end{array}\right) \cdot\left(\begin{array}{c}
G_{0} \\
G_{1} \\
G_{2} \\
G_{3}
\end{array}\right)=\left(\begin{array}{c}
\frac{\rho \sigma_{\mathrm{t}}}{2} F_{0} W_{0} \\
\frac{\rho \sigma_{\mathrm{t}}}{2} F_{0} W_{1} \\
\frac{\rho \sigma_{\mathrm{t}}}{2} F_{0} W_{2} \\
\frac{\rho \sigma_{\mathrm{t}}}{2} F_{0} W_{3}
\end{array}\right)
$$

where $\gamma_{\mathrm{m}}=\rho \sigma_{\mathrm{t}}\left(1-\frac{W_{m}}{2 m+1}\right)$. The solution of equation 2.74 becomes

$$
\left(\begin{array}{l}
G_{0} \\
G_{1} \\
G_{2} \\
G_{3}
\end{array}\right)=\left(\begin{array}{c}
\frac{F_{0} \rho \sigma_{\mathrm{t}}\left(105 \gamma_{1} \gamma_{2} \gamma_{3} W_{0}+\rho^{2} \sigma_{\mathrm{t}}^{2}\left(-27 \gamma_{1} W_{0}-28 \gamma_{3} W_{0}+70 \gamma_{3} W_{2}\right)+105 \gamma_{2} \gamma_{3} \rho \sigma_{\mathrm{t}} W_{1}+3 \rho^{3} \sigma_{\mathrm{t}}^{3}\left(14 W_{3}-9 W_{1}\right)\right)}{-2 \rho^{2} \sigma_{\mathrm{t}}^{2}\left(27 \gamma_{0} \gamma_{1}+28 \gamma_{0} \gamma_{3}+35 \gamma_{2} \gamma_{3}\right)+210 \gamma_{0} \gamma_{1} \gamma_{2} \gamma_{3}+18 \rho^{4} \sigma_{\mathrm{t}}^{4}} \\
\frac{F_{0} \rho \sigma_{\mathrm{t}}\left(-9 \rho^{3} \sigma_{\mathrm{t}}^{3} W_{0}+35 \gamma_{3} \rho \sigma_{\mathrm{t}}\left(\gamma_{2} W_{0}+2 \gamma_{0} W_{2}\right)+105 \gamma_{0} \gamma_{2} \gamma_{3} W_{1}+3 \gamma_{0} \rho^{2} \sigma_{\mathrm{t}}^{2}\left(14 W_{3}-9 W_{1}\right)\right)}{-2 \rho^{2} \sigma_{\mathrm{t}}^{2}\left(27 \gamma_{0} \gamma_{1}+28 \gamma_{0} \gamma_{3}+35 \gamma_{2} \gamma_{3}\right)+210 \gamma_{0} \gamma_{1} \gamma_{2} \gamma_{3}+18 \rho^{4} \sigma_{\mathrm{t}}^{4}} \\
\frac{7 F_{0} \rho \sigma_{\mathrm{t}}\left(\gamma_{3} \rho^{2} \sigma_{\mathrm{t}}^{2}\left(2 W_{0}-5 W_{2}\right)+3 \gamma_{0} \rho \sigma \mathrm{t}\left(2 \gamma_{3} W_{1}+3 \gamma_{1} W_{3}\right)+15 \gamma_{0} \gamma_{1} \gamma_{3} W_{2}-3 \rho^{3} \sigma_{\mathrm{t}}^{3} W_{3}\right)}{-2 \rho^{2} \sigma_{\mathrm{t}}^{2}\left(27 \gamma_{0} \gamma_{1}+28 \gamma_{0} \gamma_{3}+35 \gamma_{2} \gamma_{3}\right)+210 \gamma_{0} \gamma_{1} \gamma_{2} \gamma_{3}+18 \rho^{4} \sigma_{\mathrm{t}}^{4}} \\
\frac{F_{0} \rho \sigma_{\mathrm{t}}\left(3 \rho^{3} \sigma_{\mathrm{t}}^{3}\left(2 W_{0}-5 W_{2}\right)+\rho^{2} \sigma_{\mathrm{t}}^{2}\left(18 \gamma_{0} W_{1}-7 W_{3}\left(4 \gamma_{0}+5 \gamma_{2}\right)\right)+45 \gamma_{0} \gamma_{1} \rho \sigma_{\mathrm{t}} W_{2}+105 \gamma_{0} \gamma_{1} \gamma_{2} W_{3}\right)}{-2 \rho^{2} \sigma_{\mathrm{t}}^{2}\left(27 \gamma_{0} \gamma_{1}+28 \gamma_{0} \gamma_{3}+35 \gamma_{2} \gamma_{3}\right)+210 \gamma_{0} \gamma_{1} \gamma_{2} \gamma_{3}+18 \rho^{4} \sigma_{\mathrm{t}}^{4}}
\end{array}\right),
$$

The final solution of Eq. 2.61, 2.62, 2.63, 2.64) is obtained by combining the homogeneous and the inhomogeneous solutions.

\subsection{Boundary conditions}

In the $\mathrm{P} 1$ approximation or diffusion approximation, the net flux of light is conserved across the boundaries of the slab. This conservation law results in two boundary conditions 33

$$
\begin{aligned}
\int_{0}^{1} I_{\mathrm{dif}}(0, \mu) P_{l}(\mu) d \mu & =\int_{0}^{1} R_{2}(\mu) I_{\mathrm{dif}}(0,-\mu) P_{l}(\mu) d \mu, \\
\int_{-1}^{0} I_{\mathrm{dif}}(d,-\mu) R_{2}(\mu) P_{l}(\mu) d \mu & =\int_{-1}^{0} I_{\mathrm{dif}}(d, \mu) P_{l}(\mu) d \mu,
\end{aligned}
$$

where $l=1$, and $R_{2}(\mu)$ is the specular Fresnel reflection function for unpolarized light. We substitute the expression for intensity Eq. 2.16 in Eq. 2.77 that results in

$$
\begin{aligned}
& \sum_{m=0}^{M} \frac{2 m+1}{4 \pi} \psi_{\mathrm{m}}(0) \int_{0}^{1} P_{m}(\mu) P_{l}(\mu) d \mu=\sum_{m=0}^{M} \frac{2 m+1}{4 \pi} \psi_{\mathrm{m}}(0) \int_{0}^{1} R_{2}(\mu) P_{m}(-\mu) P_{l}(\mu) d \mu \\
& \sum_{m=0}^{M} \frac{2 m+1}{4 \pi} \psi_{\mathrm{m}}(d) \int_{-1}^{0} R_{2}(\mu) P_{m}(-\mu) P_{l}(\mu) d \mu=\sum_{m=0}^{M} \frac{2 m+1}{4 \pi} \psi_{\mathrm{m}}(d) \int_{-1}^{0} P_{m}(\mu) P_{l}(\mu) d \mu .
\end{aligned}
$$


For the P1 approximation $M=1$, and the boundary conditions 2.78 and 2.79 become

$$
\begin{gathered}
\psi_{0}(0) \int_{0}^{1} P_{0}(\mu) P_{1}(\mu)\left(1-R_{2}(\mu)\right) d \mu+3 \psi_{1}(0) \int_{0}^{1} P_{1}(\mu) P_{1}(\mu)\left(1+R_{2}(\mu)\right) d \mu=0, \\
\psi_{0}(d) \int_{-1}^{0} P_{0}(\mu) P_{1}(\mu)\left(1-R_{2}(\mu)\right) d \mu+3 \psi_{1}(d) \int_{-1}^{0} P_{1}(\mu) P_{1}(\mu)\left(1+R_{2}(\mu)\right) d \mu=0 .
\end{gathered}
$$

For the P3 approximation, we need two more equations. These additional equations are obtained by taking $l=3$ and $M=3$ in Eq. 2.78 and 2.79 .

$$
\begin{aligned}
& \sum_{m=0}^{3} \frac{2 m+1}{4 \pi} \psi_{\mathrm{m}}(0) \int_{0}^{1} P_{m}(\mu) P_{3} d \mu=\sum_{m=0}^{3} \frac{2 m+1}{4 \pi} \psi_{\mathrm{m}}(0) \int_{0}^{1} R_{2}(\mu) P_{m}(-\mu) P_{3} d \mu \\
& \sum_{m=0}^{3} \frac{2 m+1}{4 \pi} \psi_{\mathrm{m}}(d) \int_{-1}^{0} R_{2}(\mu) P_{m}(-\mu) P_{3} d \mu=\sum_{m=0}^{3} \frac{2 m+1}{4 \pi} \psi_{\mathrm{m}}(d) \int_{-1}^{0} P_{m}(\mu) P_{3} d \mu
\end{aligned}
$$

where $P_{0}=1, P_{1}=\mu, P_{2}=\left(3 \mu^{2}-1\right) / 2, P_{3}=\left(5 \mu^{3}-3 \mu\right) / 2$. The boundary conditions for the $\mathrm{P} 3$ approximation become

$$
\begin{aligned}
J_{0} \psi_{0}(0)+3 J_{1} \psi_{1}(0)+5 J_{2} \psi_{2}(0)+7 J_{3} \psi_{3}(0) & =0 \\
D_{0} \psi_{0}(d)+3 D_{1} \psi_{1}(d)+5 D_{2} \psi_{2}(d)+7 D_{3} \psi_{3}(d) & =0 \\
E_{0} \psi_{0}(0)+3 E_{1} \psi_{1}(0)+5 E_{2} \psi_{2}(0)+7 E_{3} \psi_{3}(0) & =0 \\
F_{0} \psi_{0}(d)+3 F_{1} \psi_{1}(d)+5 F_{2} \psi_{2}(d)+7 F_{3} \psi_{3}(d) & =0
\end{aligned}
$$

where

$$
\begin{array}{r}
J_{0}=-D_{0}=\int_{0}^{1} \mu(1-R(\mu)) d \mu, \\
J_{1}=D_{1}=\int_{0}^{1} \mu^{2}(1+R(\mu)) d \mu, \\
J_{2}=-D_{2}=\int_{0}^{1} \mu \frac{3 \mu^{2}-1}{2}(1-R(\mu)) d \mu, \\
J_{3}=D_{3}=\int_{0}^{1} \mu \frac{5 \mu^{3}-3 \mu}{2}(1+R(\mu)) d \mu,
\end{array}
$$


and

$$
\begin{array}{r}
E_{0}=-F_{0}=\int_{0}^{1} \frac{5 \mu^{3}-3 \mu}{2}(1-R(\mu)) d \mu, \\
E_{1}=F_{1}=\int_{0}^{1} \mu \frac{5 \mu^{3}-3 \mu}{2}(1+R(\mu)) d \mu, \\
E_{2}=-F_{2}=\int_{0}^{1} \frac{5 \mu^{3}-3 \mu}{2} \frac{3 \mu^{2}-1}{2}(1-R(\mu)) d \mu, \\
E_{3}=F_{3}=\int_{0}^{1} \frac{5 \mu^{3}-3 \mu}{2} \frac{5 \mu^{3}-3 \mu}{2}(1+R(\mu)) d \mu,
\end{array}
$$

where $R(\mu)$ is Fresnel reflection coefficient for unpolarised light.

\subsection{Summary}

In this chapter, I review two major steps in deriving the P1 and the P3 approximation in the slab geometry. The first step is to expand all the terms of the RTE in the spherical harmonics and decouple $\mathbf{r}$ and $\hat{\mathbf{s}}$. Next step is to reduce the dimensionality of the equation. I explicitly show how P1 approximation can be reduced to the well known form of the diffusion equation. The presented derivations can be found throughout the scattered literature 3233 , 35. 43 51. In this chapter, these derivations are collected and presented with the consistent notations. The derived expressions account for the boundary conditions. The expression for the re-emitted light in the P3 approximation is derived considering the solution of the P3 approximation to the scattered light as a source function. The derived expressions are used throughout this thesis. 


\section{Chapter 3}

\section{How to distinguish elastically scattered light from Stokes-shifted light for solid-state lighting}

\subsection{Introduction}

An important class of white light sources is the white LED that possess numerous advantages over conventional sources, such as incandescent lamps or discharge lamps. White LEDs are the most energy-efficient sources. They are mechanically robust and thermally stable, they possess excellent temporal stability, and a long lifetime [9, 10]. A typical white LED consists of a blue semiconductor LED in combination with a phosphor layer that consists of a dielectric matrix with the phosphor microparticles [8]. To describe the light propagation in white LEDs, we must define the transport parameters of the phosphor layer. Previously a broadband light source was applied to obtain optical properties of the diffuser plates [53], and phosphor plates [54]. This approach fails when the re-emitted light overlaps spectrally with the elastically scattered light. In Fig. 3.1(c), we show the overlap region for the plates studied here in a $x-y$ chromaticity diagram. This spectral range corresponds to the green part of the white LED spectrum where the human eye is most sensitive [55]. Here, we present a novel measurement technique that allows us to separate the elastically scattered and Stokes-shifted light in the overlap range for phosphor diffuser plates that are used in commercial white LEDs. As a result, we now close the "the green gap" in Fig. 3.1(c) and extract the relevant transport and absorption parameters for solid-state lighting in the whole visible spectral range.

We use analytical theory originating from nanophotonics, wherein propagation of light is described from first principles $23,26,56,58$. Such an ab initio theory supplies fundamental physical insights on the light propagation inside solid-state lighting device [53, 54]. 

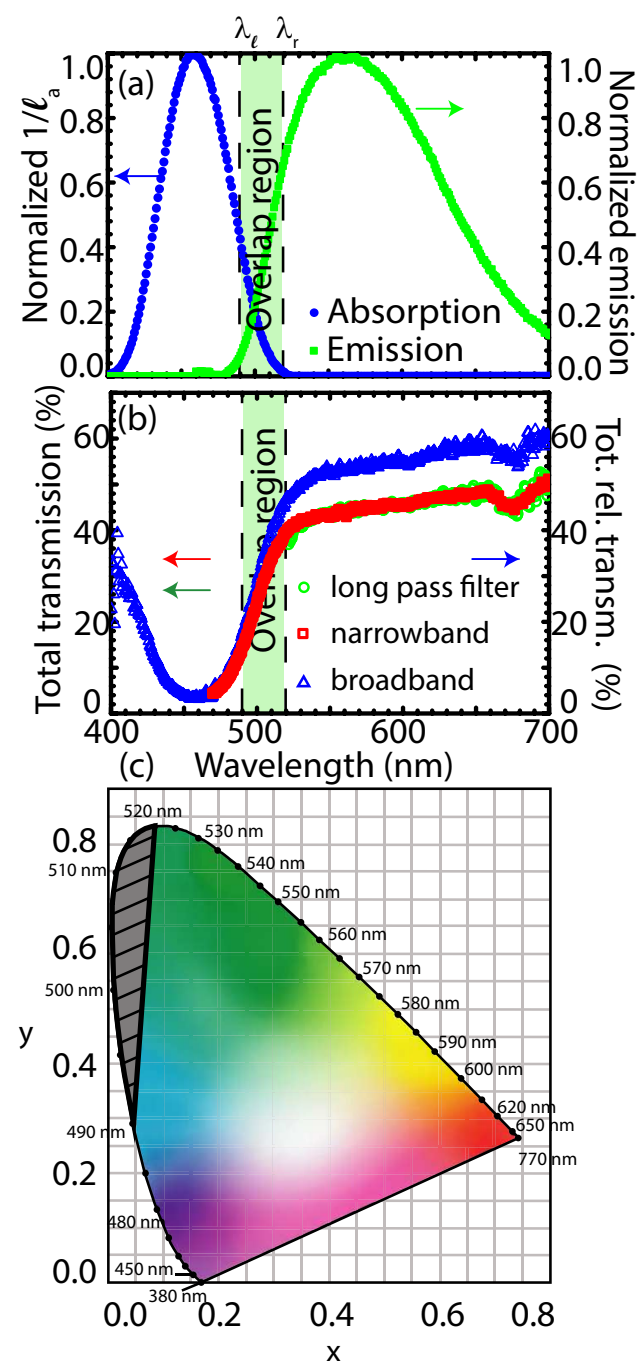

Figure 3.1: (a) Normalized absorption spectrum (blue circles) and emission spectrum (green squares) of the YAG: $\mathrm{Ce}^{3+}$ phosphor that is used in our study are shown. The spectral range where emission and absorption spectra overlap is indicated with a green bar between $\lambda_{\ell}$ and $\lambda_{\mathrm{r}}$. (b) Transmission spectrum obtained by using the narrowband (red squares) and the broadband (green circles) light sources for the polymer plate with 4 wt \% phosphor particles. Arrows point to the relevant ordinate for the data. (c) CIE 1931 $(\mathrm{x}, \mathrm{y})$ chromaticity diagram [8]. Monochromatic colors are located at the perimeter of the diagram. The white color is located in the middle of the diagram. The hatched gray area represents the region where emission and absorption of YAG:Ce ${ }^{3+}$ overlap. The overlap range was previously inaccessible, and it is made accessible in this work.

Extracting the optical parameters from theory is less time consuming than performing many simulations. More importantly: the resulting parameters are robust, and predictions can be made beyond the parameter range that was initially studied. For instance, knowledge of the absorption spectra provides us with the design guidelines for the solid-state light units. The design parameters such as the thickness of the diffuser plates and the phosphor 
concentration can be directly extracted from the absorption spectra depending on the blue pump wavelength of a white LED.

\subsection{Theory}

\subsubsection{Scattered transmission with energy conversion}

Multiple light scattering is usually studied by measuring the scattered transmission through a slab of a complex, multiple scattering medium [59,60. Scattered transmission, or diffuse transmission, is the transmission of an incident collimated beam with flux $F_{0}\left(\lambda_{\mathrm{i}}\right)$ at the incident wavelength $\lambda_{\mathrm{i}}$ that is multiply scattered and integrated over all outgoing angles of the light exiting from a medium in the forward direction. The scattered transmission carries information on the transport mean free path $\ell_{\mathrm{tr}}$ and on the absorption mean free path $\ell_{\mathrm{a}}$, which are the crucial parameters that describe multiple light scattering $[26,43,61,64]$. The transport mean free path $\ell_{\operatorname{tr}}$ is the distance it takes for the direction of light to become randomized while performing a random walk in a scattering medium. The absorption mean free path $\ell_{\mathrm{a}}$ is the distance it takes for light to be absorbed by a fraction $(1 / e)$ while light performs a random walk in a scattering medium.

Phosphor particles do not only scatter light, but also convert blue light into other colors by absorbing blue and re-emitting other colors of light. Therefore, from here on we will refer to the measured scattered transmission in presence of energy conversion as the total relative transmission $T_{\mathrm{tot}}^{\mathrm{rel}}(\lambda)$

$$
T_{\mathrm{tot}}^{\mathrm{rel}}(\lambda)=\frac{F_{\mathrm{tot}}\left(\lambda_{\mathrm{i}}\right)}{F_{0}\left(\lambda_{\mathrm{i}}\right)}
$$

where $F_{\text {tot }}\left(\lambda_{\mathrm{i}}\right)$ is the transmitted flux that is collected at the forward side of the diffusion plate. In the emission range of a phosphor $\left(\lambda \geq \lambda_{\ell}\right)$ the collected flux $F_{\text {tot }}\left(\lambda_{\mathrm{i}}\right)$ can be written as a sum of the elastic transmitted flux $F_{\mathrm{T}}\left(\lambda_{\mathrm{i}}\right)$ and the transmitted Stokes-shifted flux $F_{\text {em }}\left(\lambda_{\mathrm{i}}\right)$. Thus, the total relative transmission $T_{\mathrm{tot}}^{\mathrm{rel}}(\lambda)$ can be separated into two parts 54

$$
T_{\mathrm{tot}}^{\mathrm{rel}}\left(\lambda_{\mathrm{i}}\right)=T\left(\lambda_{\mathrm{i}}\right)+T_{\mathrm{em}}\left(\lambda_{\mathrm{i}}\right)=\frac{F\left(\lambda_{\mathrm{i}}\right)+F_{\mathrm{em}}\left(\lambda_{\mathrm{i}}\right)}{F_{0}\left(\lambda_{\mathrm{i}}\right)}
$$

where the first term $T\left(\lambda_{\mathrm{i}}\right)$ is the total elastic transmission, and the second term $T_{\mathrm{em}}\left(\lambda_{\mathrm{i}}\right)$ is the re-emitted light that experienced the energy conversion in the diffuse absorptive medium. In Ref. [54] these two terms could not be distinguished in the overlap range $\lambda_{\ell}<\lambda<\lambda_{\mathrm{r}}$.

The central question in this chapter is how to distinguish the scattered transmission $T\left(\lambda_{\mathrm{i}}\right)$ from the total relative transmission $T_{\text {tot }}^{\mathrm{rel}}$ as this allows one to obtain both the transport mean free path $\ell_{\operatorname{tr}}$ and the absorption mean free path $\ell_{\mathrm{a}}$. To access the scattered 
transmission, we employ a tunable narrowband light source and spectrally resolve the narrowband transmitted light. Since the light that is converted by the phosphor exhibits a Stokes-shift $F_{\mathrm{em}}\left(\lambda_{\mathrm{i}}\right)$, this part $T_{\mathrm{em}}\left(\lambda_{\mathrm{i}}\right)$ of the total relative transmission is filtered. Hence, we obtain the desired total elastic transmission $T\left(\lambda_{\mathrm{i}}\right)$ that we interpret with diffusion theory.

\subsubsection{Scattered transmission in the absence of energy conversion}

According to diffusion theory for light, the scattered transmission $T\left(\lambda_{\mathrm{i}}\right)$ through a slab, even in the presence of absorption, is a function of the slab thickness $d$, the wavelength $\lambda$, the transport mean free path $\ell_{\mathrm{tr}}$, and the absorption $\ell_{\mathrm{a}}$ mean free path, and can be expressed as 43$] 1$

$$
T\left(d, \lambda_{\mathrm{i}}, \ell_{\mathrm{tr}}, \ell_{\mathrm{a}}\right)=Q^{-1}\left[\sinh \left(\mu_{\mathrm{a}} z_{p}\right)+\mu_{\mathrm{a}} z_{\mathrm{e}} \cosh \left(\mu_{\mathrm{a}} z_{p}\right)\right],
$$

with

$$
Q\left(d, \lambda_{\mathrm{i}}, \ell_{\mathrm{tr}}, \ell_{\mathrm{a}}\right) \equiv\left(1+\mu_{\mathrm{a}}^{2} z_{\mathrm{e}}^{2}\right) \sinh \left(\mu_{\mathrm{a}} d\right)+2 \mu_{\mathrm{a}} z_{\mathrm{e}} \cosh \left(\mu_{\mathrm{a}} d\right),
$$

where the extrapolation lengths are equal to

$$
z_{e_{1}}\left(\ell_{\mathrm{tr}}\right)=z_{e_{2}}\left(\ell_{\mathrm{tr}}\right)=z_{\mathrm{e}}\left(\ell_{\mathrm{tr}}\right)=\frac{2}{3} \ell_{\mathrm{tr}} \frac{1+\overline{R_{1,2}}}{1-\overline{R_{1,2}}}
$$

Here $z_{p}$ is the diffuse penetration depth of light, $\mu_{\mathrm{a}} \equiv 1 / \ell_{\mathrm{a}}$ the inverse absorption mean free path, $\overline{R_{1,2}}$ is the angular and polarization averaged reflectivity of the respective boundaries [65]. For a normal incident collimated beam the penetration depth becomes $z_{p}=\ell_{\operatorname{tr}}$ 66], and $\overline{R_{1,2}}=0.57$ for polymer plates with an average refractive index $n=1.5$ from Ref. 67. For samples with no absorption $\left(\mu_{\mathrm{a}}=0\right)$, Eq. 3.3 simplifies to the optical Ohm's law 23

$$
\lim _{\ell_{\mathrm{a}} \rightarrow \infty} T\left(d, \lambda_{\mathrm{i}}, \ell_{\mathrm{tr}}, \ell_{\mathrm{a}}\right)=T\left(d, \lambda_{\mathrm{i}}, \ell_{\mathrm{tr}}\right)=\frac{\ell_{\mathrm{tr}}+z_{\mathrm{e}}}{d+2 z_{\mathrm{e}}}
$$

In the range of zero phosphor absorption, the scattered transmission is a function of the sample thickness $d$, the incoming wavelength $\lambda_{\mathrm{i}}$, and the transport mean free path $\ell_{\mathrm{tr}}, T=T\left(d, \lambda_{\mathrm{i}}, \ell_{\mathrm{tr}}\right)$. Therefore, we can extract $\ell_{\mathrm{tr}}$ using Eq. (3.6) from measurements of the scattered transmission $T\left(\lambda_{\mathrm{i}}\right)$ in the range of no absorption $\left(\lambda_{\mathrm{i}} \geq \lambda_{\mathrm{r}}\right)$. In the range of

\footnotetext{
${ }^{1}$ In Ref. 54 an approximation was used that is only valid for low absorption. Here we use the exact
expression hence our results are also valid for strong absorption (high $\mu_{\mathrm{a}}$ ). As a result, our present results
differ from Ref. 54 in the spectral range where strong absorption appears, notably near the peak of the
phosphor absorption.
} 
strong light absorption $\left(\lambda_{\mathrm{i}} \leq \lambda_{\mathrm{r}}\right)$ the scattered transmission also depends on the absorption mean free path $\ell_{\mathrm{a}}: T=T\left(d, \lambda_{\mathrm{i}}, \ell_{\mathrm{tr}}, \ell_{\mathrm{a}}\right)$. Therefore, the transport mean free path $\ell_{\mathrm{tr}}$ has to be derived separately. We extrapolate the $\ell_{\operatorname{tr}}$ values, extracted in the zero absorption wavelength range $\left(\lambda_{\mathrm{i}} \geq \lambda_{\mathrm{r}}\right)$, into the strong absorption wavelength range. It is possible because $\ell_{\operatorname{tr}}$ is a monotonously increasing function of $\lambda$ for size-polydisperse scatterers 53 , 58, 68. By measuring the scattered transmission in the range of strong absorption, we thus obtain $\ell_{\mathrm{a}}$ using extrapolated values of $\ell_{\mathrm{tr}}$, by using Eq. 3.3. The central question in this chapter is how to distinguish the scattered transmission $T\left(\lambda_{\mathrm{i}}\right)$ from the total relative transmission $T_{\text {tot }}^{\text {rel }}$ as this allows one to obtain both the transport mean free path $\ell_{\mathrm{tr}}$ and the absorption mean free path $\ell_{\mathrm{a}}$. To access the scattered transmission, we employ a tunable narrowband light source and spectrally resolve the narrowband transmitted light. Since the light that is converted by the phosphor exhibits a Stokes-shift $F_{\mathrm{em}}\left(\lambda_{\mathrm{i}}\right)$, this part $T_{\mathrm{em}}\left(\lambda_{\mathrm{i}}\right)$ of the total relative transmission is filtered. Hence, we obtain the desired total elastic transmission $T\left(\lambda_{\mathrm{i}}\right)$ that we interpret with diffusion theory.

\subsection{Experimental details}

We have studied the light transport through polymer plates that are used in Fortimo solidstate lighting units 69. The polymer plates consist of a polycarbonate matrix (Lexan 143R) with YAG: $\mathrm{Ce}^{3+}$ ceramic phosphor particles that are widely used in white LEDs. The phosphor particles have a broad diameter distribution centered around $10 \mu \mathrm{m}$ [54], and a $\mathrm{Ce}^{3+}$ concentration in the YAG:Ce ${ }^{3+}$ of $3.3 \mathrm{wt} \%$.

The emission and absorption spectra of the YAG: $\mathrm{Ce}^{3+}$ are shown in Fig. 3.1 (a) for the powder that was used in the polymer diffuser plates. The absorption and emission bands have peaked at $458 \mathrm{~nm}$ and $557 \mathrm{~nm}$, respectively, and overlap in the spectral range between $\lambda_{\ell}=490 \mathrm{~nm}$ and $\lambda_{\mathrm{r}}=520 \mathrm{~nm}$. As a result, we distinguish three spectral ranges in the visible spectrum where different physical processes are taking place: (1) in the spectral range up to $\lambda_{\ell}=490 \mathrm{~nm}$, light is partly elastically scattered and partially absorbed. This range has already been studied in Ref. [54]. (2) In the spectral range between $\lambda_{\ell}=490 \mathrm{~nm}$ and $\lambda_{\mathrm{r}}=520 \mathrm{~nm}$, incident light is elastically scattered and absorbed inside the sample. The absorbed light is re-emitted by the phosphor, and subsequently elastically scattered. This is the overlap range that is central to the present work. (3) In the spectral range beyond $\lambda_{\mathrm{r}}=520 \mathrm{~nm}$, the emitted light is the sum of the incident light that is elastically scattered and re-emitted light by the phosphor that is also elastically scattered. This range has also already been studied in Ref. [54. 
In this chapter, we present the transmission measurements on five such polymer plates with a phosphor concentration ranging from $\phi=2.0$ to $4.0 \mathrm{wt} \%$. The corresponding volume fractions range from $\phi_{\mathrm{vol}}=0.5$ to $1 \mathrm{vol} \%$, which is in the limit of low scatterer concentration because the transport mean free path is of the order of sample thickness for the given volume fractions. The plates were prepared using injection molding where a powder of YAG:Ce ${ }^{3+}$ particles is mixed with the polymer powder, and the mixture is melted and pressed into a press-form. The polymer plates, shown in Fig. 3.2(a), are circular with a diameter of 60 $\mathrm{mm}$ and a thickness of $2 \mathrm{~mm}$.

Figure 3.2 (b) shows a drawing of the setup for measuring the spectrally resolved scattered transmission $T(\lambda, d, \rho)$, where $\rho$ is the number density of the scattering particles, with a narrowband incident light beam. We illuminate the sample with two different light sources: a tunable narrowband light source and a broadband light source. The narrowband light source consists of a Fianium supercontinuum white-light source (WL-SC-UV-3), which is spectrally filtered to a bandwidth of less than $\Delta \lambda=2.4 \mathrm{~nm}$ by a prism monochromator (Carl-Leiss Berlin-Steglitz). The wavelength of the narrowband source is tunable in the wavelength range between 400 and $700 \mathrm{~nm}$, as shown in Fig 3.3. The infrared light above $700 \mathrm{~nm}$ of the supercontinuum laser source is filtered by a neutral density filter (NENIR30A) and a dichroic mirror (DMSP805). The spectrum of the supercontinuum source after filtering the infrared light is shown in Fig. 3.3 . The spectrum varies as a function of wavelength. These variations are reproducible so that we can normalize measured spectrum.

The incident beam illuminates the phosphor plate at normal incidence, and the plate is placed at the entrance port of an integrating sphere. We verified that the entrance port of the integrating sphere is sufficiently large to collect all flux that emanated from the strongest scattering sample. The flux of the outgoing light entering the integrating sphere is analyzed with a fiber-to-chip spectrometer (AvaSpec-USB2-ULS2048L) with a spectral resolution of $\Delta \lambda=2.4 \mathrm{~nm}$.

An example of the measured spectra for three incident wavelengths $\lambda_{\mathrm{i}}=527,550$ and $634 \mathrm{~nm}$ are shown in Fig. 3.3 with the red squares and red dotted lines. The peak flux varies significantly across the spectral range as a result of the spectral variation of the supercontinuum source. To maintain a fixed reference flux, we automatically adjust the integration time at every wavelength during measurements.

The alternative broadband light source consist of a white LED (Luxeon LXHL-MW1D) (see Fig. 3.2) with an emission spectrum covering the range from $400 \mathrm{~nm}$ to $700 \mathrm{~nm}$ as shown in Fig. 3.3. This source is not filtered.

For all phosphor plates, we measured the transmission spectra with both the narrow- 


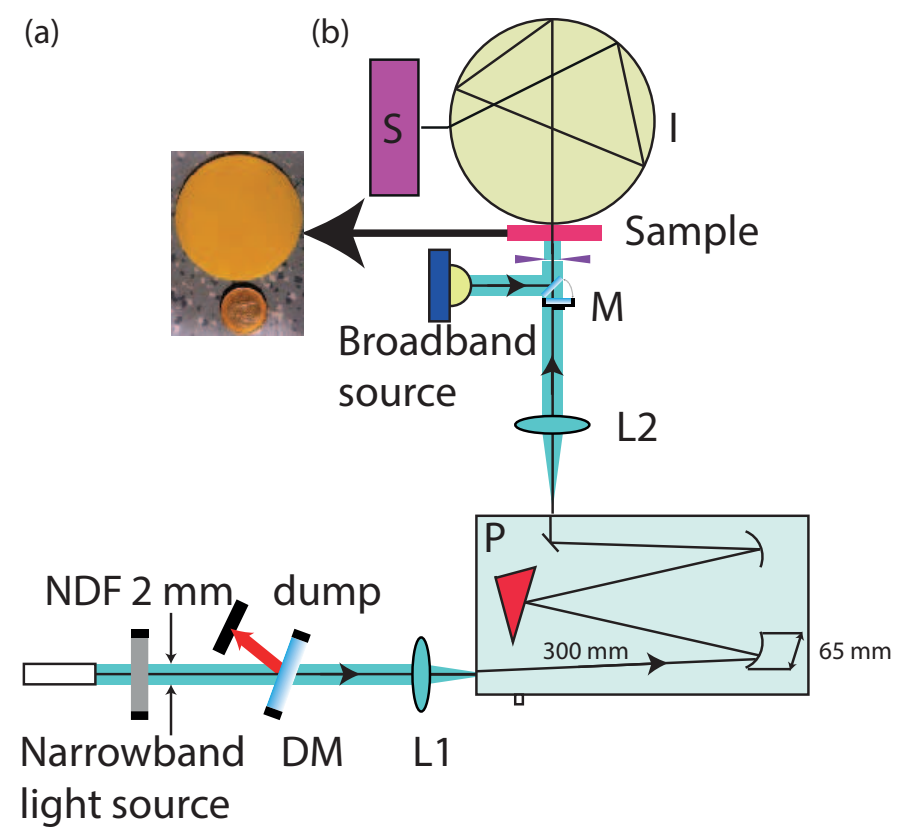

Figure 3.2: Narrowband measurement setup. (a) A polymer slab with a 4 wt\% YAG:Ce ${ }^{3+}$ compared to a 1 Euro cent. (b) Supercontinuum white light source Fianium, NDF: neutral density filter, DM: dichroic mirror, L1: achromatic doublet (AC080-010-A$\mathrm{ML}, \mathrm{f}=10 \mathrm{~mm}), \mathrm{L} 2$ : achromatic doublet $(\mathrm{f}=50 \mathrm{~mm}), \mathrm{M}$ : mirror, we: integrating sphere, $\mathrm{S}$ : spectrometer, P: prism spectrometer $\left(f_{\sharp}=4.6\right)$.

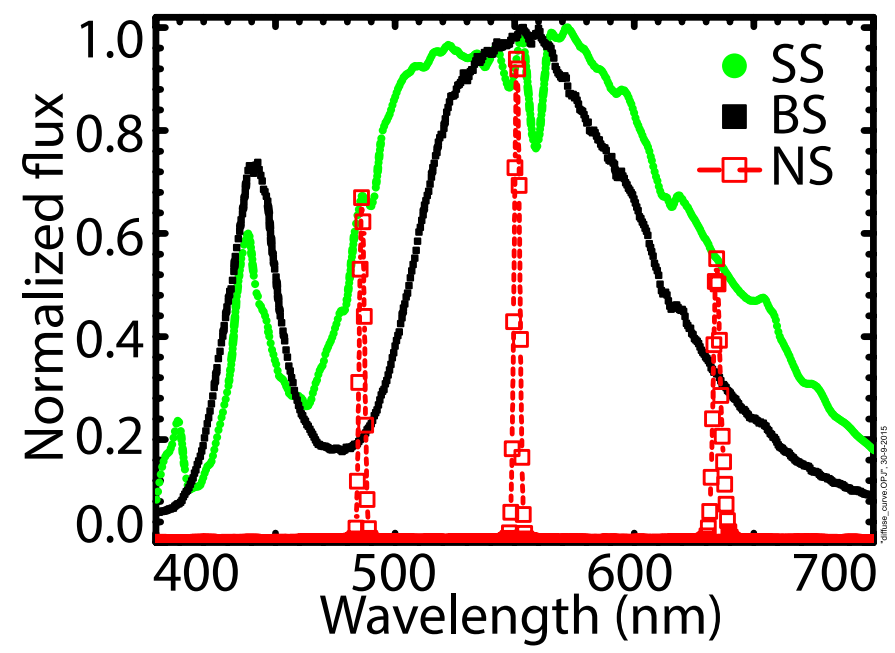

Figure 3.3: Normalized reference spectra of the light sources used in the experiment. Green circles - normalized reference spectrum of the supercontinuum source (SS) after being filtered by a neutral density filter and a dichroic mirror that removes the infrared part of the spectra. Black squares - normalized reference spectrum of a broadband source (BS) that was not filtered. Red squares - spectrally filtered narrowband light source (NS) normalized to the initial BS spectrum represent. We show narrowband spectra for three incident wavelengths $\lambda_{\mathrm{i}}=486,551,634 \mathrm{~nm}$. flux normalized to the SS. 


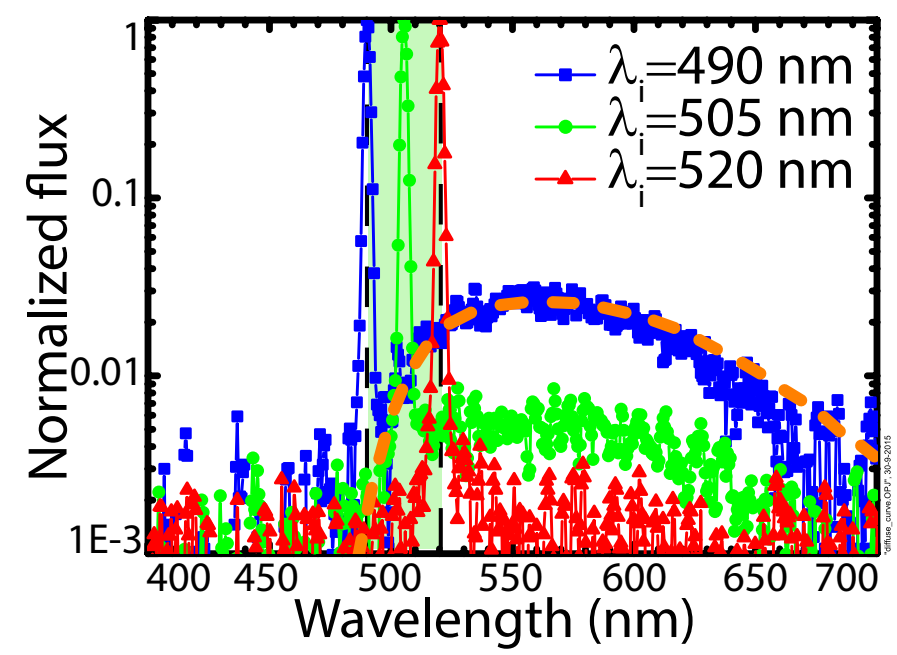

Figure 3.4: Flux profile of the signal that we measure in the range where emission and absorption overlap for three different pump wavelengths. The orange dashed line is the emission spectra from Fig. 3.1(a). The blue curve was normalized to 7791 counts and the green and the red curves to 15480 and 20297 counts respectively.

band and the broadband light source to check the consistency in the spectral ranges where both methods can be applied $\left(\lambda \leq \lambda_{\ell}\right.$ and $\left.\lambda \geq \lambda_{\mathrm{r}}\right)$. For the broadband light source, the scattered transmission is obtained by normalizing the measured spectrum $F_{\mathrm{T}}$ to a reference spectrum $F_{0}$ measured in the absence of a sample. For the narrowband light source, the scattered transmission is determined as the ratio of the transmitted flux $F_{\mathrm{T}}\left(\lambda_{\mathrm{i}}\right)$ and a reference flux $F_{0}\left(\lambda_{\mathrm{i}}\right)$ without the sample at the designated wavelength $\lambda_{\mathrm{i}}$ that is set with the monochromator. The scattered transmission is reproducible to within a few percent points on different measurements with different light sources.

\subsection{Results}

\subsubsection{Transmission measurements}

To measure the scattered transmission $T\left(\lambda_{\mathrm{i}}\right)$ of the polymer plates we tune the narrowband light source to an incident wavelength $\lambda_{\mathrm{i}}$ and measure the transmitted flux of the outgoing light. In Fig. 3.4 we show the normalized transmitted flux for three incident wavelengths $\lambda_{\mathrm{i}}$. For an incident wavelength $\lambda_{\mathrm{i}}=490 \mathrm{~nm}$, we see a pronounced peak with the maximum at $\lambda=490 \mathrm{~nm}$. This peak contains mostly elastically scattered photons because inelastically scattered photons are Stokes-shifted to longer wavelengths. Indeed between $500 \mathrm{~nm}$ and $650 \mathrm{~nm}$, the flux profile reveals a broad peak that represents the Stokes-shifted flux since the flux profile has a shape similar to that of the $\mathrm{YAG}: \mathrm{Ce}^{3+}$ in powder form (black dashed 
curve). Both emission spectra have a maximum at $557 \mathrm{~nm}$. For YAG:Ce ${ }^{3+}$ in powder form the flux is slightly higher at longer wavelengths $\lambda>600 \mathrm{~nm}$ than for the phosphor plate. The peak value of the Stokes-shifted light amounts to $3 \%$ of the transmitted flux at the incident wavelength $\lambda_{\mathrm{i}}=490 \mathrm{~nm}$. We calculate the amount of Stokes-shifted light $F_{\mathrm{em}}$ in the elastic peak at $\lambda_{\mathrm{i}}=490 \mathrm{~nm}$ by extrapolating the emission curve to this wavelength range. We find that $F_{\text {em }}$ amounts to less than $1 \%$ of the transmitted flux $F_{\text {tot }}$, and can be safely neglected. The total Stokes-shifted flux decreases drastically for longer incident wavelengths $\lambda_{\mathrm{i}}$ inside the overlap range, and can thus be ignored too. For incident light in the middle of the overlap range at $\lambda_{\mathrm{i}}=505 \mathrm{~nm}$, we see that the emitted flux is even weaker with a normalized flux $F_{\text {em }}$ less than $1 \%$ of $F_{\text {tot }}$. At the edge of the absorption band at the incoming wavelength $\lambda_{\mathrm{i}}=520 \mathrm{~nm}$ we observe an even lower Stokes-shifted flux $F_{\text {em }}$. The reason for this decrease is that the absorption cross section decreases drastically in the overlap range, and only very little light is being absorbed, and as a result is Stokesshifted. Throughout the overlap range, the contribution from the Stokes-shifted light $F_{\mathrm{em}}$ is less then $1 \%$ of $F_{\text {tot }}$ at the incident wavelength $\lambda_{\mathrm{i}}$. We thus conclude that the Stokesshifted flux contribution can be neglected throughout the overlap range. Therefore, we have distinguished the elastically scattered (or absorbed) fraction of light from the Stokes-shifted light. The amount of Stokes-shifted light strongly depends on the incident wavelength $\lambda_{\mathrm{i}}$. As a result, we can now measure scattered transmission at any desired wavelength.

We have scanned the incident wavelength $\lambda_{\mathrm{i}}$ through the wavelength range of interest (see Fig. 3.4). In Fig. 3.1(b) the scattered transmission $T(\lambda)$ for the slab with 4 wt\% of YAG: $\mathrm{Ce}^{3+}$ has been obtained from these scans (red squares). The total relative transmission $T_{\text {tot }}^{\mathrm{rel}}(\lambda)$ measured with the broadband source is also shown in Fig. 3.1 (b) for comparison (blue triangles). For short wavelengths, both transmission spectra coincide within a few percent. The spectrum measured with broadband light source reveals a deep trough with a minimum at $458 \mathrm{~nm}$. The trough matches well with the peak of the absorption band of YAG:Ce ${ }^{3+}$ in Fig. 3.1(a), and reveals that the phosphor absorbs a significant fraction of the light in this wavelength range. At wavelengths longer than $\lambda_{\mathrm{r}}=520 \mathrm{~nm}$ both transmission spectra are flat, but the spectrum measured with the broadband light source is 10 percent point larger than the spectrum measured with the narrowband light source. The spectrum measured with broadband light source contains a significant contribution of the Stokes-shifted light $F_{\text {em }}$ in this spectral range, as most of the emission occurs in this spectral range (see Fig. 3.1(a)). The narrowband spectrum, on the contrary, does not have this contribution. The difference between these two spectra at long wavelengths is equal to $T_{\mathrm{em}}\left(\lambda_{\mathrm{i}}\right)$, in Eq. (3.2). In the overlap region $\left(\lambda_{\ell}=490 \mathrm{~nm}<\lambda<\lambda_{\mathrm{r}}=520 \mathrm{~nm}\right)$ both 

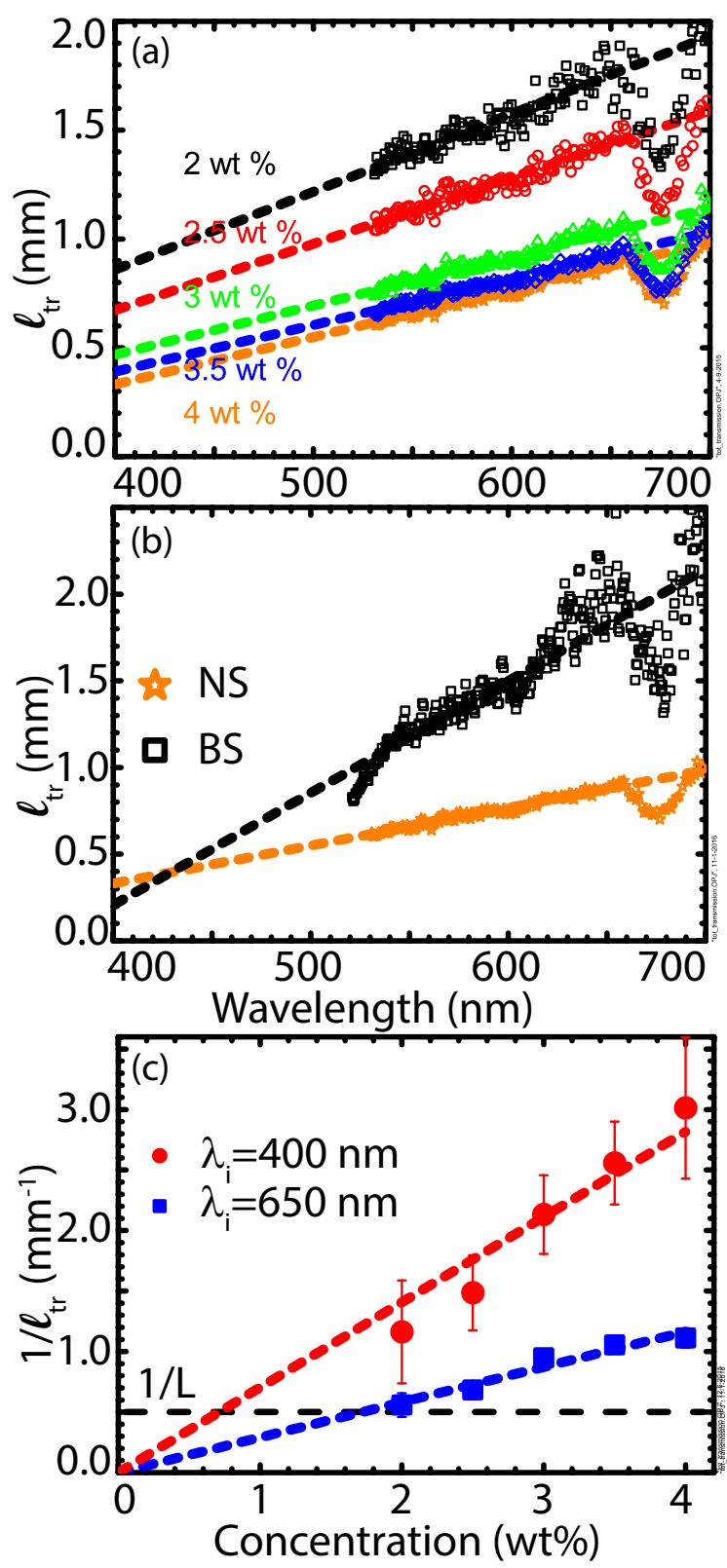

Figure 3.5: Transport mean free path. (a) Transport mean free path as a function of wavelength for different phosphor concentrations. Dashed lines represent linear fits to the measured data with parameters listed in table. 3.1. (b) Black squares indicate transport mean free path as a function of wavelength for broadband light source measurement. Orange stars indicate transport mean free path as a function of wavelength for narrowband light source measurement. Dashed lines represent linear fits to the measured data with parameters listed in table 3.1. Here concentration of the phosphor is taken to be $4 \mathrm{wt} \%$. (c) Inverse transport mean free path as a function of concentration for two different incoming wavelengths. Dashed lines are linear fits to the data.

spectra reveal a sharp rise. In this range, the Stokes-shifted light causes the total relative transmission $T_{\text {tot }}^{\text {rel }}$ to increasingly deviate from the scattered transmission. In Ref. 54 it 
was not possible to separate elastically scattered and Stokes-shifted light.

Finally, we filtered the broadband light source with a long pass filter at $\lambda=520 \mathrm{~nm}$, which ensures that the phosphor is not excited (we thus have zero emitted flux $T_{\mathrm{em}}\left(\lambda_{\mathrm{i}}\right)=0$ ). Hence, the measured total relative transmission $T_{\mathrm{tot}}^{\mathrm{rel}}$ equals the scattered transmission $T_{\mathrm{tot}}^{\mathrm{rel}}=$ $T$ in this spectral range. In Fig. 3.1(b) we compare the scattered transmission measured with the filtered broadband light source (green), and the scattered transmission measured with the narrowband light source (red). The scattered transmission measured with the narrowband light source agrees within a few percent points with the scattered transmission measured with the filtered broadband light source (see Fig. 3.1(b)) for $\lambda \geq \lambda_{\mathrm{r}}$. Concluding, we have for the first time extracted the scattered transmission $T\left(\lambda_{\mathrm{i}}\right)$ for a diffuser plate with phosphor in the whole visible range, including the previously inaccessible overlap range.

\subsubsection{Transport mean free path}

In the range of low absorption $(520 \mathrm{~nm}<\lambda<700 \mathrm{~nm})$ we have extracted the transport mean free path from the transmission data using Eq. 3.6 and plotted the result in Fig. 3.5(a). We see that the transport mean free path increases linearly with wavelength at constant phosphor concentration. In highly polydisperse non-absorbing media, a similar relationship between the transport mean free path and wavelength was found and successfully interpreted [53]. Therefore, we have fitted the transport mean free path with a line for every phosphor concentration. Parameters of the linear fits are listed in Table 3.1. We linearly extrapolate $\ell_{\text {tr }}$ to the absorption range $\lambda \leq \lambda_{\mathrm{r}}=520 \mathrm{~nm}$, and use the extrapolated values of the transport mean free path $\ell_{\operatorname{tr}}$ to obtain the absorption mean free path $\ell_{\mathrm{a}}$ in the range of strong absorption. We investigated a systematic error in the transmission when

the broadband light is used. We compare the apparent mean free path derived from $T_{\text {tot }}^{\text {rel }}$ with the true mean free path derived from $T$ (Fig. 3.5(b)). The apparent mean free path clearly differs from the true one. The difference is nearly $100 \%$ in the range between 500 and $700 \mathrm{~nm}$. Moreover, the slope is much steeper than for the true mean free path, as can be seen from Table 3.1 .

In the limit of low concentration, each scatterer can be treated independently. In this case $1 / \ell_{\mathrm{tr}}$ is proportional to the concentration of the scatterers $[70]$. Indeed in Fig. 3.5 (c), we see that the transport mean free path increases inversely proportional to the increasing phosphor concentration at a fixed wavelength. We observe that with increasing wavelength, the scattering cross section increases similarly to what was obtained earlier [54]. 


\begin{tabular}{|l|lll|lll|}
\hline $\mathrm{C}(\mathrm{wt} \%)$ & $\mathrm{a}$ & & & $\mathrm{b}$ & & \\
\hline 2.0 & -0.57 & \pm & 0.07 & 0.00358 & \pm & 0.00001 \\
\hline 2.5 & -0.55 & \pm & 0.05 & 0.00305 & \pm & 0.00008 \\
\hline 3.0 & -0.43 & \pm & 0.03 & 0.00225 & \pm & 0.00055 \\
\hline 3.5 & -0.47 & \pm & 0.02 & 0.00214 & \pm & 0.00038 \\
\hline 4.0 & -0.54 & \pm & 0.02 & 0.00218 & \pm & 0.00038 \\
\hline $4.0(\mathrm{BS})$ & -2.38 & \pm & 0.14 & 0.00640 & \pm & 0.00023 \\
\hline
\end{tabular}

Table 3.1: Parameters of the linear models of the transport mean free path versus wavelength: $\ell_{\mathrm{tr}}=a+b \lambda$. The parameters $a$ and $b$ depend on the phosphor concentration $\rho$ and are shown with their standard errors. 4 (BS) - linear fit for data measured with the broadband light source.

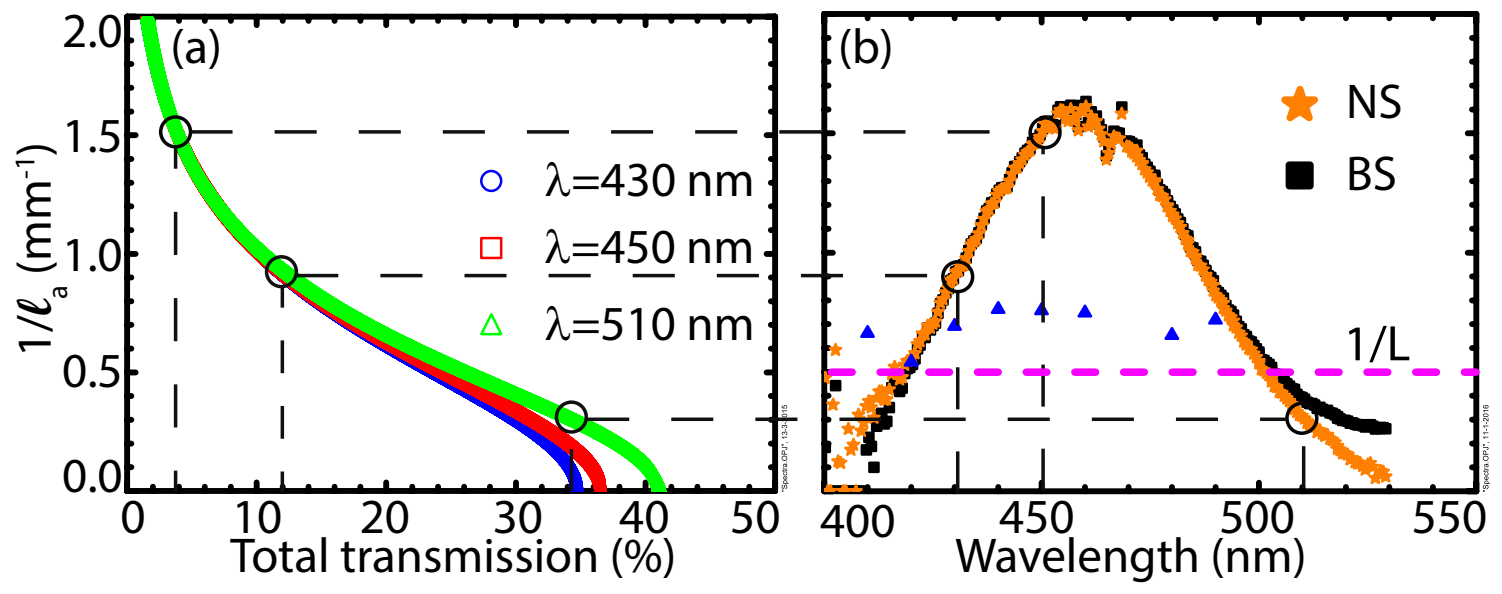

Figure 3.6: Determination of the absorption mean free path. (a) Look-up tables presented in a form of plots for Eq. 3.3 for three different wavelengths, and $4 \mathrm{wt} \%$ concentration of the phosphor. ( $\ell_{\mathrm{tr}}=0.40 ; 0.44 ; 0.57 \mathrm{~mm}$ respectively) (b) Orange stars show inverse absorption mean free path extracted from the look-up tables for the plate with 4 wt\% phosphor concentration (narrowband light source). Black squares show inverse absorption mean free path extracted using broadband light source for the same plate. Black circles and black dashed lines indicate the process of mapping $\mu_{\mathrm{a}}$ from the look-up table to the Fig. 3.6(b). Blue triangles indicate the inverse absorption length measured in Ref. [54]. The dashed purple line indicates the inverse thickness of the sample.

\subsubsection{Absorption mean free path}

By using the transport mean free path $\ell_{\text {tr }}$ extrapolated to the wavelength range between 400 and $530 \mathrm{~nm}$ where the phosphor absorbs light, we now derive the absorption mean free path $\ell_{\mathrm{a}}$ from the measured scattered transmission (see Fig. 3.1(c)). Since we do not have an analytic inverse function of Eq. (3.3), we have solved the inversion numerically and made 
look-up tables for each phosphor concentration and at each wavelength. Fig. 3.6(a) shows three inverted curves of $\mu_{\mathrm{a}}$ versus scattered transmission for three different wavelengths $(\lambda=430,450,510 \mathrm{~nm})$ at a phosphor concentration $\rho=4 \mathrm{wt} \%$. We plot absorption coefficient $\mu_{\mathrm{a}}$ as a function of transmission $T$ because $\ell_{\mathrm{a}}$ tends to infinity for vanishing absorption. Fig. 3.6 (a) shows that $\mu_{\mathrm{a}}$ (and thus the absorption) increase. In the limit of strong absorption, all scattered transmission curves tend to zero. In the limit of vanishing $\mu_{\mathrm{a}}$, the scattered transmission equals the scattered transmission from Eq. 3.6 that decrease with decreasing wavelength (Fig. 3.6(a)). The vertical dashed lines indicate the measured scattered transmission, and the intersections with the curves yield the corresponding $\mu_{\mathrm{a}}$ for each wavelength at this phosphor concentration.

Fig. 3.6(b) shows the extracted absorption profile $\mu_{\mathrm{a}}$ for the polymer plate with the highest phosphor concentration $\rho=4 \mathrm{wt} \%$ studied here. The FWHM of this curve is $64.5 \mathrm{~nm}$. The absorption mean free path $\ell_{\mathrm{a}}$ is shorter than the thickness of the sample $d$ between 418 and $501 \mathrm{~nm}$. This means that incident light is effectively absorbed in the volume of the sample, and the density of the phosphor is optimized for use in a whitelight LED. At the edges of the absorption range at 400 and $530 \mathrm{~nm} \mu_{\mathrm{a}}$ tends to zero, as expected from the known absorption (Fig. 3.1(a)). We note that our present $\mu_{\mathrm{a}}$ values differ from previous results obtained on the same samples [54] (see Fig. 3.6.(b)). The absorption mean free path varies significantly with a wavelength in our case. We notably attribute the difference to the use of incorrect use of diffusion equation in Ref. 54. The spectral shape obtained at present is in much better agreement with the phosphor absorption spectra than previously.

In Fig. 3.6.(b), we also show the apparent inverse absorption length that is derived from the total relative transmission $T_{\text {tot }}^{\text {rel }}(B S)$. In a significant part of the absorption range $(420<\lambda<500 \mathrm{~nm})$, there is little difference in the exact absorption length derived from the scattered transmission $T$ (NS). The reason for the small difference is apparent from Fig. 3.6(a). In Fig. 3.6(a) the absorption length differs little with the scattered transmission $T$ for $T<20 \%$ independent of the transport mean free path. At low absorption $(\lambda<500 \mathrm{~nm}$ and $\lambda>520 \mathrm{~nm}$ ) the difference between the apparent and the actual absorption mean free path becomes apparent.

In Fig. 3.7(a) we have plotted $\mu_{\mathrm{a}}(\lambda)$ for three wavelength $\lambda=430,460$ and $500 \mathrm{~nm}$ as a function of phosphor concentration. We see that $\mu_{\mathrm{a}}(\lambda)$ increases linearly with increasing phosphor concentration, which agrees with the assumption that each absorber is an independent absorber. Figure 3.7(a) shows that the steepest slope appears at the wavelength $\lambda$ $=460 \mathrm{~nm}$, which corresponds to the peak of the absorption curve. The maximum absorp- 

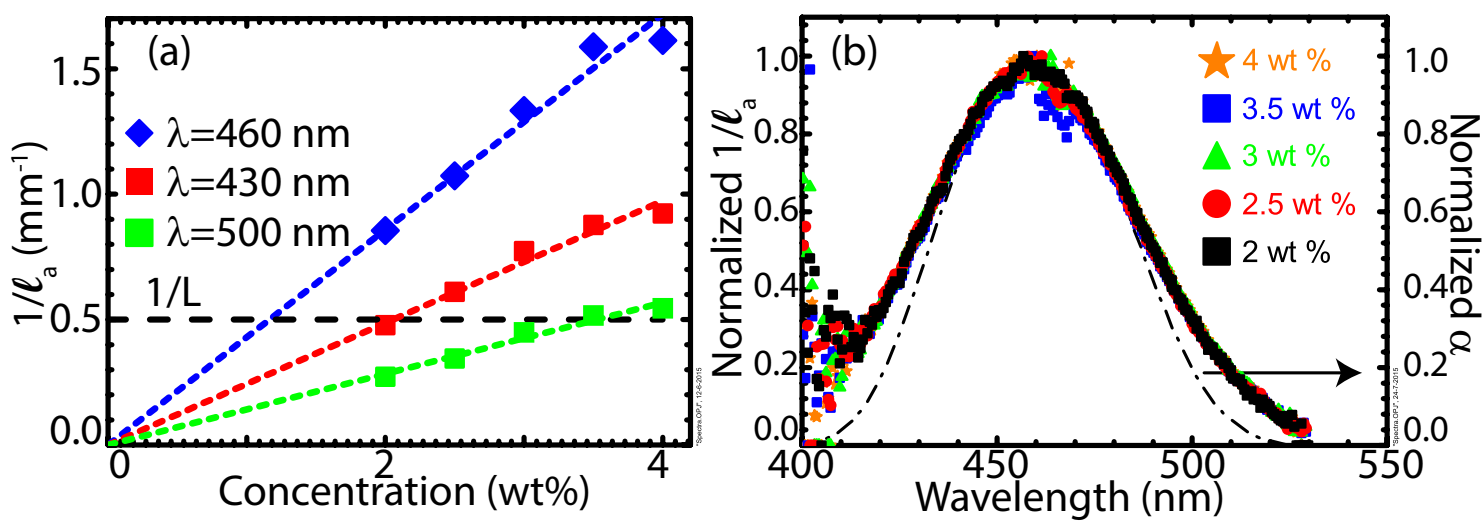

Figure 3.7: Absorption mean free path. (a) $\mu_{\mathrm{a}}$ as a function of concentration is shown for the three different wavelength. (b) Line shapes of the absorption coefficient are shown for the phosphor in powder (dot-dashed black line) and in polymer plates with increasing phosphor concentrations.

tion cross section is $\sigma_{\mathrm{a}}=30 \mu \mathrm{m}^{2}$, which agrees reasonably well with the typically measured absorption cross section for YAG: $\mathrm{Ce}^{3+}$ of the order of $10 \mu \mathrm{m}^{2}$ (Ref. [17,71,75]).

Figure 3.7(b) shows the extracted normalized absorption spectrum of $\mu_{\mathrm{a}}$ for the polymer plates with different phosphor concentrations. All absorption curves are normalized to their maximum, and they coincide with each other within a few percent points. The inverse absorption mean free path $\mu_{\mathrm{a}}$ scales linearly with the phosphor concentration for all wavelengths. All absorption curves tend to zero outside $400<\lambda<530 \mathrm{~nm}$.

In Fig. 3.7 (b) we also compare the shape of the absorption curve of the phosphor in powder form that was used to manufacture the samples to $\mu_{\mathrm{a}}$ curves. The black dot-dashed line shows absorption spectra of the $\mathrm{YAG}: \mathrm{Ce}^{3+}$ in powder. These two sets of data were normalized to their maxima, so the positions of maximums of these two graphs coincide. The $\mu_{\mathrm{a}}$ spectrum appear to have broader tails compared to the YAG: $\mathrm{Ce}^{3+}$ in powder form. The absorption spectrum of YAG:Ce ${ }^{3+}$ in powder has an FWHM $=54 \mathrm{~nm}$ that is $10 \mathrm{~nm}$ smaller than the FWHM of the measured absorption spectra. One possible reason for the difference is that light is multiply internally reflected in the YAG: $\mathrm{Ce}^{3+}$ particles [17], so particles cannot be treated as independent scatterers.

Finally, let us place our approach in the context of previous work: Vos et al. reported the transport properties measurements in $\mathrm{TiO}_{2}$ scattering plates using a broadband light source [53]. They showed that the transport mean free path $\ell_{\operatorname{tr}}$ linearly depends on the wavelength in the visible wavelength range. This method cannot be applied to phosphor doped plates, as these plates have ranges with strong absorption, emission, and an overlapping range. Leung et al. reported the transport properties measurements in phosphor 
doped slabs using a filtered broadband light source, where the linear dependence of $\ell_{\operatorname{tr}}$ was exploited to calculate $\ell_{\mathrm{a}}$ [54]. Unfortunately, however, the approximation used to analyze scattered transmission $T\left(\lambda_{\mathrm{i}}\right)$ from Ref. [54] was used outside its range of validity. Secondly, the described method is limited to the region of strong absorption or emission but is not valid in the overlap region.

\subsection{Summary and outlook}

In this chapter, we have been able to separate light of the same wavelength yet originated from different physical processes occurring in the polymer plates of solid-state lighting units. This became possible by introducing narrowband light source in the setup. The separate measurement of elastically scattered and Stokes-shifted light allows us to extract the transport and absorption mean free path of the given polymer plates, which are the essential parameters required for modeling and predicting the color spectra of solid-state lighting devices. The optimal parameters of the solid-state lighting units can be directly extracted from the measured absorption curves. We vary the thickness of the plate $d$ or the phosphor concentration depending on the desired level of pump absorption using absorption curve in Fig. 3.6(b).

We have developed a new technique to measure the light transport of white-light LED plates in the visible range based on narrowband illumination and spectrally sensitive detection. We compare the data obtained with the new technique to the data measured with the broadband light source. The two sets of data coincide in the range without absorption $\lambda \geq \lambda_{\mathrm{r}}$, as well as in the range with strong absorption $\lambda \leq \lambda_{\ell}$. We extracted the scattered transmission in the overlap range that was previously inaccessible.

We used diffusion theory to extract transport $\ell_{\operatorname{tr}}$ and absorption $\ell_{\mathrm{a}}$ mean free paths from these data in the previously inaccessible range. The shape of the absorption coefficient measured for the YAG: $\mathrm{Ce}^{3+}$ powder, and YAG: $\mathrm{Ce}^{3+}$ powder in a polymer matrix have similar trends, although for the polymer plates the curve is broader than for the YAG:Ce ${ }^{3+}$ powder. Both $\mu_{\mathrm{a}}$ and $1 / \ell_{\mathrm{tr}}$ are proportional to the concentration of phosphor, which reveals that elastic and inelastic processes do not influence each other.

By exploiting narrowband light source and interpreting the resulting scattered transmission by diffusion theory, we extracted for the first time light transport parameters for white LEDs in the whole visible wavelength range. However, diffusion theory only gives an analytical solution for simple sample geometries, such as a slab, a sphere, or a semiinfinite medium. Therefore, to efficiently model a real white LED with a complex geometry 
we must, in the end, supplement an ab initio theory with a numerical method. An important class of numerical methods is the Monte Carlo methods, wherein the case of light in arbitrary geometries the ray tracing techniques are a major workhorse [15-18]. Future work should, therefore, focus on the question of how to combine analytical methods with numerical ones. 


\section{Chapter 4}

\section{Accurate determination of transport parameters in strongly absorbing and scattering materials}

\subsection{Introduction}

Currently, light propagation through scattering and strongly absorbing optical systems, like white LEDs, is described using various numerical methods such as Monte Carlo simulations and ray tracing $[15,18,76]$. While the computational methods are incorporate the detailed geometry of the problem at hand, they also have several substantial limitations. There is a trade-off between the obtained precision and the speed of numerical approaches that ultimately compromises the design of white LEDs, since accuracy increases with the number of numerical samples taken, thereby naturally decreasing speed. Moreover, the accuracy of numerical methods is not always beyond doubt, as a posteriori readjustments of the transport parameters have been reported $[17,18$. Numerical methods do not possess predictive power for different LEDs than the one calculated, as opposed to analytical methods. Hence for every new situation (e.g., new phosphor, new blue LED, etc.) new simulations must be performed. Therefore, it is timely to search for analytic approaches that have predictive power (even outside the immediately studied parameter domain), and that are accurate and fast.

In the optically thin samples $d / \ell_{\operatorname{tr}}<<1$ (see Fig. 4.1), the transport of light is analytically described by the venerable Lambert-Beer (or Beer-Lambert-Bouguer) law. In scattering systems such as white LEDs, however, the condition $d / \ell_{\operatorname{tr}} \approx 1$ holds [53, 54, hence light is scattered multiple times and diffused 1 . Moreover, we will see that in certain

\footnotetext{
${ }^{1}$ To be sure, in a scattering system the degree of scattering is given by $d / \ell_{s}$ rather than $d / \ell_{t r}$. Since in
} 


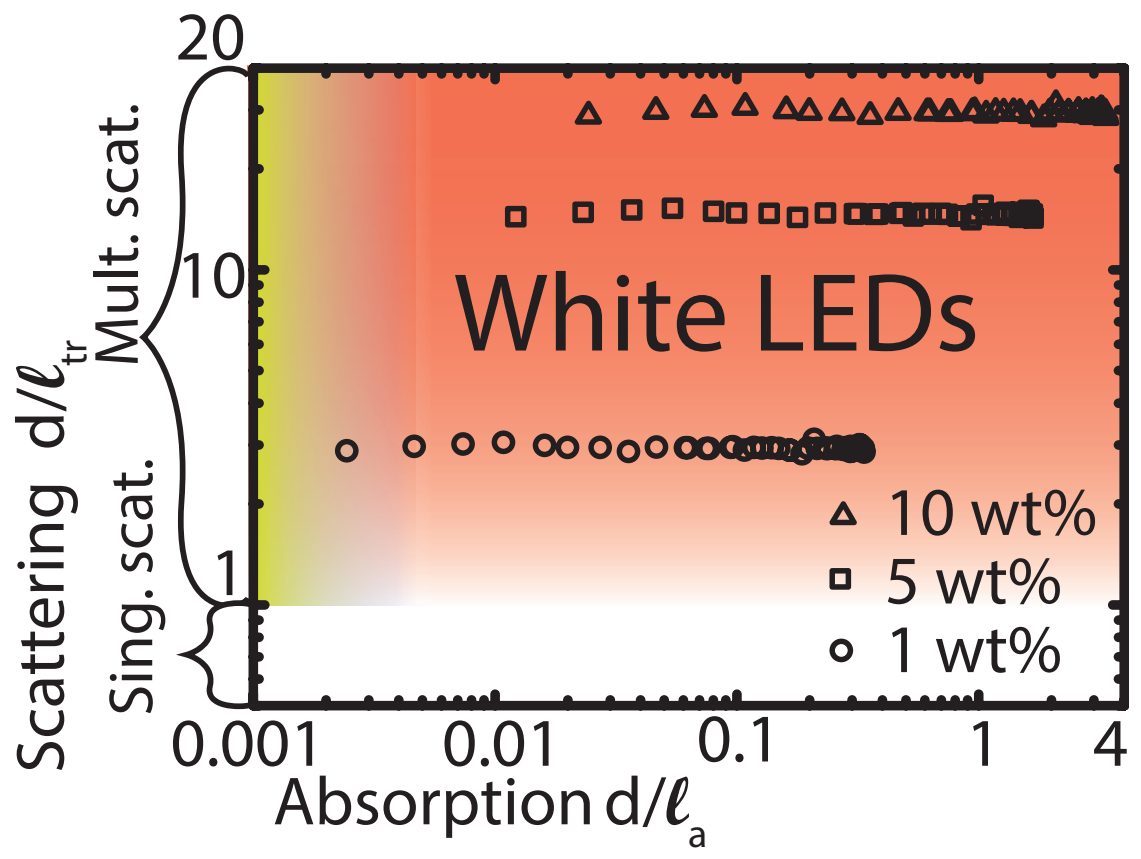

Figure 4.1: Transport parameters in the plane spanned by absorption and diffusion. The absorption on the abscissa is gauged by the ratio of sample thickness $d$ and absorption mean free path $\ell_{\mathrm{a}}$ and the diffusion strength on the ordinate by the ratio of sample thickness $d$ and transport mean free path $\ell_{\text {tr }}$. The shaded yellow range indicates the area where the diffusion theory performs well. The red range represents the transport parameter range accessed in the current manuscript. Symbols represent presently measured absorption and scattering as a function of wavelength for phosphor diffuser plates with particle concentrations $1-10 \mathrm{wt} \%$. The gradient on the figure shows artistic representation of the diffusion theory applicability.

common circumstances, light is also strongly absorbed $d / \ell_{\mathrm{a}} \geq 1$. A widely known theory of transport of light is the radiative transfer equation [42. While the full radiative transfer equation requires numerical solutions (for example Monte Carlo simulations), there are several relevant cases where analytical solutions exist. The first order approximation to radiative transfer equation is the diffusion equation (see Ch. 22 [23, 25, 26, 77, 78. Solutions to the diffusion equation work surprisingly well even in regimes where they are not supposed to function [23] such as optically thin samples [24,53. Regarding absorption, however, the validity of the diffusion theory is limited to weakly absorbing systems with $d / \ell_{\mathrm{a}} \ll 1$, as indicated in Fig. 4.1 and Ch. 6] [43, 77]. In white LEDs, however, the absorption mean free path at maximum absorption is at least four times smaller than the sample thickness

the vast majority of scattering systems the transport mean free paths exceeds the scattering mean free path $\left(\ell_{t r} \geq \ell_{s}\right)$ due to predominantly forward scattering, we consider our characterization to be on the safe side. 
$d / \ell_{\mathrm{a}} \geq 4$, see Fig. 4.1. Since in this regime the diffusion theory fails to describe the light propagation, other analytical approaches are required to accurately determine transport parameters of scattering materials with absorption, and to offer fast design tools for white LEDs.

In this chapter, we describe an analytical, accurate, and fast approach to determine the transport parameters in samples that are used in white LEDs. The determination of the transport parameters involves the measurement of the total transmission, the scattered reflection, and the ballistic transmission for a set of diffuser plates as a function of the concentration of the phosphor particles. The total transmission and the scattered reflection data are analyzed using the P3 approximation to the radiative transfer equation (RTE) [33]. In optically thin scattering samples where the contribution of the ballistic light dominates, the data were analyzed using the Lambert-Beer law. To verify our approach, we performed Monte Carlo simulations and found excellent agreement between the numerically obtained transport parameters and analytically obtained transport parameters. The demonstrated approach offers a fast and accurate determination of the transport parameters and possesses predictive power beyond the parameter range studied. The precision of the extracted transport parameters is only limited by the errors of the experimental data. Besides, the proposed approach of measuring the total transmission $T$ and the scattered reflection $R$ allows us to decouple the scattering and the absorption in a straightforward experimental way. Once transport parameters are known, they can be used as input parameters in the ray tracing Monte Carlo calculations for white LED design which requires complex geometries that are not captured by the analytical approach [79. We show that the parameter extraction is also applicable within the domain of validity of the diffusion theory (see Ch. 6). Hence, the proposed parameter extraction approach pertains also to the propagation of light in other relevant application fields, such as oceans and clouds 80 84, pharmaceutical products 38 40, and in noninvasive diagnostic imaging of living tissues 32 37.

\subsection{Model}

In this chapter the diffuser plate with phosphor particles is modeled as a slab of thickness $\mathrm{d}$ that extends from $z=0$ to $z=d$, as shown in Fig. 4.2. Incident plane waves at wavelength $\lambda$ with flux $F_{\mathrm{i}}(\lambda, z=0, \rho)=F_{\mathrm{i}}(\lambda, 0, \rho)$ illuminate the slab, where $\rho$ is the particle density. The incident light is scattered multiple times and possibly absorbed inside the slab. We characterize the light scattering and absorption in the slab by measuring the total transmission $T$, the scattered reflection $R$, and the ballistic transmission $T_{\mathrm{b}}$ that are 


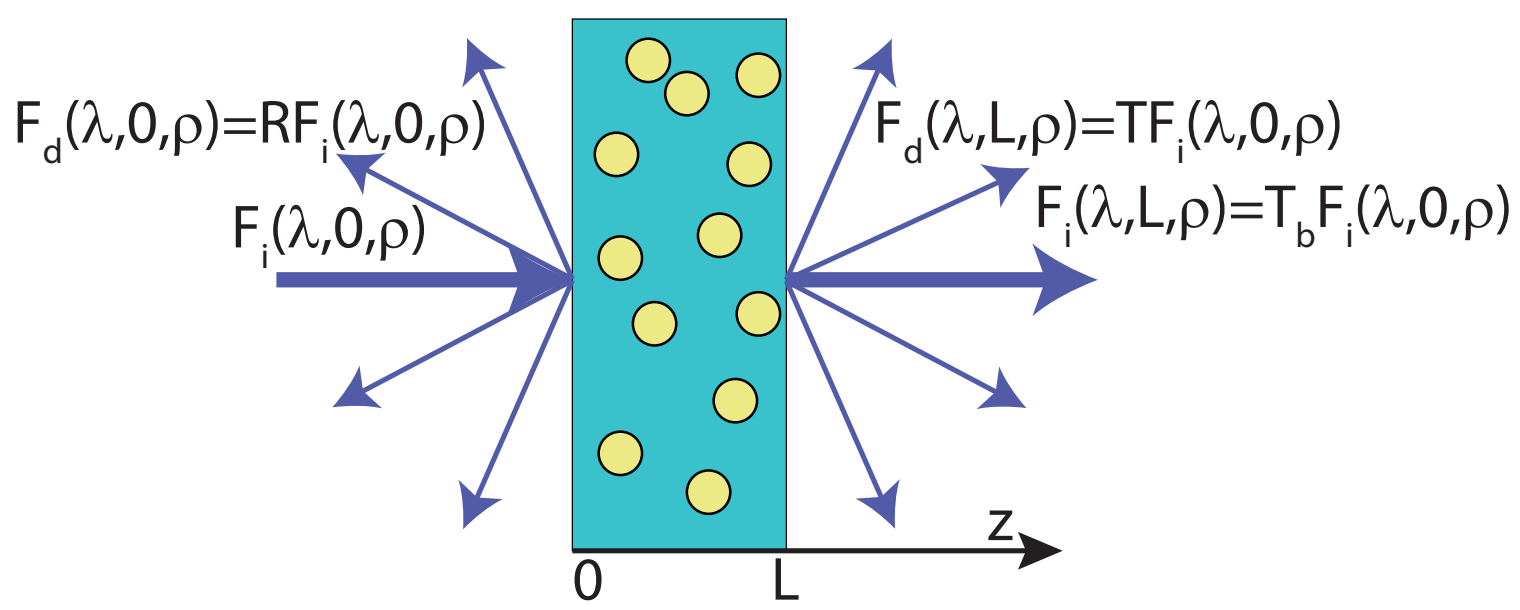

Figure 4.2: Scheme of light incident on and exiting from a slab. Plane waves with flux $F_{\mathrm{i}}(\lambda, 0, \rho)$ are incident in the $z$-direction on a slab of scattering material that contains phosphor particles (represented by yellow circles). $F_{\mathrm{d}}(\lambda, 0, \rho)$ is the diffuse reflected flux, $F_{\mathrm{d}}(\lambda, \mathrm{d}, \rho)$ is the diffuse transmitted flux, and $F_{\mathrm{i}}(\lambda, \mathrm{d}, \rho)$ is the transmitted ballistic flux. $T$ and $R$ are the total transmission and the scattered reflection, respectively, and $T_{\mathrm{b}}$ is the ballistic transmission.

defined as

$$
T(\lambda, d, \rho) \equiv \frac{F_{\mathrm{T}}(\lambda)}{F_{0}(\lambda)}=\frac{F_{\mathrm{d}}(\lambda, \mathrm{d}, \rho)+F_{\mathrm{i}}(\lambda, \mathrm{d}, \rho)}{F_{\mathrm{i}}(\lambda, 0, \rho)},
$$

and

$$
R(\lambda, 0, \rho) \equiv \frac{F_{\mathrm{d}}(\lambda, 0, \rho)}{F_{\mathrm{i}}(\lambda, 0, \rho)}
$$

and

$$
T_{\mathrm{b}}(\lambda, d) \equiv \frac{F_{\mathrm{i}}(\lambda, \mathrm{d}, \rho)}{F_{\mathrm{i}}(\lambda, 0, \rho)}
$$

where $F_{\mathrm{T}}(\lambda)$ is the elastic transmitted flux, $F_{\mathrm{d}}(\lambda, 0, \rho)$ and $F_{\mathrm{d}}(\lambda, \mathrm{d}, \rho)$ are the diffused flux integrated over all outgoing angles at the positions $z=0$ and $z=\mathrm{d}$, respectively, and $F_{\mathrm{i}}(\lambda, \mathrm{d}, \rho)$ is the ballistic transmitted flux. The measured total transmission $\mathrm{T}$, the scattered reflection $\mathrm{R}$, and the ballistic transmission $T_{\mathrm{b}}$ explicitly depend on the experimentally available parameters: the wavelength $\lambda$, the thickness of the sample $d$, and the density $\rho$ of the phosphor particles. We will use measured total transmission $T$, scattered reflection $R$, and ballistic transmission $T_{\mathrm{b}}$ to extract average single particle properties: the scattering cross section $\sigma_{\mathrm{s}}$, the absorption cross section $\sigma_{\mathrm{a}}$, and the anisotropy factor $\mu$ [41].

To extract the average single particle properties $\left(\sigma_{\mathrm{s}}, \sigma_{\mathrm{a}}, \mu\right)$ we exploit an analytical model to compute the total transmission $T^{\mathrm{m}}$, the scattered reflection $R^{\mathrm{m}}$ and the ballistic transmission $T_{\mathrm{b}}^{\mathrm{m}}$ that not only explicitly depend on the wavelength $\lambda$, the thickness of the sample $d$, and the density $\rho$ of the phosphor particles, but also on single particle properties 
$\left(\sigma_{\mathrm{s}}, \sigma_{\mathrm{a}}, \mu\right)$. By equating $\left(T^{\mathrm{m}}, R^{\mathrm{m}}, T_{\mathrm{b}}^{\mathrm{m}}\right)$ to the experimentally measured $\left(T, R, T_{\mathrm{b}}\right)$ we compute the transport parameters $\left(\sigma_{\mathrm{s}}, \sigma_{\mathrm{a}}, \mu\right)$. As the analytical model we chose to employ the P3 approximation to the radiative transfer equation $33,35,37,45,51,85$

$$
T^{\mathrm{m}}\left(\lambda, d, \rho ; \sigma_{\mathrm{s}}, \sigma_{\mathrm{a}}, \mu\right) \equiv \frac{F_{\mathrm{d}}\left(\lambda, d, \rho ; \sigma_{\mathrm{s}}, \sigma_{\mathrm{a}}, \mu\right)+F_{\mathrm{i}}\left(\lambda, d, \rho ; \sigma_{\mathrm{s}}, \sigma_{\mathrm{a}}, \mu\right)}{F_{\mathrm{i}}\left(\lambda, d, \rho ; \sigma_{\mathrm{s}}, \sigma_{\mathrm{a}}, \mu\right)},
$$

and

$$
R^{\mathrm{m}}\left(\lambda, d, \rho ; \sigma_{\mathrm{s}}, \sigma_{\mathrm{a}}, \mu\right) \equiv \frac{F_{\mathrm{d}}\left(\lambda, d, \rho ; \sigma_{\mathrm{s}}, \sigma_{\mathrm{a}}, \mu\right)}{F_{\mathrm{i}}\left(\lambda, d, \rho ; \sigma_{\mathrm{s}}, \sigma_{\mathrm{a}}, \mu\right)}
$$

with

$$
F_{\mathrm{d}}\left(\lambda, z, \rho ; \sigma_{\mathrm{s}}, \sigma_{\mathrm{a}}, \mu\right)=\sum_{i=1}^{4} B_{1 \mathrm{i}} \exp \left(\mu_{i} z\right)+G_{1} \exp \left(-\rho \sigma_{\mathrm{t}} z\right)
$$

where $\sigma_{\mathrm{t}}=\sigma_{\mathrm{s}}+\sigma_{\mathrm{a}}$ is the sum of the scattering cross section $\sigma_{\mathrm{s}}$ and the absorption cross section $\sigma_{\mathrm{a}}$, and $B_{\mathrm{mi}}$ when $m=1, G_{\mathrm{m}}$ when $m=1$ and $\mu_{\mathrm{i}}$ are functions described in the Ch. 2, $\rho$ is the particle concentration in the samples, and $F_{\mathrm{i}}\left(\lambda, d, \rho ; \sigma_{\mathrm{s}}, \sigma_{\mathrm{a}}, \mu\right)$ is the incident light flux and $I_{\mathrm{d}}\left(\lambda, z, \rho ; \sigma_{\mathrm{s}}, \sigma_{\mathrm{a}}, \mu\right)$ the flux of the light at position z.

The ballistic flux that is transmitted through the sample is described by the LambertBeer law

$$
T_{\mathrm{b}}^{\mathrm{m}}\left(\lambda, d, \rho ; \sigma_{\mathrm{a}}, \sigma_{\mathrm{s}}\right) \equiv \frac{F_{\mathrm{d}}\left(\lambda, z, \rho ; \sigma_{\mathrm{s}}, \sigma_{\mathrm{a}}, \mu\right) e^{-\left(\rho \sigma_{\mathrm{t}} d\right)}}{F_{\mathrm{d}}\left(\lambda, z, \rho ; \sigma_{\mathrm{s}}, \sigma_{\mathrm{a}}, \mu\right)}=\exp \left(-\rho \sigma_{\mathrm{t}} d\right)
$$

Knowledge of the average single particle scattering properties $\left(\sigma_{\mathrm{s}}, \sigma_{\mathrm{a}}, \mu\right)$ at any wavelength $\lambda$ allows us to predict the light transport properties for any scattering and absorbing sample for any particle density $\rho$ and sample thickness $d$ outside the immediately studied density and thickness range. Therefore, it is convenient to express the single-particle properties into the scattering mean free path $\ell_{\mathrm{s}}$ the transport mean free path $\ell_{\operatorname{tr}}$ and the absorption mean free path $\ell_{\mathrm{a}}$ from the following relationships $[54,86$

$$
\begin{gathered}
\ell_{\mathrm{s}}=\frac{1}{\rho \sigma_{\mathrm{s}}}, \\
\ell_{\mathrm{a}}=\frac{1}{\rho \sigma_{\mathrm{a}}}, \\
\frac{1}{\ell_{\mathrm{tr}}}=\frac{(1-\mu)}{\ell_{\mathrm{s}}}+\frac{1}{\ell_{\mathrm{a}}},
\end{gathered}
$$

provided the underlying assumptions of the independent scatterer approach 23 namely negligible multiparticle effects, negligible re-absorption, and negligible absorption of the 
medium in which particles are embedded remain fulfilled.

\subsection{Experimental details}

The samples studied in the experiments are $d=1.98 \pm 0.02 \mathrm{~mm}$ thick silicone plates (polydimethylsiloxane, PDMS, refractive index $n=1.4[87]$ ) that are doped with YAG:Ce ${ }^{3+}$ phosphor particles in a range of particle concentration $\rho$ ranging from 1 to 10 weight percent phosphor. The absorption spectrum of the phosphor particles has a peak absorption wavelength $\lambda=458 \mathrm{~nm}$, and a broad linewidth (full width half maximum, FWHM) of $\Delta \lambda_{\mathrm{a}}=55 \mathrm{~nm}$, see Ch. 3. Based on the absorption spectrum we choose the wavelength $\lambda_{\mathrm{r}}=520 \mathrm{~nm}$ to distinguish the absorbing range $(\lambda=400 \mathrm{~nm}$ to $520 \mathrm{~nm})$ from the nonabsorbing range $(\lambda=520 \mathrm{~nm}$ to $700 \mathrm{~nm})$, as in Ch. 3.

Figure 4.3 shows a schematic of the experimental setup used to measure the total transmission $T$, the total reflectance $\mathrm{R}$ and the ballistic transmission $T_{\mathrm{b}}$. The sample is illuminated with a tunable narrowband light source in the visible wavelength range $420-700 \mathrm{~nm}$. To this end, the beam of a supercontinuum white-light source (Fianium WL-SC-UV-3) is spectrally filtered to an adjustable bandwidth $\Delta \lambda<2.4 \mathrm{~nm}$ using a prism monochromator (Carl-Leiss Berlin-Steglitz). Unwanted emission of the supercontinuum source in the infrared $\lambda_{\mathrm{i}}>700 \mathrm{~nm}$ is blocked with a neutral density filter (NENIR30A) and a dichroic mirror (DMSP805), see Fig. 4.3(a).

The incident beam illuminates the sample at normal incidence. The scattered flux is collected using either an integrating sphere or a ballistic detector. The integrating sphere (Opsira uku-240) has three entrance ports, each with a $20 \mathrm{~mm}$ diameter. Each port can be selectively closed with a baffle that has the same diffusive inner coating as the remainder of the integrating sphere. The entrance port of the integrating sphere is sufficiently large to collect scattered light that emanates at all angles from the strongest scattering sample. The flux of the outgoing light entering the integrating sphere is analyzed with a fiber-to-chip spectrometer (AvaSpec-USB2-ULS2048L) with a spectral resolution $\Delta \lambda_{\mathrm{S}}=2.4 \mathrm{~nm}$.

To measure the diffuse reflectance $R$, the ports 1 and 3 of the integrating sphere are used, see Fig. 4.3(b). The sample is attached to port 3 with the incident light flux $F_{\mathrm{i}}(\lambda, 0, \rho)$ entering the sphere through port 1 . The diffuse reflected light flux $F_{\mathrm{d}}(\lambda, 0, \rho)$ is collected with the fiber spectrometer. The reference flux $F_{\mathrm{i}}(\lambda, 0, \rho)$ separately enters the sphere under an angle through port 1 , in such way that the beam is incident on the surface of the sphere but not onto the sample.

To measure the total transmission, the ports 1 and 2 are used, as shown in Fig. 4.3(c). 


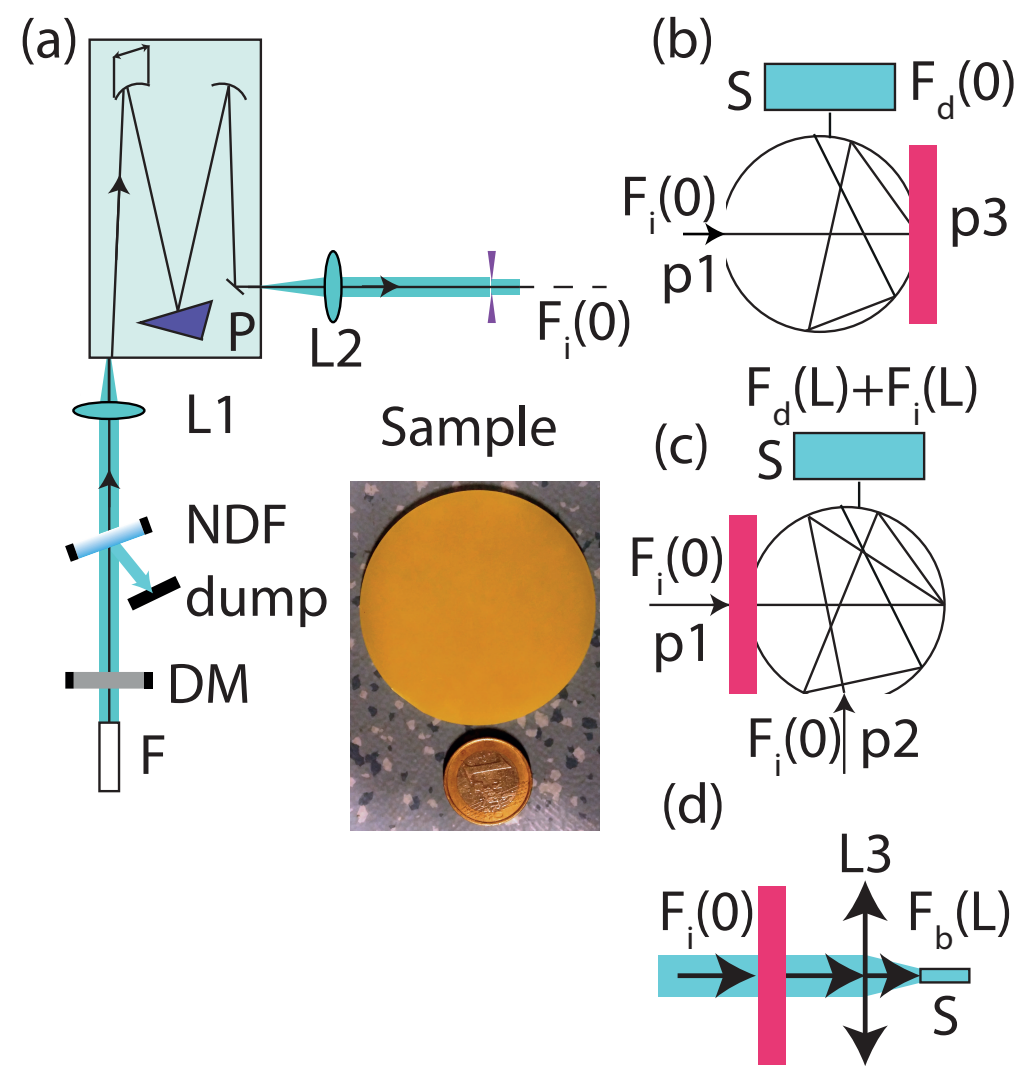

Figure 4.3: Experimental setup for measuring transport parameters. (a) The light source used in the experiment. (b) The configuration of the integrating sphere used for the scattered reflection measurements. (c) The configuration of the integrating sphere used for the total transmission measurements. (d) The configuration for ballistic Lamber-Beer measurements. F: Supercontinuum white light source, NDF: Neutral density filter, DM: Dichroic mirror, L1: Achromatic doublet (AC080-010-A-ML, $\mathrm{f}=10 \mathrm{~mm}$ ), L2: Achromatic doublet $(\mathrm{f}=50 \mathrm{~mm})$, L3: Achromatic doublet (AC254-050-A-ML, $\mathrm{f}=50 \mathrm{~mm})$, I: Integrating sphere, S: Spectrometer, P: Prism monochromator $\left(f_{\sharp}=4.6\right)$, p1: port1, p2: port 2, p3: port 3 .

The sample is attached to port 1 with the incident light flux $F_{\mathrm{i}}(\lambda, 0, \rho)$ illuminating the sample. The transmitted light is collected by the integrating sphere and spectrally resolved with the fiber spectrometer. In the transmission configuration, the reference flux $F_{\mathrm{i}}(\lambda, 0, \rho)$ is separately measured with the incident light entering sphere through port 2 .

The ballistic detector measures the transmitted ballistic light $T_{\mathrm{b}}$. The scheme of this detector is shown in Fig. 4.3(d). It consists of an achromatic lens ( $\mathrm{f}=50 \mathrm{~mm}$ ) that collects the transmitted light into the entrance of a fiber spectrometer (AvaSpec-USB2-ULS2048L). The distance between the detector and the sample is set to $50 \mathrm{~cm}$. If the detector is placed closer to the sample, the ballistic signal is compromised by a significant contribution from the diffuse scattered light. The contribution of the scattered light in the ballistic signal is 
estimated following Ref. 88 to be less than $1 \%$.

The measurements of the transmission $T$, the reflection $R$ and the ballistic transmission $T_{\mathrm{b}}$ are repeated 10 times at every wavelength, to obtain statistical information. Consequently, we estimated the error to be about $\Delta T / T=\Delta R / R=4$ percent point for transmission and reflection measurements, and about $\Delta T_{\mathrm{b}} / T_{\mathrm{b}}=1$ percent point for the ballistic transmission measurements. In Fig. 4.5 and Fig. 4.6 the error bars are within the symbol size. The estimated errors of the total transmission $T$ and the scattered reflection $R$ are propagating linearly when the transport parameters are calculated, and result in a 4 percent point error on the extracted transport parameters $\left(\sigma_{\mathrm{s}}, \sigma_{\mathrm{a}}, \mu\right)$.

\subsection{Simulations}

We perform Monte Carlo ray tracing simulations, in other words, Monte Carlo simulations of the radiative transfer equation as independent verification of our analytical model. The Monte Carlo ray tracing was carried out using a computational geometry similar to the schematic in Fig. 4.2 with normally incident monochromatic plane wave on a plane parallel slab with finite thickness $d$. The scattering parameters $\left(\ell_{\mathrm{s}}, \ell_{\mathrm{a}}, \mu\right)$ for the chosen wavelength are taken as the input parameters for the simulations. The light transport is simulated as follows 89,90 : A bunch of $N_{\mathrm{b}}=10^{4}$ random walkers (photons) is launched with initial angular coordinates $\left(\theta_{0}, \phi_{0}\right)=(0 \mathrm{rad}, 0 \mathrm{rad})$ representing the normally incident plane wave. The incident photon bunch undergoes a three-dimensional random walk inside the slab. The random walk consists of a set of connected rectilinear paths of lengths $\left\{d_{i}\right\}$, each of which is randomly picked from an exponential distribution $p\left(d_{i}\right)=\left(1 / \ell_{\mathrm{s}}\right) \exp \left(-d_{i} / \ell_{\mathrm{s}}\right)$. The scattering at the end of each path is simulated by choosing an angular coordinate $\left(\theta_{i}, \phi_{i}\right)$ following the Henyey-Greenstein phase function [89] to accommodate the scattering anisotropy $\mu$ of the scatterers. Absorption in the medium is incorporated through the exponential decrease of the photon number: the attenuation depends exponentially on the path length $d_{i}$ and the absorption mean free path $\ell_{\mathrm{a}}$. The random walk is terminated when the photon bunch is either completely absorbed, or it has arrived at an interface. The specular light reflection from the interface is described with Fresnel's law [19]. The amount of transmitted photons $N_{\text {tr }}$, reflected photons $N_{\text {ref }}$, or absorbed photon $N_{\text {abs }}$ weight is registered at the end of the walk. Simulated transmission is defined as $T^{\mathrm{s}} \equiv N_{\mathrm{tr}} / N_{\mathrm{b}}$, and simulated reflection as $R^{\mathrm{s}} \equiv N_{\text {ref }} / N_{\mathrm{b}}$. A total of about $N_{\mathrm{ph}}=2 \times 10^{4}$ photon bunches are launched to statistically compute the transmission and reflection for a given set of transport parameters $\left(\ell_{\mathrm{s}}, \ell_{\mathrm{a}}, \mu\right)$. The number of photons $N_{\mathrm{ph}}$ is chosen to ensure the convergence of 


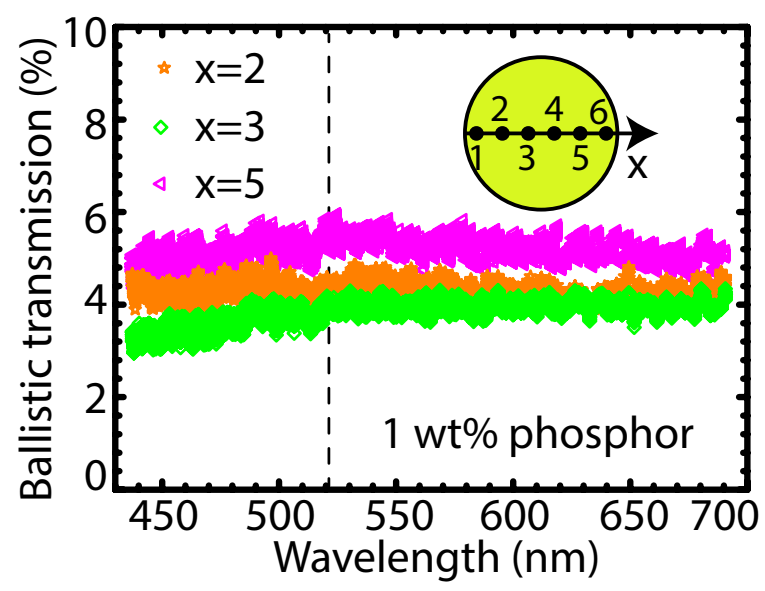

Figure 4.4: Ballistic transmission of the phosphor slab obtained in the experiment. of the silicone plate with $1 \mathrm{wt} \%$ of phosphor particles measured at different spatial positions. The beam position during the measurements is shown in the inset.

the algorithm within $1 \%$ of the transmission fluctuations between different algorithm runs. We scan the transport parameter range $\left(\mu \in[0,1], \sigma_{s} \in[1,3]\right.$ and $\left.\sigma_{a} \in[0,0.5]\right)$ for different phosphor concentrations and choose the transport parameters that yield the transmission and the reflection obtained in the experiment.

\subsection{Results}

\subsubsection{Ballistic transmission}

The samples with $1 \mathrm{wt} \%$ and $2 \mathrm{wt} \%$ phosphor concentration were used for the ballistic transmission measurements since they are optically sufficiently thin. The ballistic transmission for other plates is less than $0.1 \%$, which is below the signal to noise level of our detector at the available incident signal intensities.

We scan the incident wavelength $\lambda_{\mathrm{i}}$ from $420 \mathrm{~nm}$ to $700 \mathrm{~nm}$, and collect the ballistic light, with light incident at six different spatial positions on the sample as shown in the inset of Fig. 4.4. Representative data sets are shown in Fig. 4.4, data for positions 1,4, and 6 are in between the presented data sets. The ballistic transmission spectra for the slab with $1 \mathrm{wt} \%$ is shown at three selected spatial positions in Fig. 4.4. The ballistic transmission at wavelength $\lambda_{\mathrm{i}}$ differs at most by 2 percent points at different spatial locations $\Delta T_{\mathrm{b}} / T_{\mathrm{b}}<2 \%$. The difference between different data sets arises from the exponential sensitivity of the ballistic light to variations in the local phosphor particle concentration, see Eq. 4.7).

We extract the sum of the scattering and absorption cross sections $\sigma_{\mathrm{s}}+\sigma_{\mathrm{a}}$ from the measured ballistic transmission data using Eq. (4.7). The results are plotted in Fig. 4.5 


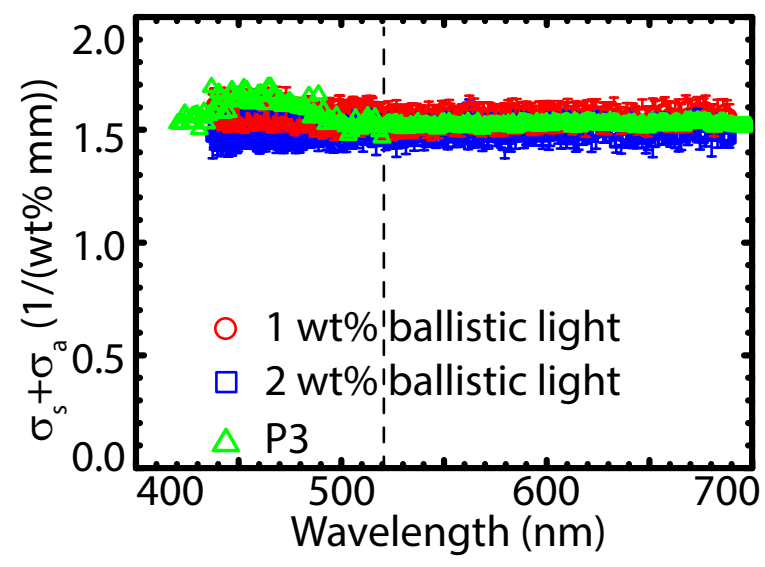

Figure 4.5: Measured scattering and absorption cross sections. The sum of the scattering cross section $\sigma_{\mathrm{s}}$ and absorption cross section $\sigma_{\mathrm{a}}$ measured as a function of wavelength for two different phosphor concentrations. Green triangles represent data extracted from the diffused light measurements.

for the samples with $1 \mathrm{wt} \%$ and $2 \mathrm{wt} \%$ concentrations of phosphor particles. We observe excellent agreement between samples with different concentrations of phosphor in the wavelength range of interest. In the non-absorbing range $(520 \mathrm{~nm}$ to $700 \mathrm{~nm})$, the extracted cross section is equal to the scattering cross section $\sigma_{\mathrm{s}}$ since the absorption cross section vanishes.

In the absorption range, the measured ballistic transmission is constant within the signal to noise ratio. From this behavior, we conclude that the absorption cross section $\sigma_{\mathrm{a}}$ is at least one order of magnitude smaller than the scattering cross section $\sigma_{\mathrm{s}}$. In the case of the multiple scattering even this small absorption has a crucial influence on the diffuse transmission, and reflection (see subsequent sections).

\subsubsection{Diffuse light transmission and reflection}

We measure the transmission and the reflection spectra for the slabs with different phosphor particle concentrations. The measured results are shown in Fig. 4.6(a) and 4.6(b). For visual clarity, we plot only a selection of the measured samples. The transmission and the reflection spectra reveal a deep trough with a minimum at $\lambda_{\mathrm{i}}=458 \mathrm{~nm}$. The trough matches the peak of the absorption band of YAG: $\mathrm{Ce}^{3+}[86$. The presence of the trough indicates that the phosphor absorbs a significant fraction of the light in this wavelength range. In the range $\lambda_{\ell}=490 \mathrm{~nm}<\lambda_{\mathrm{r}}<520 \mathrm{~nm}$ where absorption and emission of phosphor overlap (see Ch. 3] 86, the spectra show a steep rise in the transmission due to the reduced absorption. At wavelengths longer than $\lambda_{\mathrm{r}}=520 \mathrm{~nm}$, the transmission spectra are flat. Unlike the transmission spectra, the scattered reflection spectra depend only weakly on the phosphor 

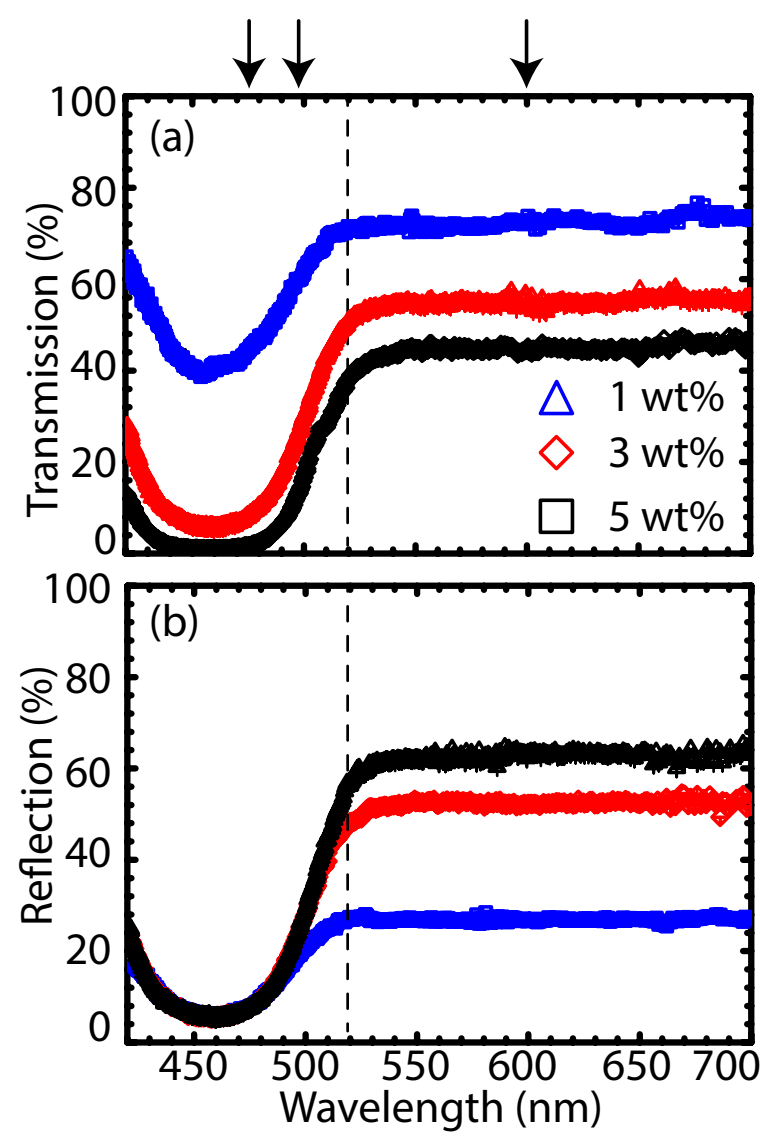

Figure 4.6: Transmission and scattered reflection spectra of silicone plates. (a) Broadband transmission $T$ as a function of concentration. (b) Broadband reflection as a function of concentration. Concentration of the phosphor particles is indicated on the figures. Arrows indicate the wavelengths for the Fig. 4.7

concentration $\rho$ in the absorption range up to $\lambda_{\ell}=490 \mathrm{~nm}$ Fig. 4.6(b).

The sum of the total transmission and the scattered reflection was found to be $104 \%$ for all the measurements in the non-absorbing range. This small systematic error could arise from an overestimation of the diffuse light signal. A possible source of the excess signal is the specular light (see Fig 4.3). Approximately $2 \%$ of the reference light is being specularly reflected (Fresnel reflection) from the sample and is lost in the setup. Part of the specularly reflected light can also illuminate the sample, however, resulting in an effectively higher flux that illuminates the sample.

We analyze experimental results by extracting the transport parameters using the P3 approximation model Eq. (4.4) and (4.5) to the total diffuse transmission and the total diffuse reflection data. Since the theoretical description requires energy conservation with the diffuse reflection and transmission adding to $100 \%$, we decided to fit $T$ and $R$ separately given the slight systematic error mentioned above. As an example, the fit at $\lambda_{\mathrm{i}}=599 \mathrm{~nm}$ 


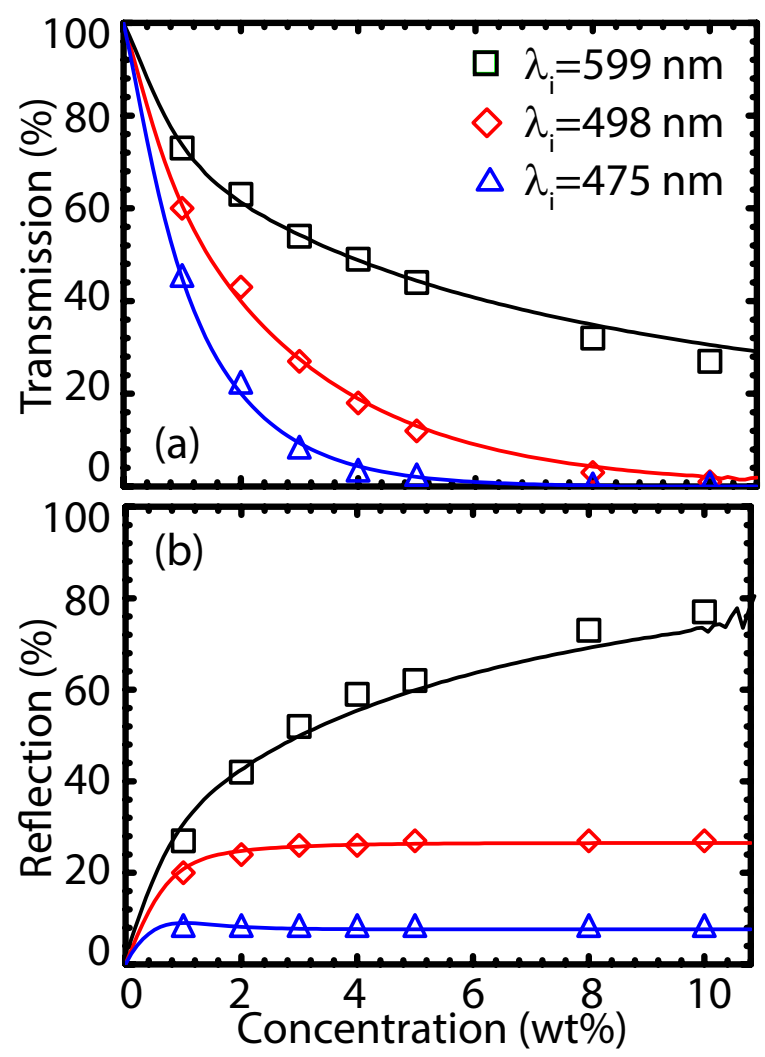

Figure 4.7: Transmission and reflection as a function of concentration for three chosen wavelengths. (a) Transmission as a function of concentration shown for three different wavelengths. (b) Reflection as a function of concentration shown for the same three different wavelengths. In both figures, symbols represent measured values. Solid lines are analytical results obtained using the P3 approximation to the radiative transfer equation.

is shown in Fig. 4.7. The difference between the anisotropy parameter $\mu(\lambda=599 \mathrm{~nm})$ extracted from $T$ and $R$ is about $4 \%$ percent, which is within the error of the experiment. Fig. 4.7 illustrates the model prediction at three wavelengths in the range of strong absorption $\left(\lambda_{\mathrm{i}}=475 \mathrm{~nm}\right)$, weak absorption $\left(\lambda_{\mathrm{i}}=498 \mathrm{~nm}\right)$, and no absorption $\left(\lambda_{\mathrm{i}}=599 \mathrm{~nm}\right)$. The transmission is a monotonically decaying function of the concentration $\rho$ over the studied wavelength range. The diffuse reflection is an increasing function of the concentration $\rho$ in the wavelength range with no absorption. In the absorption range, the increase of the reflection $R$ in the high concentration range is hampered by the absorption.

For each wavelength $\lambda_{\mathrm{i}}$ in the absorption range $\left(400<\lambda_{\mathrm{i}}<490 \mathrm{~nm}\right)$, we fit the analytic $\left(T^{\mathrm{m}}, R^{\mathrm{m}}\right)$ simultaneously to the measured $(\mathrm{T}, \mathrm{R})$ as a function of the concentration $\rho$, with the scattering cross section $\sigma_{\mathrm{s}}$, the absorption cross section $\sigma_{\mathrm{a}}$, and the anisotropy factor $\mu$ as the adjustable parameters. In the non-absorbing range, $490 \mathrm{~nm}<\lambda_{\mathrm{i}}<700 \mathrm{~nm}$ the scattering cross sections $\sigma_{\mathrm{s}}$ is taken from the ballistic light measurements. For the stability 
of the procedure, we take the absorption cross section to be small $\sigma_{\mathrm{a}}=10^{-7}$, where we verified that even a decrease of $\sigma_{\mathrm{a}}$ by several orders of magnitude does not affect the result. The only adjustable parameter in the non-absorbing range is the anisotropy factor $\mu$. If the total transmission has sharply decreased to $T<1 \%$, the analytical model reveals strong oscillations, as shown in Fig. 4.7(b). These oscillations are the result of finite machine precision in calculating the numerical values of the total transmission $T$, and the scattered reflection $R$ using Eq. 4.4 and 4.5 .

In Fig. 4.8 the extracted parameters $\left(\sigma_{\mathrm{s}}, \sigma_{\mathrm{a}}, \mu\right)$ are shown for the whole visible wavelength range. In the whole wavelength range the scattering cross section $\sigma_{\mathrm{s}}$, and the anisotropy factor $\mu$ remain constant with the incident varying wavelength $\lambda_{\mathrm{i}}$. In the absorption range, the maximum absorption cross section $\sigma_{\mathrm{a}}$ coincides with the absorption peak of the YAG: $\mathrm{Ce}^{3+}$. The absorption cross section tends to zero at the edges of the absorption spectral range $\left(\lambda_{\mathrm{i}}=420 \mathrm{~nm}\right.$ and $\left.520 \mathrm{~nm}\right)$ in agreement with [86. To compare the P3 approximation to the diffusion equation (P1 approximation), we determine the absorption cross section $\sigma_{\mathrm{a}}$ using both equations. The diffusion equation shows up to $30 \%$ error in the absorption cross section $\sigma_{\mathrm{a}}$, and hence cannot be used for strongly absorbing materials, see Fig. 4.8 (c).

To verify the derivation of the parameters $\left(\sigma_{\mathrm{s}}, \sigma_{\mathrm{a}}, \mu\right)$ by our analytical model, we employ Monte Carlo simulations of the radiative transfer equation. At every wavelength $\lambda$, we simulate $\left(T^{\mathrm{s}}, R^{\mathrm{s}}\right)$ as a function of the parameters $\left(\ell_{\mathrm{s}}, \ell_{\mathrm{a}}, \mu\right)$ to within $4 \%$ of the measured data $(T, R)$. For a particular concentration $\rho$ this comparison yields the parameters $\left(\ell_{\mathrm{s}}, \ell_{\mathrm{a}}\right.$, $\mu$ ) as a function of $\lambda$. We compute the parameter estimates for all measured concentrations $\rho$. We verify that $\left(\ell_{\mathrm{s}}, \ell_{\mathrm{a}}\right)$ are proportional to the density $\rho$, and that $\mu$ remains fixed for all densities $\rho$. The $\left(\sigma_{\mathrm{s}}, \sigma_{\mathrm{a}}, \mu\right)$ at a given wavelength $\lambda$ is the best fit parameter set for all concentrations. We convert $\left(\ell_{\mathrm{s}}, \ell_{\mathrm{a}}\right)$ to $\left(\sigma_{\mathrm{s}}, \sigma_{\mathrm{a}}\right)$ using Eq. 6.2 and Eq. 6.3.

The simulation results reveal good agreement with the analytical P3 results, as shown in Fig. 4.8, where the error bars in the Monte Carlo parameter estimate is a result of the regression analysis. Hence the transport parameters of white LED can be extracted equally well with the P3 approximation to the radiative transfer equation (RTE) as with the Monte Carlo simulations. We also compare the sum of the scattering cross section $\sigma_{\mathrm{s}}$ and the absorption cross section $\sigma_{\mathrm{a}}$ with the sum obtained from the ballistic light measurements in the absorption range also shown in Fig. 4.5. Both approaches yield a sum $\left(\sigma_{\mathrm{s}}+\sigma_{\mathrm{a}}\right)$ that is in excellent agreement. In the absorption range $(420<\lambda<520 \mathrm{~nm})$ the P3 approximation reveals a shallow peak that is not apparent in the ballistic data, most likely due to the limited signal to noise ratio in the latter. Thus, the transport parameters extracted with the P3 

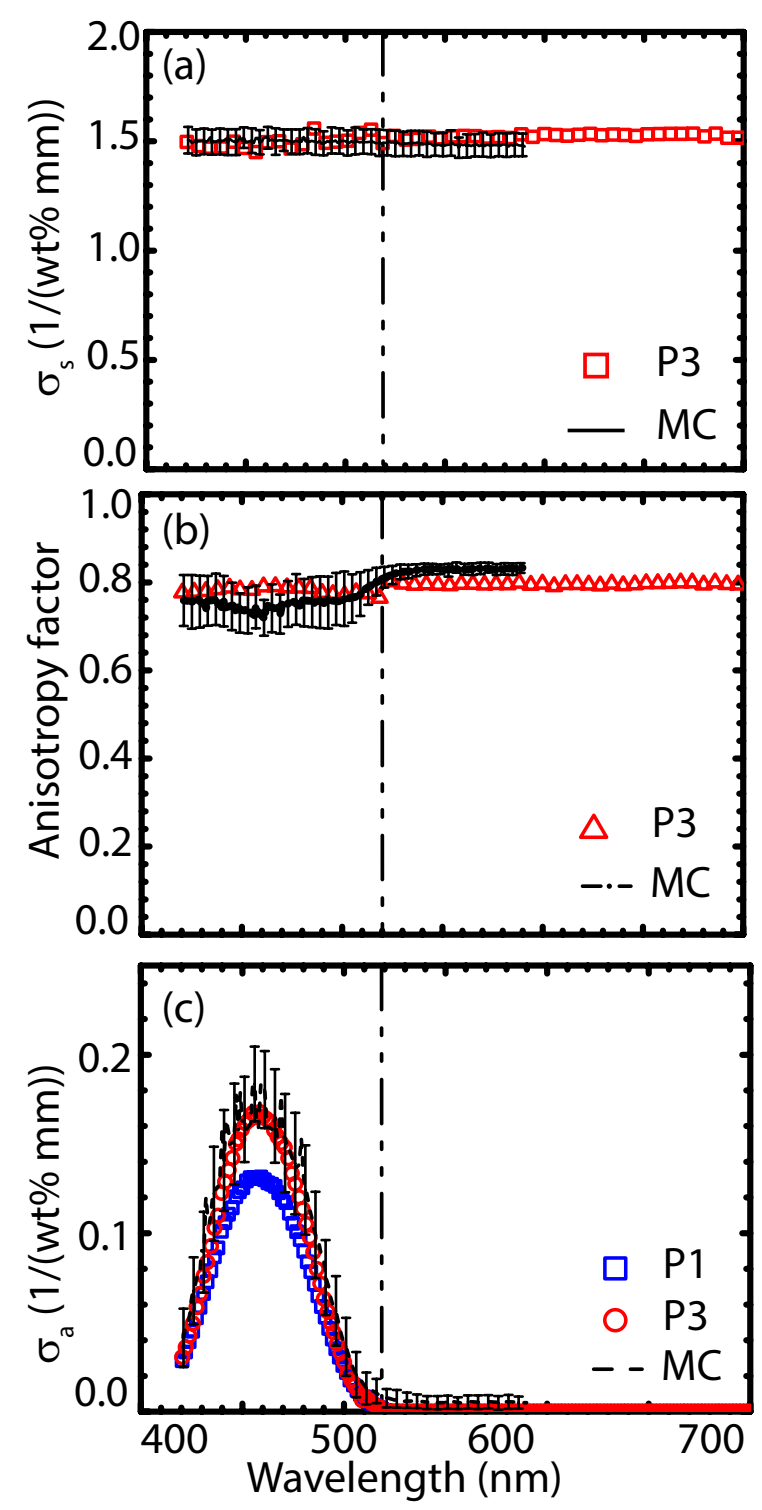

Figure 4.8: Transport parameters of YAG:Ce ${ }^{3+}$ phosphor particles extracted using analytical model and Monte Carlo simulations. Open symbols are the transport parameters values extracted using an analytical approach. The solid, dashed, dot-dashed lines are the Monte Carlo simulation results with respective error bars. (a) Scattering cross section $\sigma_{\mathrm{s}}$ as a function of wavelength. (b) Anisotropy factor $\mu$ as a function of wavelength.(c) Absorption cross section $\sigma_{\mathrm{a}}$ as a function of wavelength calculated using diffusion theory (P1), P3 approximation, and using Monte Carlo simulations.

approximation to the RTE are in good agreement with both the ballistic light determination and with the Monte Carlo estimations, which confirms the validity of our analytical model. 


\subsection{Discussion}

We verified that our analytical method is superior to numerical methods in speed and precision: It takes $\sim 1 \mathrm{~min}$ at each wavelength to obtain the transport parameters with error margins $\Delta \sigma_{\mathrm{s}} / \sigma_{\mathrm{s}} \leq 4 \%, \Delta \sigma_{\mathrm{a}} / \sigma_{\mathrm{a}} \leq 4 \%$ and $\Delta \mu / \mu \leq 4 \%$ with the analytical approach. The extraction speed of the analytical method can be further greatly optimized using dedicated solvers. The precision of the analytical method within the much shorter processing time is only limited by the precision of the measured input data. To achieve comparable precision it takes $\sim 17 \mathrm{~min}$ on conventional PC for a Monte Carlo approach with even larger error margins $\Delta \sigma_{\mathrm{s}} / \sigma_{\mathrm{s}} \leq 4 \%, \Delta \sigma_{\mathrm{a}} / \sigma_{\mathrm{a}} \leq 10 \%$ and $\Delta \mu / \mu \leq 7 \%$. The precision of the Monte Carlo simulations improves only relatively slowly as it scales as the square root of the number of rays used. Another advantage of the analytical approach over numerical methods is the predictive power over a broad range of transport parameters where $d / \ell_{\mathrm{a}}<7$.

Let us place our approach in the context of previous work on the analytical extraction of transport parameters. Leung et al. reported the transport properties of YAG:Ce ${ }^{3+}$ plates using a filtered broadband light source, where the linear dependence of $\ell_{\operatorname{tr}}$ was exploited to calculate $\ell_{\mathrm{a}}$ [54]. Firstly, the approximation used to analyze the total transmission was employed outside its range of validity. Secondly, their described method is only valid in the region of strong absorption or emission, and not in the overlap region. Hence the described approach could not be employed to extract transport properties over the whole visible spectral range.

Gaonkar et al. [91] used phenomenological theory (Kubelka-Munk theory) to decouple scattering and absorption properties of the diffusive samples. In the described approach the transport parameters are extracted using only an empirical relation between the KubelkaMunk and the radiative transfer equation. Moreover, they extracted only the transport mean free path $\ell_{\operatorname{tr}}$ and the absorption mean free path $\ell_{\mathrm{a}}$, which gives no explicit information about the anisotropy factor $\mu$.

In Ch. 3 (published earlier as Ref. [86]) we reported the transport properties of YAG:Ce ${ }^{3+}$ plates using a narrowband light source that works in the whole visible spectral range including the overlap range $\left(\lambda_{\ell}=490 \mathrm{~nm}<\lambda_{\mathrm{i}}<\lambda_{\mathrm{r}}=520 \mathrm{~nm}\right)$. We calculated $\ell_{\mathrm{a}}$ under the assumption that $\ell_{\operatorname{tr}}$ linearly depend on the wavelength in the absorption range, as was confirmed earlier on. Nevertheless, in the strongly absorbing region, the employed diffusion theory is not valid. Hence this method cannot adequately capture the parameter range of white LEDs (see Fig. 4.1). 


\subsection{Summary and outlook}

We have investigated the transport of light through slabs that scatter and strongly absorb, with a focus on white LEDs. I extracted the transport parameters of white LED phosphor plates within the visible wavelength range. We measured total transmission, scattered reflection, and ballistic transmission over the whole visible wavelength range. I analyzed the diffuse light measurements using the P3 approximation to the radiative transfer equation to extract the scattering cross-section $\sigma_{\mathrm{s}}$, the absorption cross-section $\sigma_{\mathrm{a}}$ and the anisotropy factor $\mu$, in scattering media with strong absorption, up to $d / \ell_{\mathrm{a}}=4$. The Lambert-Beer law was used to analyze the ballistic light measurements, and extract $\sigma_{\mathrm{s}}+\sigma_{\mathrm{a}}$ in the whole visible spectral range. I verified the data obtained with the diffuse light measurements to the data measured with the ballistic light, and Monte Carlo simulations. Our study confirms the validity of the P3 approximation for light propagation in a medium suitable for white LEDs (see Fig. 4.1).

The procedure of finding transport parameters is a significant hindering step for the conventional light propagation modeling, such as ray tracing [15 18,76]. Our method avoids the main bottleneck of these conventional numerical methods, providing at least 17-fold speed-up in time with the same error (see Fig. 4.8). The described approach is a suitable analytical tool for the industry since it provides a fast yet accurate determination of key transport parameters, and more importantly, it introduces predictive power into the design process of white LEDs. Both the know-how of and the control over optics that strongly scatters light has dramatically advanced $23,28,92]$. The state-of-the-art is much less developed regarding optical systems that also strongly absorb light (or even re-emit light of a different color), even though essential application fields occur in this regime. Examples are biomedical optics [32 37], or powder technology [38 40]. In this chapter, we provide a tool that also can be used in the fields listed above. 


\section{Chapter 5}

\section{Predicting the color of white LEDs}

\subsection{Introduction}

In spite of the fast-growing market penetration of white LEDs devices, there are only a trial and error approaches for their design. Here we make a paradigm shift in white LED design, by introducing a radically new design principle for white LED. Our design principle predicts the color point [55] as a function of the design parameters and shows excellent agreement with our experimental observations. The proposed method allows exploring a large parameter space as it pertains to any phosphor and any polymer used in white LED production. Our model provides for the fast and efficient design, resulting in a reduction of both design and production costs. Widespread adoption of our design approach will lead to a dramatically increased proliferation of efficient white light sources, even in remote locations with little technological infrastructure.

The main characteristic of a white light source is the color point that is described using color coordinates in the color space shown in Fig. 5.1 93, 94]. The rim of the color space indicates pure monochromatic colors, and space is filled with all mixtures of monochromatic colors. By choosing the proper mix of monochromatic colors, one designs the color point of any white light source. Optical designers in an industry currently use extensive numerical simulations 95,96 to extract the color point from the design parameters of the white light source. Conversely, to target a specific color point, optical designers use a trial and error Monte Carlo ray tracing approach. To systematically design the color point of a white LED one needs to connect the design parameters of a white LED to the color point.

To adjust the color point one can control several design parameters such as the phosphor layer thickness $d$, the phosphor concentration $\rho$, the phosphor type, the type of blue LED, the additional scattering elements. In this chapter we demonstrate an analytical method to set the design parameters of a white LED starting from a targeted color point or conversely, 


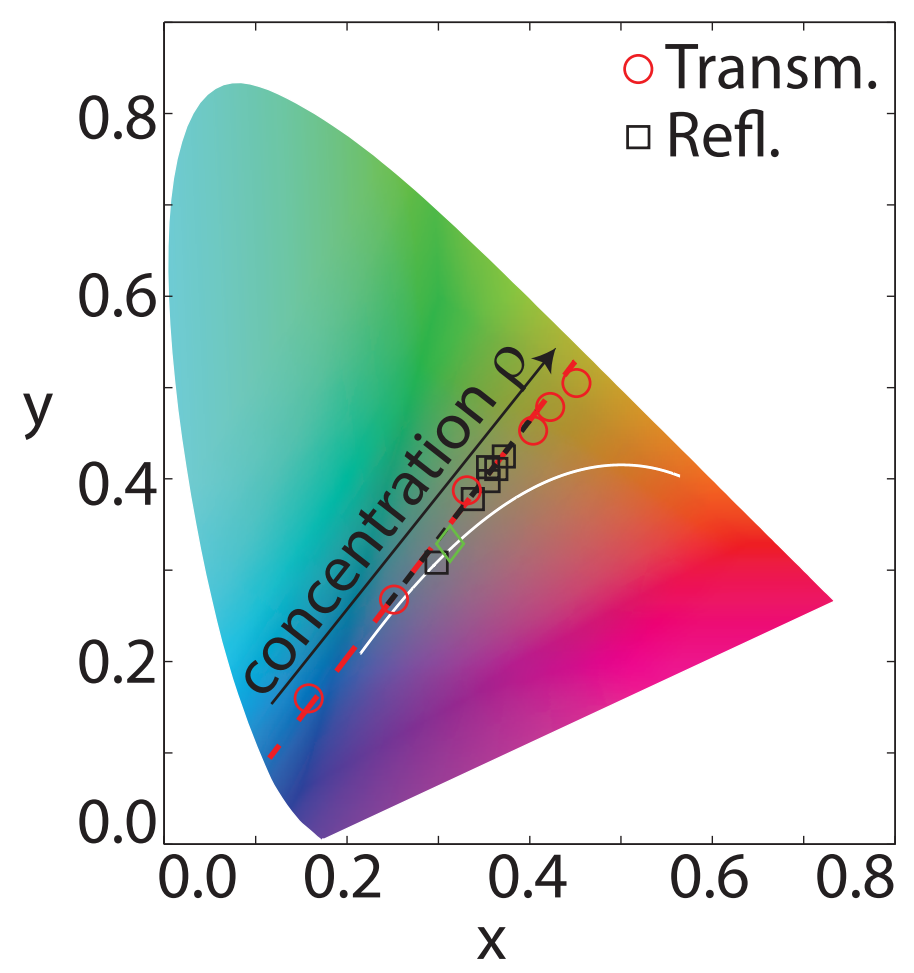

Figure 5.1: Color point of a white LED. The colored area indicates x-y color space 93]. Red and black dashed lines represent predicted color point dependence (curves in Fig. 5.4) on the phosphor concentration $\rho$ (1 wt $\%$ to $8 \mathrm{wt} \%)$ for transmitted and reflected light, respectively. Circles and squares are our experimental data points. The green diamond indicates the most widely used standardized white light spectrum - D65 spectrum. The white curve represents the Planckian locus of blackbody temperature.

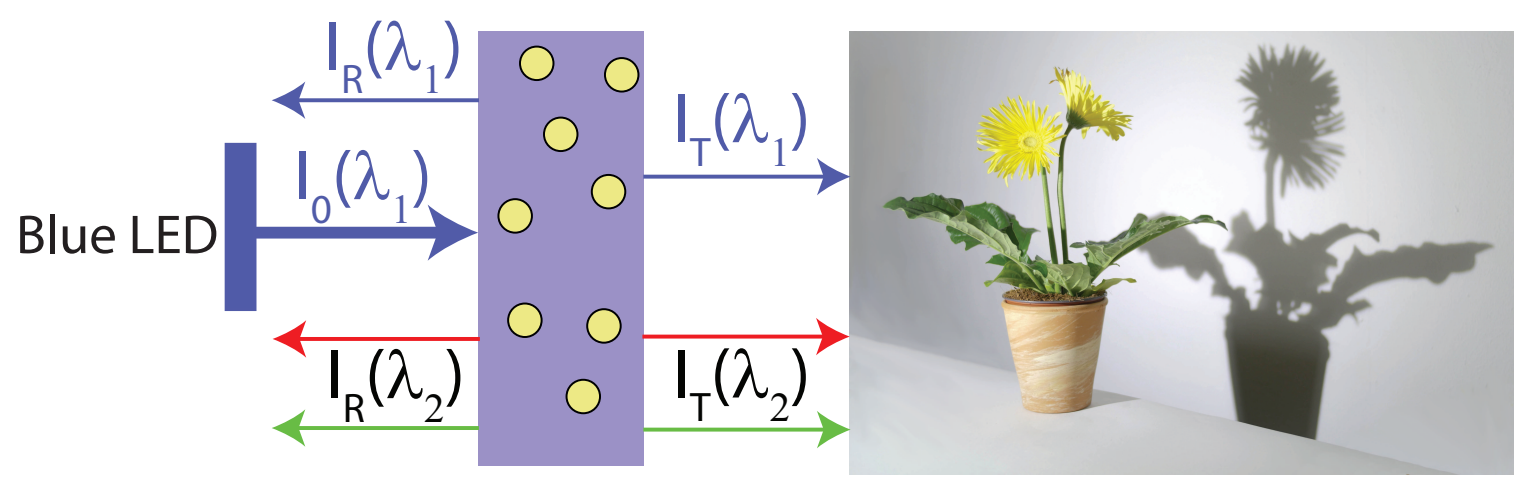

Figure 5.2: Model for light propagation in a white LED slab. Incident blue light with flux $F_{0}\left(\lambda_{1}\right)$ is shone by the blue LED on the phosphor slab with thickness d. The phosphor slab contains phosphor microparticles that are represented by yellow circles. $F_{\mathrm{T}}\left(\lambda_{1}\right)$ is the total transmitted flux, $F_{\mathrm{R}}\left(\lambda_{1}\right)$ is the scattered reflected flux, $F_{\mathrm{T}}\left(\lambda_{2}\right)$ is the transmitted reemitted flux, and $F_{\mathrm{R}}\left(\lambda_{2}\right)$ is the reflected re-emitted flux. The mixture of transmitted red, green and blue light illuminates the object. 


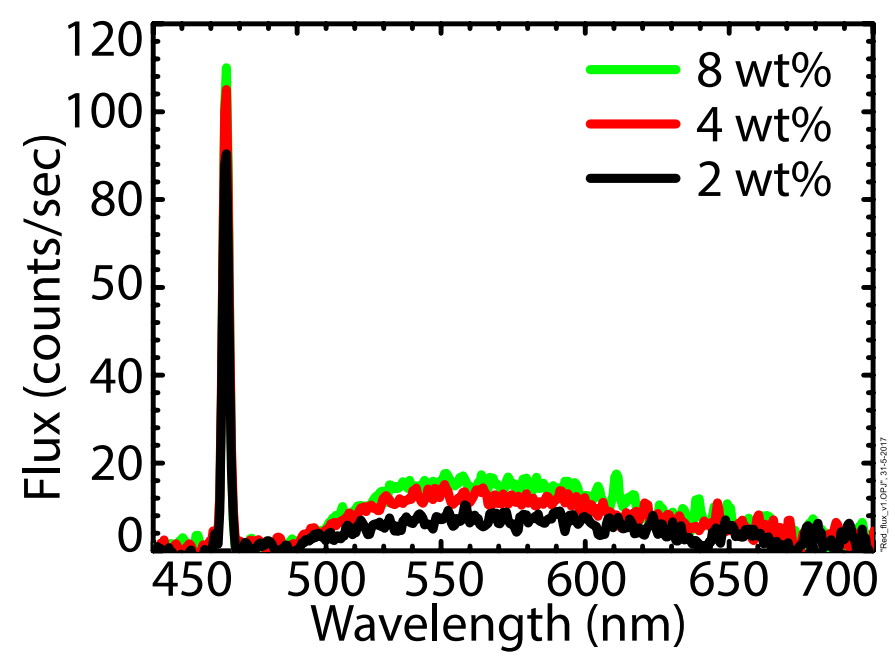

Figure 5.3: Representative measured signal of a model white LED. The sharp peak at $\lambda_{1}=475 \mathrm{~nm}$ is the scattered incident light in reflection $F_{\mathrm{R}}\left(\lambda_{1}\right)$, and the broad peak between $490 \mathrm{~nm}$ and $700 \mathrm{~nm}$ is light re-emitted by the phosphor $F_{\mathrm{R}}\left(\lambda_{2}\right)$. Our model is not limited to the choice of the blue LED wavelength.

to predict the color point of a white LED starting from the chosen parameters. Based on the developed model we derived the expressions for the total flux emitted in the forward direction by model white LED with reflecting mirror

\subsection{Methods}

Our analytical model describes all light fluxes emanating from a white LED (see Fig. 5.2. We study light propagation through a model white LED that consist of a polymer diffuser plate containing YAG: $\mathrm{Ce}^{3+}$ phosphor microparticles. The refractive index of the diffuser plates is $n=1.4$, and the plate thickness $d=1.98 \pm 0.02 \mathrm{~mm}$. The absorption and the emission spectra of the phosphor are discussed in Ch. 3. The phosphor particle concentration $\rho$ ranges from $1 \mathrm{wt} \%$ to $8 \mathrm{wt} \%$. A tunable blue light source with flux $F_{0}\left(\lambda_{1}\right)$ illuminates the samples at a wavelength $\lambda_{1}$ see Ch. 3. The incident light is scattered, absorbed, and re-emitted resulting in the transmitted blue flux $F_{\mathrm{T}}\left(\lambda_{1}\right)$, the reflected flux $F_{\mathrm{R}}\left(\lambda_{1}\right)$, the reemitted flux in transmission $F_{\mathrm{T}}\left(\lambda_{2}\right)$, and the re-emitted flux in reflection $F_{\mathrm{R}}\left(\lambda_{2}\right)$, where $\lambda_{2}$ is the wavelength of the re-emitted light. The transmitted and reflected fluxes are separately collected with an integrating sphere and detected with a fiber spectrometer.

The typical signal of our model white LED is shown in Fig. 5.3. The sharp peak at $\lambda_{1}=475 \mathrm{~nm}$ originates from the blue source and contributes to the light fluxes $F_{\mathrm{T}}\left(\lambda_{1}\right)$ and $F_{\mathrm{R}}\left(\lambda_{1}\right)$. The broad peak between $490 \mathrm{~nm}$ and $700 \mathrm{~nm}$ consists of the light fluxes $F_{\mathrm{T}}\left(\lambda_{2}\right)$ and $F_{\mathrm{R}}\left(\lambda_{2}\right)$. From the experiment we derive the following quantities that will be analytically 
modeled, namely the total transmission $T\left(\lambda_{1}\right)=F_{\mathrm{T}}\left(\lambda_{1}\right) / F_{0}\left(\lambda_{1}\right)$, the re-emitted transmission $T_{\mathrm{r}}\left(\lambda_{1}, \lambda_{2}\right)=F_{\mathrm{T}}\left(\lambda_{2}\right) / F_{0}\left(\lambda_{1}\right)$, the scattered reflection $R\left(\lambda_{1}\right)=F_{\mathrm{R}}\left(\lambda_{1}\right) / F_{0}\left(\lambda_{1}\right)$, and the re-emitted reflection $R_{\mathrm{r}}\left(\lambda_{1}, \lambda_{2}\right)=F_{\mathrm{R}}\left(\lambda_{2}\right) / F_{0}\left(\lambda_{1}\right)$. The transmissions $T\left(\lambda_{1}\right), T_{\mathrm{r}}\left(\lambda_{1}, \lambda_{2}\right)$ and the reflections $R\left(\lambda_{1}\right), R_{\mathrm{r}}\left(\lambda_{1}, \lambda_{2}\right)$ are obtained as a ratio of the areas under the scattered and re-emitted signal, as shown in Fig. 5.3 .

\subsection{Results and Discussion}

The experimental results for the scattered blue light are shown in Fig. 5.4 (insets). The transmission $T\left(\lambda_{1}\right)$ of the scattered light reveals a sharp decrease with the phosphor concentration $\rho$ as a result of scattering and strong absorption of the incident light. The reflection $R\left(\lambda_{1}\right)$ hardly varies with the phosphor concentration due to absorption; if the phosphor did not absorb light, we would have observed growth of the reflection with the increasing phosphor concentration, but the absorption counters this increase.

The measured transmission $T_{\mathrm{r}}\left(\lambda_{1}, \lambda_{2}\right)$ and reflection $R_{\mathrm{r}}\left(\lambda_{1}, \lambda_{2}\right)$ of the re-emitted light are shown in Fig. 5.4 (a)-(b). At low concentration, the re-emitted light flux $T_{\mathrm{r}}\left(\lambda_{1}, \lambda_{2}\right)$ increases with the phosphor concentration, because more phosphor absorbs more light. Saturation occurs at $\rho=3.3 \mathrm{wt} \%$ because a further increase of the concentration increases the probability of a photon being absorbed very close to the entrance surface of the scattering material. When most photons are absorbed near the entrance surface, then most re-emitted photons are back reflected resulting in a decrease of the transmitted re-emitted flux, and an increase of the reflected re-emitted flux.

The transmission $T\left(\lambda_{1}\right)$ and reflection $R\left(\lambda_{1}\right)$ of the scattered light are analytically calculated using the P3 approximation [33, 35, 36] to the radiative transfer equation (see Ch. 2] [42]. Input parameters of the solution are the slab thickness $d$, the particle concentration $\rho$, the scattering cross section $\sigma_{\mathrm{s}}\left(\lambda_{1}\right)$, the absorption cross section $\sigma_{\mathrm{a}}\left(\lambda_{1}\right)$ and the anisotropy factor $\mu\left(\lambda_{1}\right)$ of the phosphor particles. The optical designer chooses the first two parameters, and the last three parameters can be obtained for the whole visible spectral range with an experimental method described elsewhere [91, 97] or from prior knowledge on the phosphors 41. To describe the re-emitted light we calculate the absorbed average light flux distribution $U(z)$ inside the slab [43] (see Fig. 5.4 (c)). The average flux $U(z)$ is used as a source function for the re-emitted light that is also modeled by the P3 approximation w8 weighted by the quantum yield $q\left(\lambda_{1}, \lambda_{2}\right)$. The quantum yield is the ratio of the 

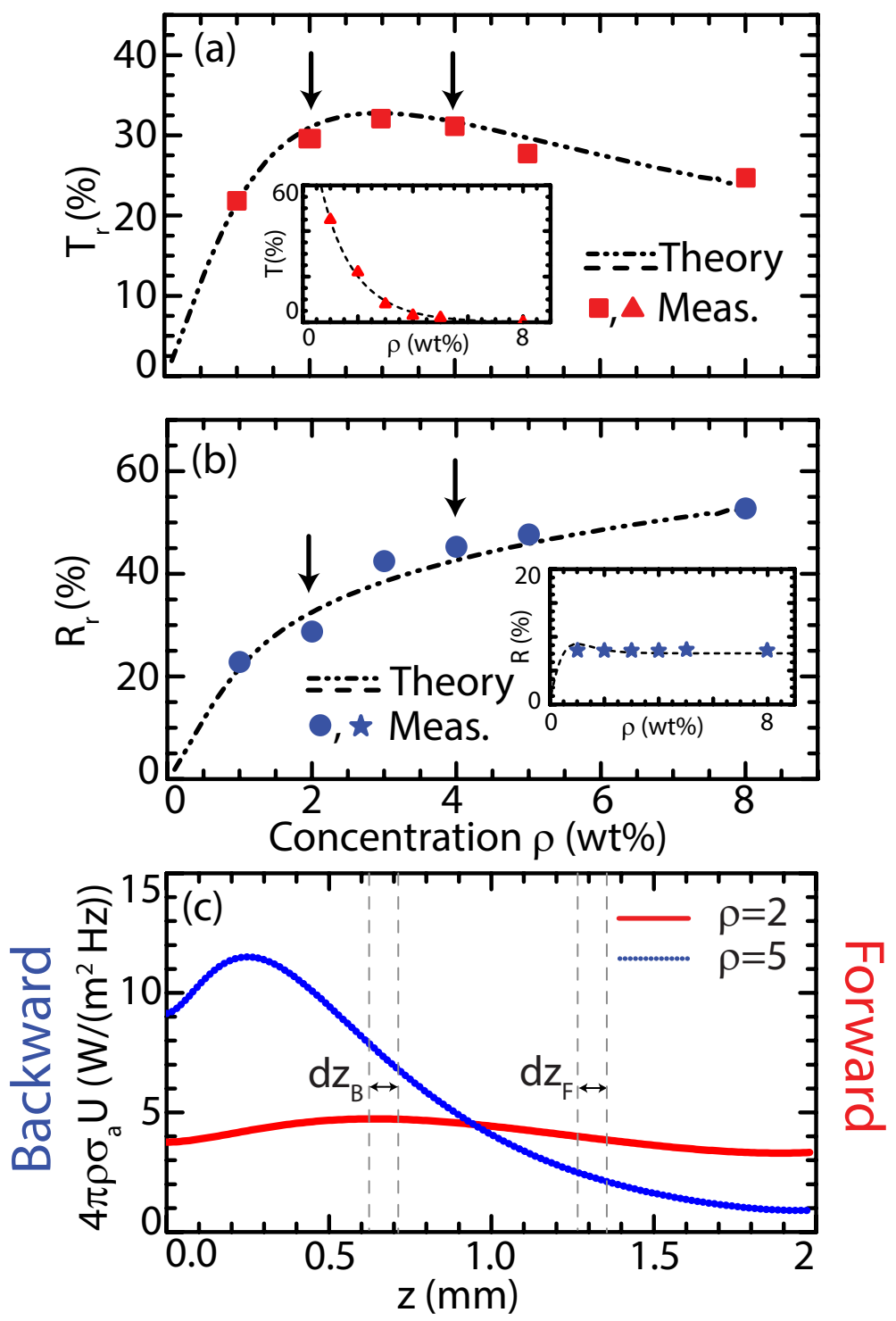

Figure 5.4: Transmission and reflection as a function of phosphor particle concentration. (a) Squares represent the measured transmission of the re-emitted light, dashed line represent predicted transmission of the re-emitted light. (b) Circles represent the measured reflection of the re-emitted light, dashed-dot-dot line represent predicted reflection of the re-emitted light. The error bars of the experiment are within the symbol size, and here $T_{\mathrm{r}}=\int_{490 \mathrm{~nm}}^{700 \mathrm{~nm}} F_{\mathrm{T}}(\lambda) d \lambda / F_{0}\left(\lambda_{1}\right), R_{\mathrm{r}}=\int_{490 \mathrm{~nm}}^{700 \mathrm{~nm}} F_{\mathrm{R}}(\lambda) d \lambda / F_{0}\left(\lambda_{1}\right)$. (c) Lines represent the average flux $U(z)$ as a function of depth inside the sample with thickness $2 \mathrm{~mm}$ for two different phosphor particle concentrations $\rho$ indicated with the black arrows in panels (a)-(b). Two representative ranges highlighted with gray dashed lines are shown at $d / 3$ and $2 d / 3$ depth in the sample. Colored labels "Backward" and "Forward" indicate the backward and forward light propagation directions. The insets in panels (a)-(b) show data and theory for the scattered light, compare Fig. 4.7.

total number of re-emitted photons to the total number of absorbed photons 99,100

$$
q\left(\lambda_{1}, \lambda_{2}\right) \equiv\left(T_{\mathrm{r}}\left(\lambda_{1}, \lambda_{2}\right)+R_{\mathrm{r}}\left(\lambda_{1}, \lambda_{2}\right)\right) /\left(1-\left(T\left(\lambda_{1}\right)+R\left(\lambda_{1}\right)\right)\right)
$$


We extracted quantum yield $q\left(\lambda_{1}, \lambda_{2}\right)$ using data shown in Fig. 5.4 (a-b). The broadband measurement of the quantum yield $q\left(\lambda_{1}, \lambda_{2}\right)$ is shown in (Fig. 5.5. We see that quantum yield increases when we shift to the higher wavelength. The quantum yield saturates at a wavelength $\lambda=444 \mathrm{~nm}$, and oscilates around the value of $\sim 0.85$, comparable to the previously reported values 101, 102 .

For the re-emitted light the P3 approximation to the radiative transfer equation is solved to extract the transmission $T_{\mathrm{r}}\left(\lambda_{1}, \lambda_{2}\right)$ and reflection $R_{\mathrm{r}}\left(\lambda_{1}, \lambda_{2}\right)$. Input parameters include the same parameters for the scattered light mentioned above, in addition the scattering cross section $\sigma_{\mathrm{s}}\left(\lambda_{2}\right)$, the absorption cross section $\sigma_{\mathrm{a}}\left(\lambda_{2}\right)$, the anisotropy factor $\mu\left(\lambda_{2}\right)$, and the quantum yield $q\left(\lambda_{1}, \lambda_{2}\right)$ of the phosphor particles.

The results of analytical solutions of the P3 approximation for the transmissions $T\left(\lambda_{1}\right)$ and $T_{\mathrm{r}}\left(\lambda_{1}, \lambda_{2}\right)$ and the reflections $R\left(\lambda_{1}\right)$ and $R_{\mathrm{r}}\left(\lambda_{1}, \lambda_{2}\right)$ as function of concentration $\rho$ are shown in Fig. 5.4 (a)-(b). We find an excellent agreement between the experiment and our analytic model. To explain the behaviour of the $T_{\mathrm{r}}\left(\lambda_{1}, \lambda_{2}\right)$ and $R_{\mathrm{r}}\left(\lambda_{1}, \lambda_{2}\right)$ as a function of phosphor concentration we plot the absorbed power $4 \pi \rho \sigma_{\mathrm{a}} U(z)$ as a function of depth inside the scattering medium in Fig. 5.4(c), where the $z$ coordinate corresponds to the notations in Fig. 5.2. When the concentration of the phosphor particles is low, the absorbed power is distributed almost uniformly across the sample. Re-emitted light generated as a result of absorption in a representative layer $d z_{\mathrm{B}}$ contributes mostly to the re-emitted reflection $R_{\mathrm{r}}\left(\lambda_{1}, \lambda_{2}\right)$ because the light that is re-emitted in the backward direction in this layer will experience less scattering compared to the forward re-emitted light. The reemitted light in a second representative layer $d z_{\mathrm{F}}$ will mostly contribute to $T_{\mathrm{r}}\left(\lambda_{1}, \lambda_{2}\right)$ for the same reason. When the phosphor concentration is high, the flux is mostly absorbed near the entrance surface of the slab. Hence, layer $d z_{\mathrm{B}}$ will contribute a higher flux in the backward direction compared to the low concentration case. The layer $d z_{\mathrm{F}}$ will contribute less flux in transmission $T_{\mathrm{r}}\left(\lambda_{1}, \lambda_{2}\right)$ compared to the low concentration case. This interplay results in the peak in transmission $T_{\mathrm{r}}\left(\lambda_{1}, \lambda_{2}\right)$ at $\rho=3.3 \mathrm{wt} \%$ and a steady increase of reflection $R_{\mathrm{r}}\left(\lambda_{1}, \lambda_{2}\right)$ with concentration $\rho$.

The color points for the measured data and our model are plotted in the color space in Fig. 5.1. The theoretical curves indicate all possible color points that can be achieved with the given YAG: $\mathrm{Ce}^{3+}$ phosphor when the concentration $\rho$ is changed. The observed dependence of the color point on concentration is remarkably linear given the observation above Fig. 5.4 that the scattered and re-emitted light fluxes depend non-linearly on the concentration. The color point linearly shifts to the yellow part of the color space with increasing concentration for both the transmitted and reflected light in Fig. 5.1. The reason 


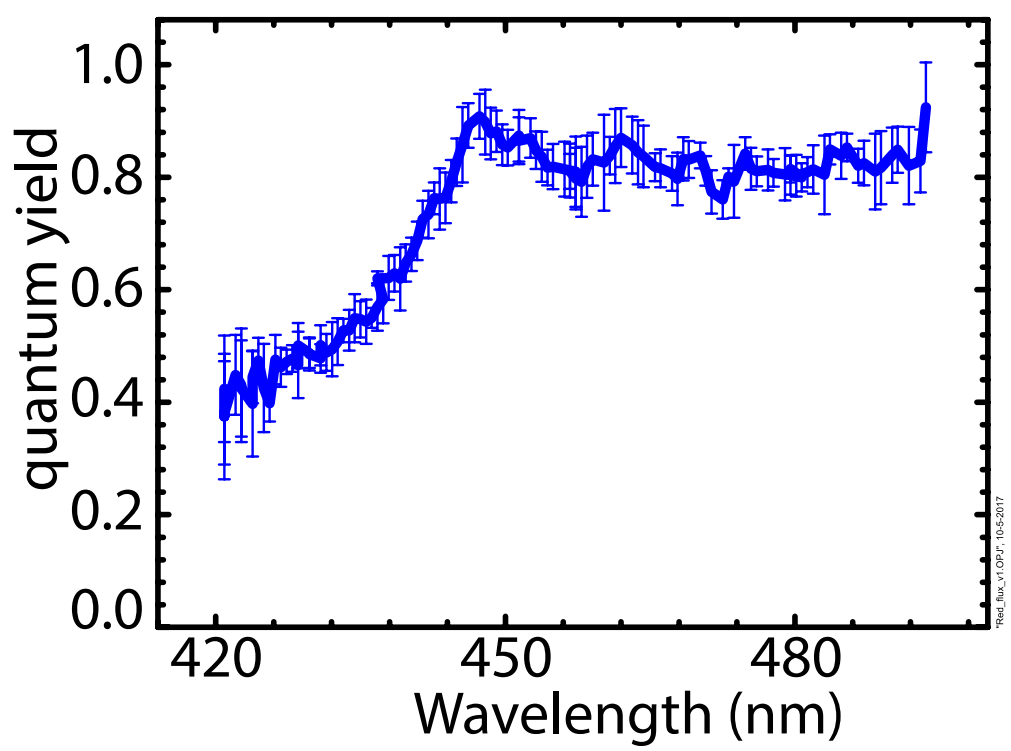

Figure 5.5: Quantum yield of YAG:Ce ${ }^{3+}$. Broadband quantum yield as a function of wavelength extracted from our measurements.

is that a higher concentration enhances the absorption of blue light increasing the produced re-emitted light.

\subsection{White LED with backreflector}

As a next step, we present a more complex model of a white LED that is relevant for several practical devices. In the commercially available white LED semiconductor and polymer are mounted in the cup covered with a thin layer of silver. Silver works as a mirror that reflects backscattered light back into the system and enhances the efficiency of the light source.

To account for this silver layer in our model, we place the mirror with reflectivity $R_{\mathrm{m}}$ on the left side of the polymer slab (see Fig. 5.6(b)). This mirror reflects the backscattered light at all wavelength. To calculate the resulting light flux emitted by the white LED we derive equations for the light flux using the iterative procedure. In the first stage, we illuminate the polymer slab with the excitation light flux $F_{0}$. The light is being scattered, absorbed and re-emitted by the phosphor in all directions. In Fig. 5.6.(b) dashed box of stage 1 scattering encloses all resulting fluxes produced by the incident flux $F_{0}$. The excitation light flux emitted in the backward direction is equal to $F_{0} R$, where $R$ is the reflectance of the polymer slab. The re-emitted light flux emitted in the backward direction is equal to $F_{0} R_{\mathrm{r}}$, where $R_{\mathrm{r}}$ is the reflectance of the polymer slab for the re-emitted light. The fluxes exciting on the right side of the sample are $F_{0} T$, and $F_{0} T_{\mathrm{r}}$ for excitation and re-emission respectively, where $T$ is the transmission of the slab and $T_{\mathrm{r}}$ is the transmission of the re-emitted flux. 

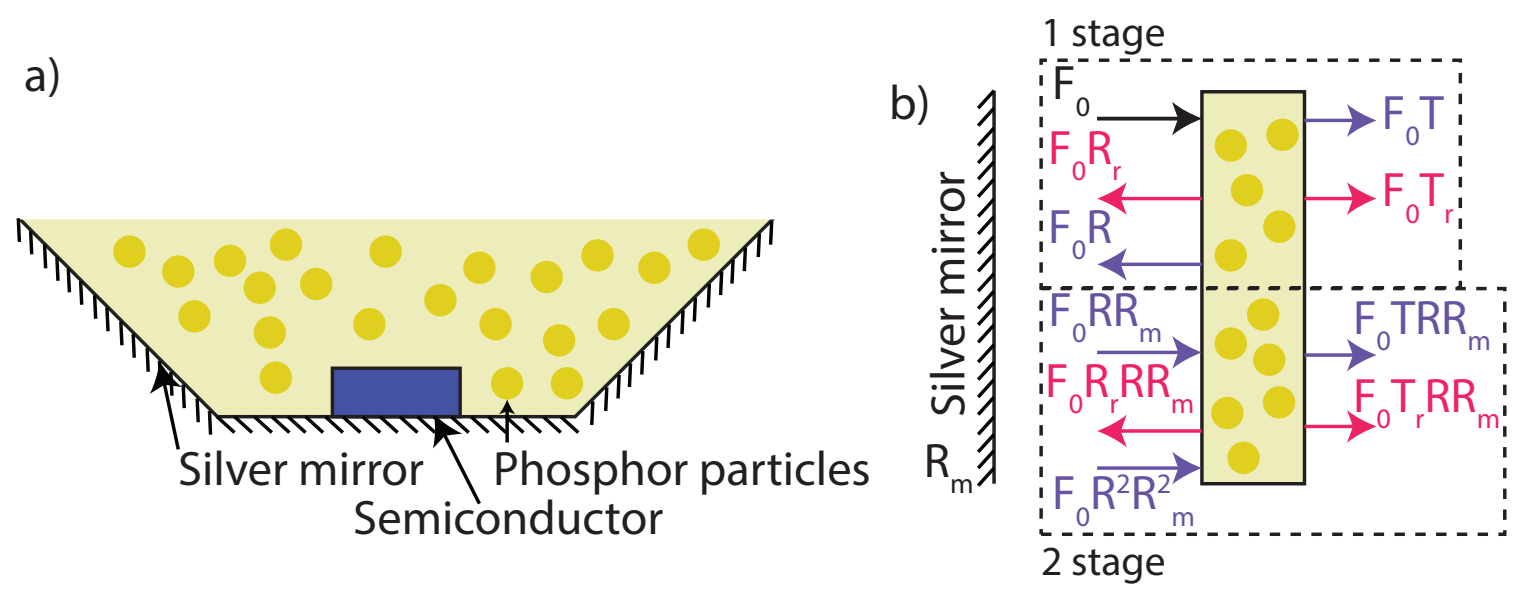

Figure 5.6: White LED with back-reflector. (a) Scheme of the white LED with backreflector. Blue rectangle is a semiconductor. Yellow circles are the phosphor inclusions. On the bottom and the sides of the LED cup silver mirror is located. (b) Silver mirror reflects the excitation light and the re-emission light that was backscattered by polymer plate with phosphor particles. $R$ is the reflectivity of the excitation light, $R_{\mathrm{r}}$ is the reflectivity of the re-emitted light. Red arrows represent the re-emitted flux, and blue arrows represent the excitation flux. Dashed boxes enclose stage 1 and 2 of the scattering and re-emitting processes.

The part of the backscattered light flux $F_{0} R R_{\mathrm{m}}$ is being reflected from the mirror and again illuminates the slab. This is the second stage of the scattering that is indicated in Fig. 5.6(b). The transmitted fluxes are $F_{0} T R R_{\mathrm{m}}$ and $F_{0} T_{\mathrm{r}} R R_{\mathrm{m}}$ for excitation and reemission, respectively. The backscattered fluxes are $F_{0} R^{2} R_{\mathrm{m}}^{2}$ and $F_{0} R_{\mathrm{r}} R R_{\mathrm{m}}$ for excitation and re-emission, respectively. This process of reflection from the mirror, scattering and re-emission continue to infinity. For the excitation light, the total transmitted light flux is calculated as

$$
F_{\text {tot }}=F_{0} T+F_{0} T R R_{\mathrm{m}}+F_{0} T R^{2} R_{\mathrm{m}}^{2}+\cdots=F_{0} T \sum_{n=0}^{\infty} R^{n} R_{\mathrm{m}}^{n}=F_{0} T \frac{1}{1-R R_{\mathrm{m}}},
$$

where we used the equation of the geometric sum. In the limit of the perfect mirror $\left(R_{\mathrm{m}}=\right.$ $1)$, and non-absorbing particles $\left(\sigma_{\mathrm{a}}=0\right)$ Eq. 5.2 becomes

$$
F_{\text {tot }}=F_{0} \frac{T}{1-R}=F_{0}
$$

where we used the fact that in the non-absorbing medium $T=1-R$. All the light eventually gets through the sample.

The transmitted re-emitted light is calculated in the same way as transmitted excitation 
light

$$
F_{\mathrm{T}}^{\mathrm{re}-\mathrm{em}}=F_{0} T_{\mathrm{r}}+F_{0} T_{\mathrm{r}} R R_{\mathrm{m}}+F_{0} T_{\mathrm{r}} R^{2} R_{\mathrm{m}}^{2}+\cdots=F_{0} T_{\mathrm{r}} \sum_{n=0}^{\infty} R^{n} R_{\mathrm{m}}^{n}=F_{0} T_{\mathrm{r}} \frac{1}{1-R R_{\mathrm{m}}},
$$

we replaced $T$ with $T_{\mathrm{r}}$ in this equation. This is not all re-emitted flux that is emitted on the right side of the sample. The backreflected re-emitted flux $F_{\mathrm{b}}$ is being reflected from the mirror and partially transmitted without absorption on the right side of the sample. To calculate the total flux emitted on the right side of the sample we need to account for this contribution. In order to calculate this contribution we first calculate the total backreflected re-emitted flux $F_{\mathrm{b}}$. The total backreflected re-emitted light flux is calculated in the same way as Eq. 5.4 by replacing $T_{\mathrm{r}}$ with $R_{\mathrm{r}}$

$$
F_{\mathrm{b}}=F_{0} R_{\mathrm{r}}+F_{0} R_{\mathrm{r}} R R_{\mathrm{m}}+F_{0} R_{\mathrm{r}} R^{2} R_{\mathrm{m}}^{2}+\cdots=F_{0} R_{\mathrm{r}} \sum_{n=0}^{\infty} R^{n} R_{\mathrm{m}}^{n}=F_{0} R_{\mathrm{r}} \frac{1}{1-R R_{\mathrm{m}}},
$$

where $R_{\mathrm{r}}$ defines the amount of the re-emitted light generated inside the slab and emitted in the backward direction. The generated re-emitted light flux $F_{\mathrm{b}}$ is being reflected by the mirror $\left(F_{\mathrm{b}} R_{\mathrm{m}}\right)$, and propagates back and force through the sample with no absorption $\sigma_{\mathrm{a}}=0$. The transmitted part of flux $F_{\mathrm{b}}^{\mathrm{tr}}$ can be calculated using Eq. 5.4 by replacing $F_{0}$ with $F_{\mathrm{b}} R_{\mathrm{m}}$

$$
F_{\mathrm{b}}^{\mathrm{tr}}=F_{\mathrm{b}} R_{\mathrm{m}} T_{\mathrm{R}}+F_{\mathrm{b}} R_{\mathrm{m}} T_{\mathrm{R}} R_{\mathrm{R}} R_{\mathrm{m}}+F_{\mathrm{b}} R_{\mathrm{m}} T_{\mathrm{R}} R_{\mathrm{R}}^{2} R_{\mathrm{m}}^{2}+\cdots=F_{\mathrm{b}} T \sum_{n=0}^{\infty} R_{\mathrm{R}}^{n} R_{\mathrm{m}}^{n}=\frac{F_{\mathrm{b}} R_{\mathrm{m}} T_{\mathrm{R}}}{1-R_{\mathrm{R}} R_{\mathrm{m}}}
$$

where $T_{\mathrm{R}}$ and $R_{\mathrm{R}}$ are the transmission and reflection of the light at the wavelength of the re-emitted light. Finally we sum all the contributions Eq. 5.4 and Eq. 5.6 for transmitted re-emitted flux

$$
F_{\mathrm{tot}}^{\mathrm{re}-\mathrm{em}}=F_{\mathrm{b}}^{\mathrm{tr}}+F_{\mathrm{T}}^{\mathrm{re}-\mathrm{em}}=\frac{F_{0} T_{\mathrm{R}} R_{\mathrm{r}} R_{\mathrm{m}}}{\left(1-R_{\mathrm{R}} R_{\mathrm{m}}\right)\left(1-R R_{\mathrm{m}}\right)}+\frac{F_{0} T_{\mathrm{r}}}{1-R R_{\mathrm{m}}},
$$

where $F^{\mathrm{re}-\mathrm{em}}$ is the re-emitted light generated on the right side of the slab due to every passage of the excitation light through the slab and $F_{\text {tot }}^{\mathrm{re}-\mathrm{em}}$ is the total flux of the re-emitted light emitted on the right side of the slab. When we remove mirror from the system $\left(R_{\mathrm{m}}=0\right)$ Eq. 5.7 results in

$$
F_{\text {tot }}^{\mathrm{re}-\mathrm{em}}=F_{\mathrm{T}}^{\mathrm{re}-\mathrm{em}}=F_{0} T_{\mathrm{r}}
$$

that is equal to the first passage of $F_{0}$ through the slab. In another extreme when the 


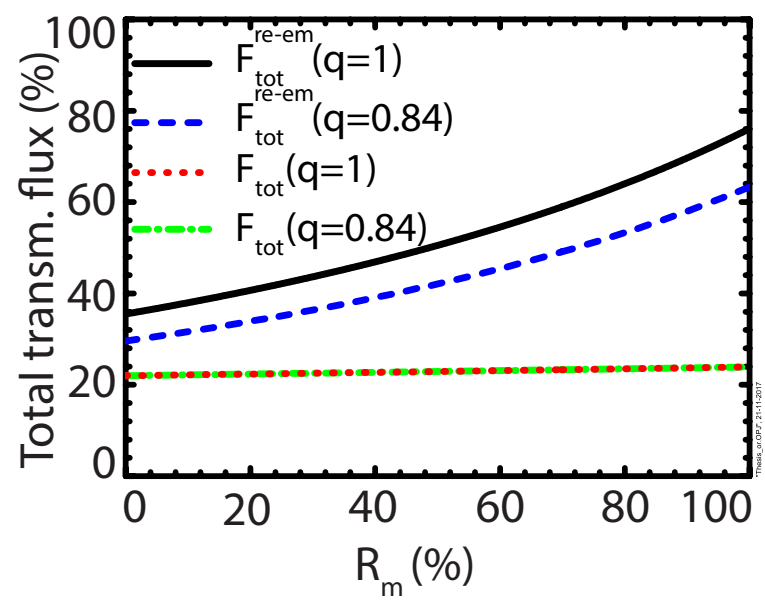

Figure 5.7: Total transmitted flux as a function of mirror reflectivity. Transmitted flux of the scattered and re-emitted light is shown for two values of quantum yield. Total transmitted flux for the re-emitted light depends on the quantum yield. The transmitted flux for the scattered light does not depend on the quantum yield of the phosphor.

mirror is perfect $R_{\mathrm{m}}=1$ and the quantum yield $q=1\left(A=T_{\mathrm{r}}+R_{\mathrm{r}}=1-T-R\right.$, where $A$ is the absorbed light) all incident flux is transmitted in the forward direction

$$
\begin{array}{r}
F_{\mathrm{tot}}+F_{\mathrm{tot}}^{\mathrm{re}-\mathrm{em}}=\frac{F_{0} T}{1-R}+\frac{F_{0} T_{R} R_{\mathrm{r}}}{\left(1-R_{\mathrm{R}}\right)(1-R)}+\frac{F_{0} T_{\mathrm{r}}}{1-R}=\frac{F_{0} T}{1-R}+\frac{F_{0}\left(R_{\mathrm{r}}+T_{\mathrm{r}}\right)}{1-R}= \\
=\frac{F_{0} T}{1-R}++\frac{F_{0}(1-T-R)}{1-R}=F_{0}
\end{array}
$$

where we used the equality $T_{\mathrm{R}}+R_{\mathrm{R}}=1$. This equality follows from the assumption that the re-emitted light is not absorbed by the phosphor as justified in Ch. 4 .

In Fig. 5.7 we plotted Eq. 5.2 and Eq. 5.7 as a function of the mirror reflectivity $R_{\mathrm{m}}$ for two values of the quantum yield $q$. We see that up to $\sim 34 \%$ point of additional re-emitted flux is redirected in the forward direction when a mirror is used in the system $R_{\mathrm{m}}=1$ compared to no mirror $R_{\mathrm{m}}=0$. The re-emitted flux depends considerably on the quantum yield $q$ and mirror reflectivity $R_{\mathrm{m}}$ of the system, while on the contrary, scattered flux does not depend on the quantum yield $q$ and insignificantly depend on the mirror reflectivity $R_{\mathrm{m}}$. This disproportional increase of the re-emitted and scattered flux results in the shift of the color point to the red part of the color triangle. The derived expressions for fluxes can be used for any excitation and emission wavelength $\lambda$, outside the overlap region described in Ch. 3 , 


\subsection{Summary}

We have developed an analytical model that can revolutionize white LED design. It provides a simple, efficient design tool, hence, allowing to access a large design parameter space using an analytical algorithm without adjustable parameters. While our current solution offers a one-dimensional description of the light propagation, the analytical solution of the P3 approximation in 3D geometry 45] is already available and can describe light propagation in complex geometries that are typically employed in white LED design. Additionally, we obtained the expression for the spectrum of a white LED including the back-reflector. Here we derived the expressions for the total flux emitted in the forward direction by model white LED with a reflecting mirror. Our model showed that the use of the mirror provides an increase of the total transmitted flux up to $\sim 34 \%$ point and shifts the color point of the LED to the red part of the spectrum. Our approach will increase the design efficiency by avoiding recurring design efforts and decrease the cost of ownership of white LEDs units for worldwide users. 


\section{Chapter 6}

\section{Universal limit on the applicability of diffusion theory for light propagation}

\subsection{Introduction}

Diffusion theory is one of the most widely used theories for describing physical processes of heat diffusion, electron transport, sound waves and light propagation. It is an approximation to more general transport theory. Diffusion theory for light fail when applied to those special systems in which the scattering is predominantly in the forward direction, or in which absorption is extreme. The precise range of applicability of diffusion theory has never been established. Here we present a fundamental, universal limit beyond which diffusion theory of light gives unphysical results when applied to the scattering materials. In the unphysical range, the errors in transport parameters can reach thousands of percents.

\subsection{Diffusion theory}

Understanding the physics of light in scattering materials with absorption and different degree of anisotropy is crucial in many areas of research, ranging from astrophysics to biology and sustainable energy generation $28,32,40,80,84,103,104]$. The transport theory is used to describe how light propagates in these complex materials [43]. Given the complexity of transport theory, an extremely popular approximation to transport theory is the diffusion theory of light [43].

Diffusion theory has been successfully applied to describe experimental observations on numerous materials with widely varying optical properties such as clouds [105, biological tissue [106, quantum dots [107, powders [108, and white LEDs 97]. Diffusion is supposed 
to be a valid approximation to transport theory for a broad range of transport parameters that characterize scattering media $105,106,109]$. The accepted ranges of validity are typically described by comparing a sample thickness $d$ to several characteristic length scales [77]:

$$
\ell_{\text {tr }} \ll d \ll \ell_{\mathrm{a}},
$$

where the transport mean free path $\ell_{\mathrm{tr}}$ is the average distance light travels before losing information about its initial direction due to scattering, and the absorption mean free path $\ell_{\mathrm{a}}$ is the average distance light travels before being absorbed by $1 / e[23,28,63,92$.

Whereas the inequalities (6.1) provide guidelines for the range of validity, they do only offer a qualitative limit. It has been shown that in some instances the diffusion equation still yields reasonable predictions, although the inequalities (6.1) were violated [110]. Hence, the central questions of our paper are: Is there a sharply defined range of validity of diffusion theory? What happens outside such a possible range? In a nutshell, the answer provided by this chapter are: yes, there is a sharp range of validity, and outside these ranges, we found unphysical behavior.

Both transport theory and diffusion theory describe light using the characteristic scattering length scales $\left(\ell_{\mathrm{s}}, \ell_{\mathrm{a}}, \ell_{\mathrm{tr}}\right)$. The scattering mean free path $\ell_{\mathrm{s}}$ is the average distance light travels between two consecutive collisions. These characteristic length scales have straightforward relations to the single-particle properties $\left(\sigma_{\mathrm{s}}, \sigma_{\mathrm{a}}, \mu\right)$, where $\sigma_{\mathrm{s}}$ is the scattering cross section of the particles constituting the scattering material, $\sigma_{\mathrm{a}}$ where is the absorption scattering cross section of the particles, and $\mu$, is defined as the average scattering angle $\langle\cos \theta\rangle$ of a scattering particle. These relations are 43]

$$
\begin{gathered}
\ell_{\mathrm{s}}=\frac{1}{\rho \sigma_{\mathrm{s}}} \equiv \frac{1}{\mu_{\mathrm{s}}}, \\
\ell_{\mathrm{a}}=\frac{1}{\rho \sigma_{\mathrm{a}}} \equiv \frac{1}{\mu_{a}}, \\
\frac{1}{\ell_{\mathrm{tr}}}=\frac{(1-\mu)}{\ell_{\mathrm{s}}}+\frac{1}{\ell_{\mathrm{a}}} \equiv \frac{1}{\mu_{s}^{\prime}}+\frac{1}{\mu_{a}},
\end{gathered}
$$

where $\rho$ is the density of scattering particles. The introduced coefficients $\mu_{s}, \mu_{a}, \mu_{s}^{\prime}$ are often used in biological tissues studies 106, 111, 112. The Eqs. (6.2,6.4 are essentially the result of expansions of scattering theory in inverse density $1 / \rho$, and their validity goes far beyond transport theory 43 .

In widely-used geometries, such as a finite slab or a semi-infinite slab, the diffusion 


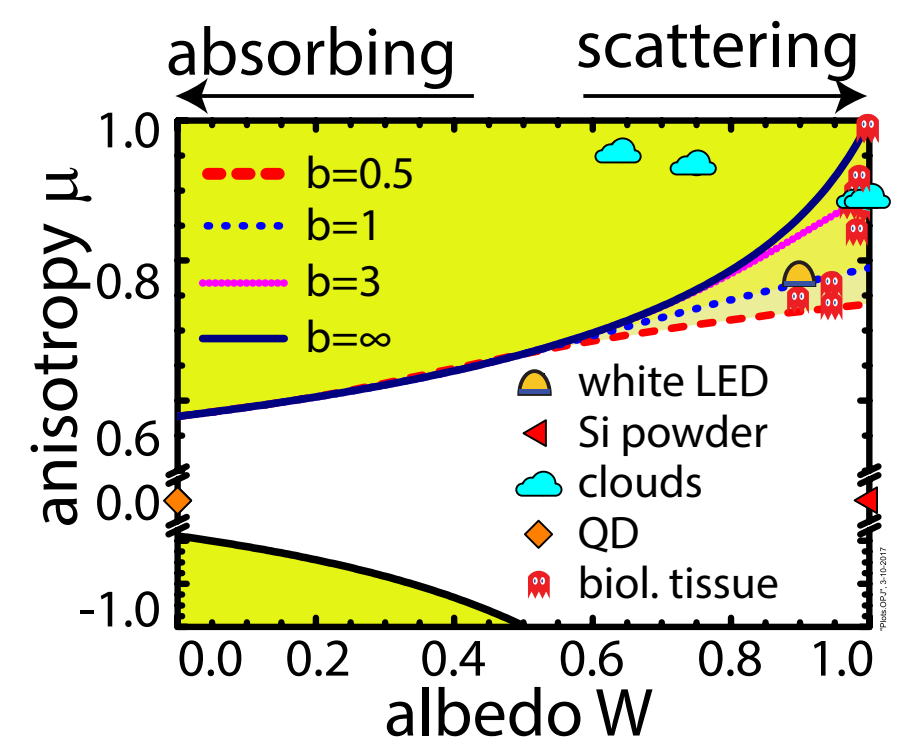

Figure 6.1: Validity range of diffusion theory for a slab shown as a 2D map. Lime-green regions are the areas where the diffusion equation gives unphysical results. The dashed and dotted curves show the boundary of validity for different values of $b$. The solid lines indicate the boundary for a semi-infinite slab $(b=\infty)$. The data symbols represent sample parameters for popular scattering media, as described in the legend [66, 97, 105 107].

equation allows for simple, analytical solutions. For instance, for a plane wave incident upon a finite slab with thickness $d$ the diffusion equation for the diffuse energy density $U_{\mathrm{d}}(z)$ has the form 43

$$
\frac{\partial^{2}}{\partial z^{2}} U_{\mathrm{d}}(z)-\kappa_{\mathrm{d}}^{2} U_{\mathrm{d}}(z)=-Q_{0} \exp \left(-\rho \sigma_{\mathrm{t}} z\right)
$$

where $Q_{0}=\left[3 \rho \sigma_{\mathrm{s}} \rho \sigma_{\mathrm{tr}}+3 \rho \sigma_{\mathrm{s}} \rho \sigma_{\mathrm{t}} \mu\right]\left(F_{0} / 4 \pi\right), F_{0}$ is the magnitude of the incident flux, $\sigma_{\mathrm{tr}}=$ $(1-\mu) \sigma_{\mathrm{s}}+\sigma_{\mathrm{a}}$ is the transport cross section, and $\kappa_{\mathrm{d}}^{2}=3 \sigma_{\mathrm{a}} \sigma_{\mathrm{tr}}$. The mixed boundary conditions are

$$
\begin{array}{ll}
U_{\mathrm{d}}(z)-h \frac{\partial}{\partial z} U_{\mathrm{d}}(z)+\frac{Q_{1}(z)}{2 \pi}=0 & \text { at } z=0, \\
U_{\mathrm{d}}(z)+h \frac{\partial}{\partial z} U_{\mathrm{d}}(z)-\frac{Q_{1}(z)}{2 \pi}=0 & \text { at } z=d,
\end{array}
$$

where $Q_{1}(z)=\sigma_{\mathrm{s}} \mu \sigma_{\mathrm{tr}} F_{0} \exp \left(-\rho \sigma_{\mathrm{t}} z\right), \sigma_{\mathrm{t}} \equiv \sigma_{\mathrm{s}}+\sigma_{\mathrm{a}}$ is the total cross section, and $h \equiv$ $2 /\left(3 \rho \sigma_{\mathrm{tr}}\right)$. The solution to differential equation Eq. 6.5 with boundary conditions 6.6. 6.7) is textbook material [43. As this solution is the starting point of our discussion, we have verified this solution both analytically and numerically. The diffuse energy density 
$U_{\mathrm{d}}(z)$ is a scalar, positive definite quantity rigorously expressed as

$$
U_{\mathrm{d}}(z) \geq 0 \quad \forall z \in[0, d]
$$

The flux $\boldsymbol{F}(z) \equiv F(z) \hat{\boldsymbol{z}}$ is a one-dimensional vector [43], whose direction, changes from leftward at the front surface to rightwards at the back surface of the slab, expressed rigorously as

$$
\begin{aligned}
& F(z)<0 \quad \text { at } z=0, \\
& F(z)>0 \quad \text { at } z=d,
\end{aligned}
$$

the total diffuse flux directed inwards must be zero. The rigorous conditions 6.8 6.10 replace the inequalities (6.1). When any of these conditions 6.86 .10 is violated the diffusion equation fails, as experimentally observable quantities would take on unphysical values, such as negative reflection or transmission coefficients, or a negative energy density.

To check the validity of the diffusion equation for the slab and the semi-infinite slab we explored the full parameter space. Several independent sets can fully characterize the sample. We have chosen the set consisting of the three dimensionless parameters $(W, \mu, b)$, where the albedo $W$ is defined as $W \equiv \sigma_{\mathrm{s}} /\left(\sigma_{\mathrm{s}}+\sigma_{\mathrm{a}}\right)$, and where the dimensionless optical thickness of the slab $b$ is defined as $b \equiv d / \ell_{\mathrm{s}}$.

\subsection{Results}

Our main results are summarized in Fig. 6.1. The two lime-green regions indicate that part of the parameter space where diffusion theory fails, as it generates negative energy density $U_{\mathrm{d}}(z)$. We discovered that the conditions on the flux $F(z)$ and the energy density $U(z)$ are equivalent. In Fig. 6.1 the two yellow-green boundaries of the applicability range of the diffusion theory were calculated from the following expressions:

$$
\begin{aligned}
& U_{\mathrm{d}}(0)=0, \quad \text { at the right side of the slab, } \\
& U_{\mathrm{d}}(d)=0, \quad \text { at the left side of the slab. }
\end{aligned}
$$

The equivalent conditions for the fluxes are formulated as follows:

$$
\begin{aligned}
& F(0)=0, \quad \text { at the right side of the slab, } \\
& F(d)=0, \quad \text { at the left side of the slab. }
\end{aligned}
$$


Naively, one would expect the boundaries defining the validity of the diffusion equation to depend on the optical density. However, we find that only the top region depends significantly on the optical thickness, as shown in Fig. 6.1. When the albedo $W>0.5$, the colored area of the top region is more significant for the greater optical thickness $b$. When $W<0.5$, we observe no changes in the top colored area for different optical thicknesses $b$. This insensitivity for strongly absorbing samples can be explained by the fact that light never reaches the backward boundary due to the strong absorption.

We explore some limiting cases relevant for experiments. In case of systems without absorption $(W=1)$ Eqs. 6.11 6.12 simplified to the following condition on the anisotropy parameter $\mu$ for the diffusion equation to have physical solutions

$$
\mu \leq \frac{-1+3 b+\exp (-b)}{3 b}
$$

In the additional limit of vanishing optical thickness we obtain

$$
\mu \leq \frac{2}{3} \quad \text { at } b \rightarrow 0, W=1
$$

for the diffusion approximation to hold. The limit $b \rightarrow \infty$ and $W=1$ gives physical results for all values of $\mu$. A slab with optical thickness $b>10$ can for all practical purposes be considered to behave as a semi-infinite slab.

From an experimental point of view, a scattering sample is typically characterized with the set of length scales $\left(d, \ell_{\mathrm{tr}}, L_{\mathrm{a}}\right)$, where the absorption length $L_{\mathrm{a}}$ is the average distance between the start and end of a random-walk with absorption mean free path $\ell_{\mathrm{a}}$. We emphasize that the set $\left(d, \ell_{\mathrm{tr}}, L_{\mathrm{a}}\right)$ will always be a valid set characterizing light transport through a sample, irrespective of the validity of diffusion theory.

\subsection{Error map of the diffusion theory}

It is an experimentally relevant question how scientists who study samples that lie in the unphysical according to Fig. 6.1 regions of diffusion theory yet still interpreting the results with diffusion theory without encountering any problem. How is this possible? In a typical experiment, the total transmission coefficient $T$ and total reflection coefficient $R$ are measured. From these experimental data, the lengths $\left(\ell_{\mathrm{tr}}^{\mathrm{d}}, L_{\mathrm{a}}^{\mathrm{d}}\right)$ are inferred using diffusion theory, and no unphysical behavior is found for samples having $(b, W, \mu)$ far in the unphysical regions. In such a case the extracted values $\left(\ell_{\mathrm{tr}}^{\mathrm{d}}, L_{\mathrm{a}}^{\mathrm{d}}\right)$ correspond to values for $(b, W, \mu)$ that differ strongly from the true values $(b, W, \mu)$ for those samples. We will now investigate 


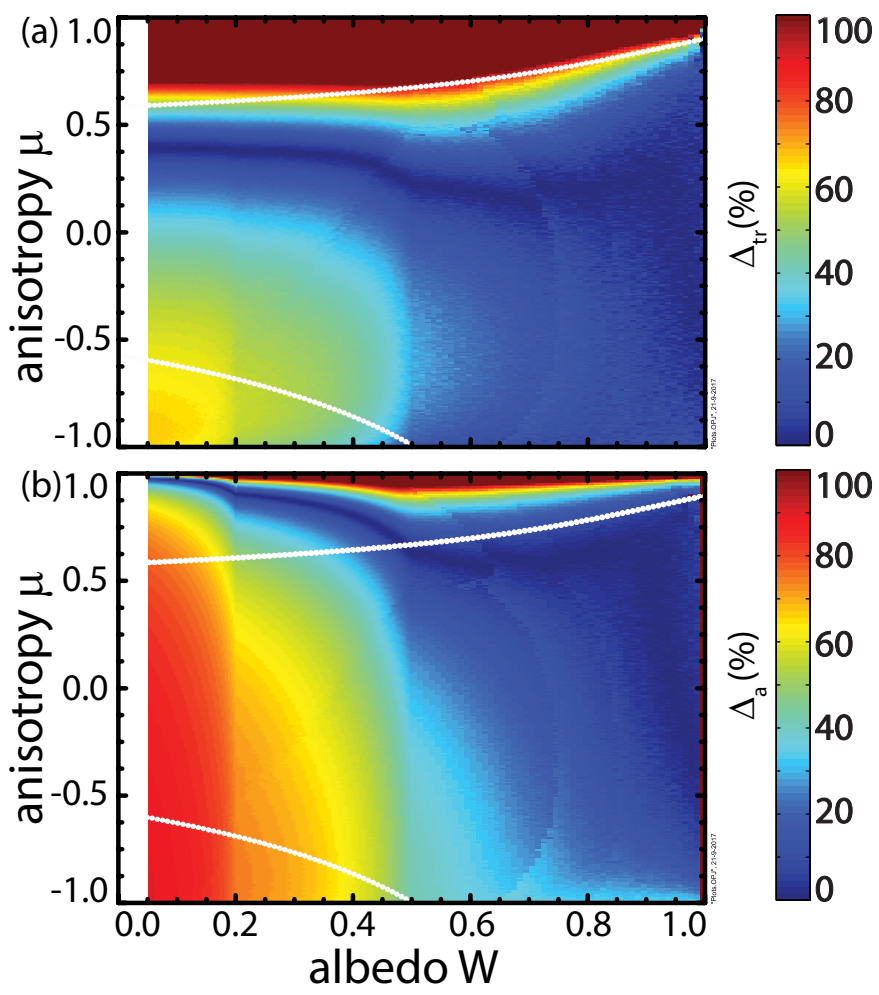

Figure 6.2: Relative error in diffusion theory calculations shown as a 2D map. The white curves show the analytically derived boundary of the diffusion theory validity range for the optical thickness $b=3$. (a) Color gradient indicates the relative error $\Delta_{\text {tr }}$ in the obtained mean free path $\ell_{\mathrm{tr}}^{d}$ using the diffusion theory compared to the rigorous $\ell_{\mathrm{tr}}$. (b) Color gradient indicates the relative error $\Delta_{\mathrm{a}}$ in the obtained absorption length $\ell_{\mathrm{a}}^{d}$ using the diffusion theory compared to the rigorous $\ell_{\mathrm{a}}$.

how much they differ.

To gauge the errors associated with the diffusion approximation we have performed extensive Monte Carlo simulations. The simulation procedure is described in details in Ref. [97]. For the simulations samples are characterized by the set $\left(b, \ell_{\mathrm{tr}}, L_{\mathrm{a}}\right)$. For several values of $b$ we calculate the transmission $T$ and the reflection $R$ for many values of the pair $(W, \mu)$ and convert this set onto the $\left(\ell_{\mathrm{tr}}, L_{\mathrm{a}}\right)$ space. So we have an exact mapping of $\left(\ell_{\mathrm{tr}}, L_{\mathrm{a}}\right)$ to $(T, R)$ and vice versa for a specific value of $b$. Given the set $(T, R)$ obtained from our simulations we can calculate with diffusion theory values for the length scales: $\left(\ell_{\mathrm{tr}}^{\mathrm{d}}, L_{\mathrm{a}}^{\mathrm{d}}\right)$ that would produce these values for $T$ and $R$. These approximate values should be compared with the rigorous values $\left(\ell_{\mathrm{tr}}, L_{\mathrm{a}}\right)$.

To evaluate the relative error of the length scales obtained with diffusion theory we define relative errors

$$
\Delta_{\mathrm{tr}} \equiv \frac{\left|\ell_{\mathrm{tr}}-\ell_{\mathrm{tr}}^{\mathrm{d}}\right|}{\ell_{\mathrm{tr}}^{\mathrm{d}}}
$$




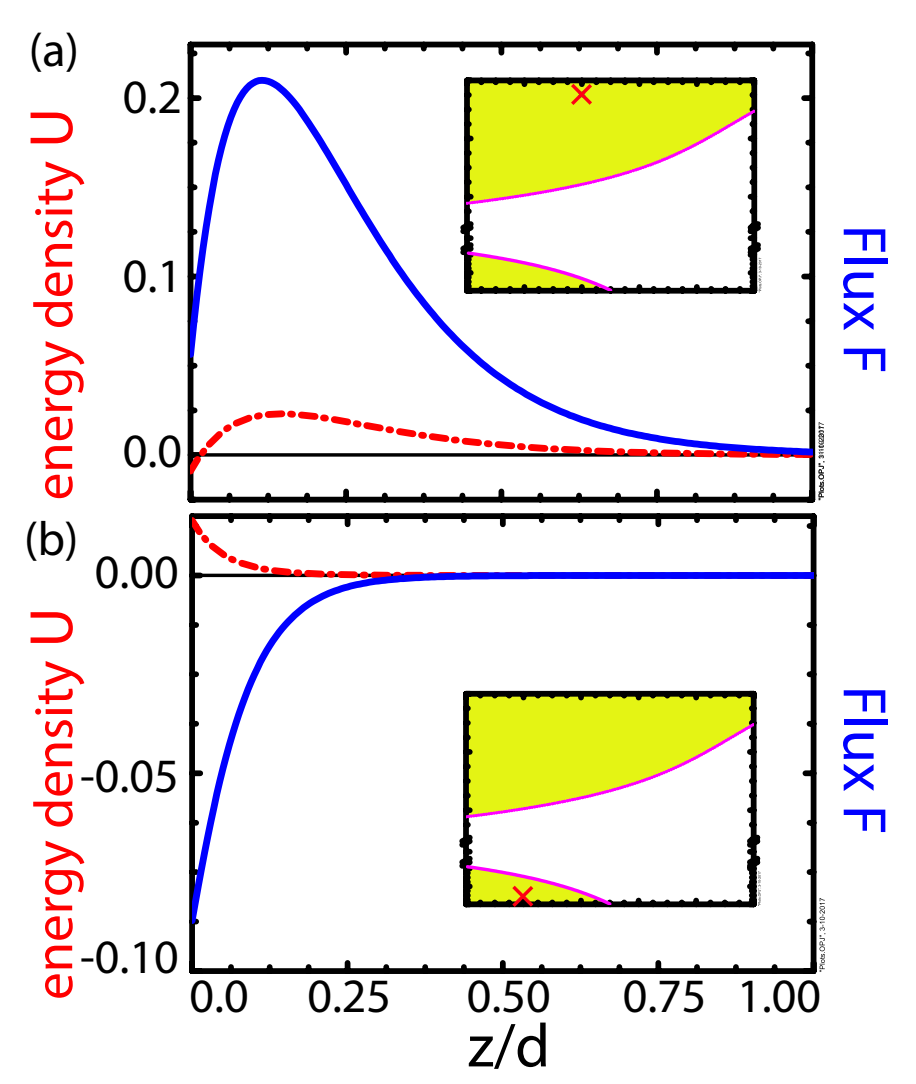

Figure 6.3: Energy density (red curves) and flux (blue curves) in the unphysical range of transport parameters. (a) Example of a strongly forward scattering and absorbing material where the unphysical conditions on the left boundary of the slab $F(0)>0$ and $U(0)<0$ hold. (b) Example of a strongly backward scattering material with strong absorption where the unphysical conditions at the right boundary of the slab $F(d)<0$ and $U(d)<0$ hold. Insets in both graphs are similar to Fig. 6.1. Red crosses indicate parameters of the samples in (a) and (b). Results are shown for $b=3$. Energy density is scaled with $F_{0} / 4 \pi$, and the flux is scaled with $F_{0}, F_{0}$ being the magnitude of the incident flux.

and

$$
\Delta_{\mathrm{a}} \equiv \frac{\left|L_{\mathrm{a}}-L_{\mathrm{a}}^{\mathrm{d}}\right|}{L_{\mathrm{a}}^{\mathrm{d}}} .
$$

In Fig. 6.2 we show the $2 \mathrm{D}$ map of the relative errors for $\mathrm{b}=3$ for both $\Delta_{\operatorname{tr}}$ and $\Delta_{\mathrm{a}}$. First, we consider the range of albedo $W \geq 0.5$. For this albedo range the relative errors $\Delta_{\text {tr }}$ and $\Delta_{\mathrm{a}}$ do not exceed $10 \%$ inside the major part of the physical area. In the narrow range close to the analytical boundary relative errors for the $\ell_{\operatorname{tr}}$ can reach up to $80 \%$ for low albedo $W$ in the physical region. The relative error for $\ell_{\mathrm{a}}$ is less then $10 \%$ near the analytical boundary, but shows higher error up to $40 \%$ near the $\mu=-1$. For $W=1$ we see a narrow red region for all values of $\mu$ in Fig. 6.2. Diffusion theory with absorption always erroneously predict $\left(\ell_{\mathrm{tr}}^{\mathrm{d}}, L_{\mathrm{a}}^{\mathrm{d}}\right)$ for $W=1$. Inside the unphysical region, the relative error $\Delta_{\mathrm{tr}}$ 
exceeds $100 \%$ and $\Delta_{\mathrm{a}}$ exceeds $10 \%$ for the albedo mentioned before.

Secondly we consider the albedo range of $W \leq 0.5$, where the inequality (6.1) is violated. In this region the diffusion theory results in relative errors larger than $50 \%$, except for a narrow (blue) island that can be seen in the left part of Fig. 6.2. (a) and an island in Fig. 6.2(a). The magnitude of errors in these two regions does not exceed 20\%. These two islands do not overlap, meaning that if we identify one parameter from the pair $\left(\ell_{\mathrm{tr}}^{\mathrm{d}}, L_{\mathrm{a}}^{\mathrm{d}}\right)$ correctly, the corresponding another parameter will have errors exceeding 50\%. Without any prior knowledge about the sample, we cannot guess what quantity from the pair $\left(\ell_{\mathrm{tr}}^{\mathrm{d}}, L_{\mathrm{a}}^{\mathrm{d}}\right)$ is extracted correctly. To conclude, diffusion theory gives unreliable results when interpreting data on samples with $W \leq 0.5$, because the error of transport parameters can reach thousands of percent.

In Fig. 6.2 we have indicated with white lines the analytical boundaries of the validity of the diffusion theory, for the case of $b=3$. These boundaries divide the scattering parameter space into three parts. The diffusion theory results in physical solutions only in the middle region. For $W \geq 0.5$ the white line separates the area with the high error from the part with low error obtained with Monte Carlo simulations. For $W \leq 0.5$ diffusion theory results in significant errors as discussed before.

To indicate more in detail the wrong predictions of the diffusion theory from the invalid region, we show in Fig. 6.3 the energy density and flux for two samples with correct scattering parameters. Transport parameters of sample 1 and sample 2 are shown in the inset of Fig. 6.3(a)-(b) respectively. In Fig. 6.3(a) the flux and energy density show unphysical behavior near the left boundary of the sample. For the transport parameters in Fig. 6.3(b) the flux and energy density show unphysical behavior near the right boundary of the sample. The relative errors of the length scales are $\left(\Delta_{\operatorname{tr}}=1056 \%, \Delta_{\mathrm{a}}=115 \%\right)$ and $\left(\Delta_{\mathrm{tr}}=56 \%, \Delta_{\mathrm{a}}=74 \%\right)$ for sample 1 and 2 respectively.

\subsection{Summary}

In conclusion, we have derived the fundamental limit of diffusion theory in media with absorption and anisotropic scattering. We identified a set of three parameters consisting of the anisotropy factor, the albedo, and the optical thickness that together fully characterizes any multiply scattering material with or without absorption. It is pointed out that in the space defined by this parameter set there are large areas where the diffusion equation gives unphysical results, such as negative energy densities. We demonstrated that when diffusion theory is used to infer mean free paths and absorption lengths from experimental data, 
relative errors in these length scales can be as large as thousands of $\%$. 


\section{Chapter 7}

\section{Reversible tuning of the color point of a white LED}

\subsection{Introduction}

White light is conveniently characterized by a color point that is represented on the color space shown in Fig. 7.1 (see Appendix). The color point of a white LED depends on its design parameters (see Ch. 5). For example in Fig. 7.1 we show how the color point depends on the phosphor particle concentration. When the design parameters are chosen, the color point of the white LED cannot be changed. Here, we show that wavefront shaping technique can be employed to reversibly tune the color point of a white LED without changing the design parameters (see Fig. 7.1).

\subsection{Wavefront shaping}

Wavefront shaping defines how the light travels through the scattering medium. It exploits the interference effects inside the scattering medium in such a way that the scattering medium can even be transformed into a lens [22, 113, 114]. The plane wave that is incident on the scattering medium is diffused while undergoing multiple scattering inside the slab in the absence of wavefront shaping. However, when we change the phase or the amplitude of the incoming wavefront with the help of wavefront shaping, the light can be focused into the single focus spot behind the sample (see Fig. 7.2). To achieve the focusing effect, the incident wavefront is optimized using algorithms described in detail in Ref. 115. The quality of the wavefront is gauged using the enhancement $\eta$, which is defined as

$$
\eta \equiv \frac{F_{\text {opt }}(\lambda)}{<F_{\text {rand }}(\lambda)>},
$$




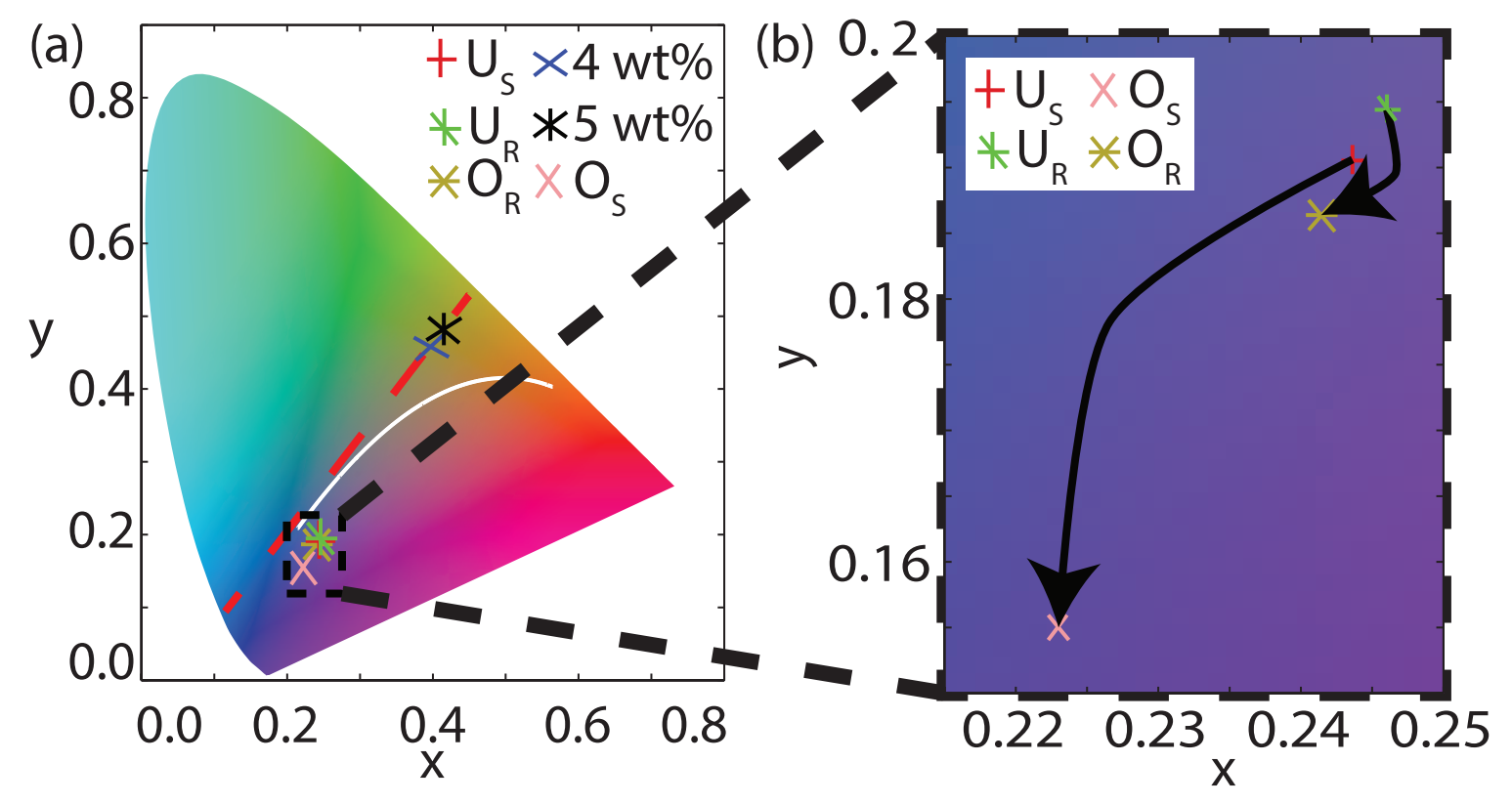

Figure 7.1: Tuning of the color point of a white LED. (a) Color triangle with the color points obtained under different conditions. The dashed red line represents the color point as a function of concentration for the given phosphor. Red crosses correspond to the color point of the scattered light before and after wavefront optimization. Green crosses correspond to the color point of the re-emitted light before and after optimization. Black and dark blue crosses correspond to the color point of the diffuser plates with different phosphor concentrations [97]. (b) The dashed inset shows enlarged area with the color points extracted from the experiment.

where $F_{\text {opt }}(\lambda)$ is the flux of the diffused light with an optimized wavefront and $\left\langle F_{\text {rand }}(\lambda)\right\rangle$ is the flux of the diffused light with an unoptimized or random wavefront.

Here we exploit wavefront shaping to change the color point of a white LED reversibly. There are two main differences between the wavefront shaping approach described above and one that is used in this work. The first difference is that the wavefront shaping is done here on a large area of the order of $\mathrm{mm}$. We measure all outgoing light from the sample. The second difference is that the phosphor particles that are used in white LEDs not only scatter light but also absorb and re-emit part of it in the red part of the spectrum. Hence, the optimization can be done for the scattered light and re-emitted light. Here we optimize either on the scattered light or the re-emitted light.

Having these two differences in mind, we define two enhancements, one for the scattered light $\left(\eta_{s}\right)$ and one for the re-emitted light $\left(\eta_{\mathrm{r}}\right)$

$$
\eta_{s, r} \equiv \frac{\int_{\lambda_{1}}^{\lambda_{2}} F_{\mathrm{opt}}(\lambda) d \lambda}{\int_{\lambda_{1}}^{\lambda_{2}}<F_{\text {rand }}(\lambda)>d \lambda},
$$


(a)

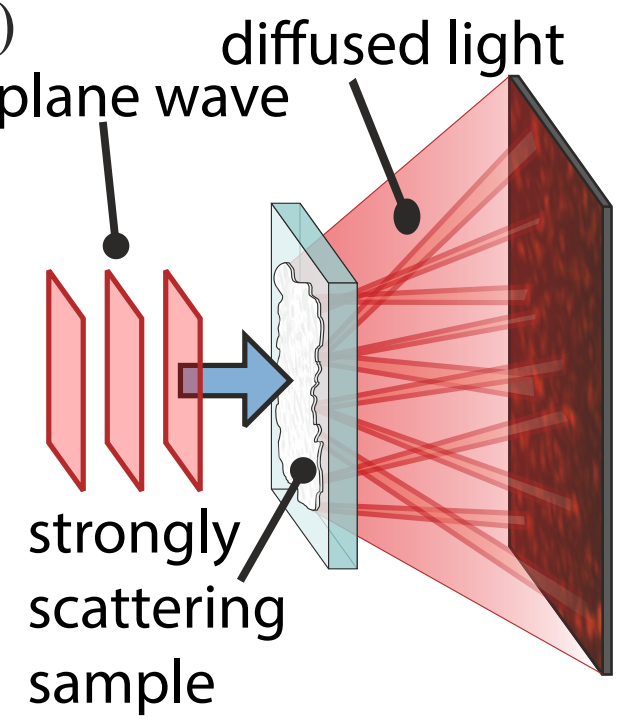

(b) focused light shaped wave

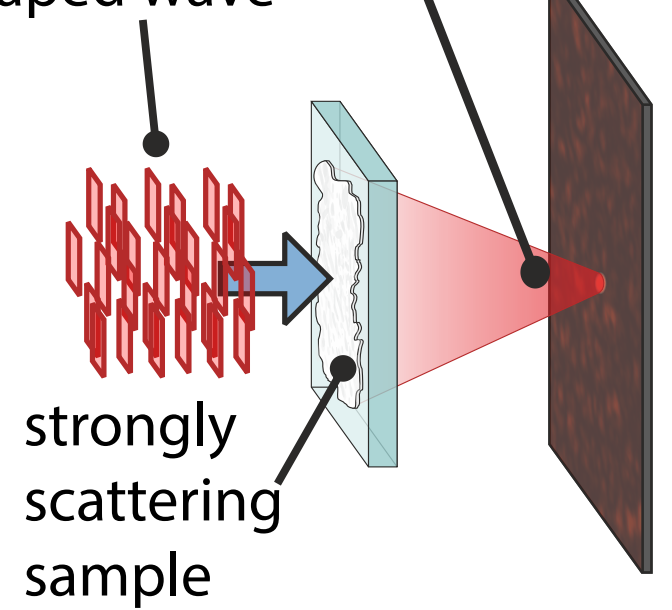

Figure 7.2: Wavefront shaping technique. (a) A plane wave is incident on a scattering medium. The light is multiply scattered resulting in the transmitted diffuse light. (b) By applying a certain wavefront, the light is scattered in the medium in such a way that it focuses behind the scattering medium. Figure adopted from 116 .

where $F_{\text {opt }}(\lambda)$ is the flux at wavelength $\lambda$ integrated over the area of the diffused spot when an optimized wavefront is used, $F_{\text {rand }}(\lambda)$ is the flux at wavelength $\lambda$ integrated over the area of the diffused spot when an unoptimized wavefront is used, and $\langle\cdots\rangle$ denotes the average over many random wavefronts. The integration is performed for the following range of wavelengths: for the scattered light we use $\left(\lambda_{1}=455 \mathrm{~nm}, \lambda_{2}=460 \mathrm{~nm}\right)$ and for the re-emitted light we use $\left(\lambda_{1}=475 \mathrm{~nm}, \lambda_{2}=700 \mathrm{~nm}\right.$ ) (see Fig 7.8).

The expected enhancement can be theoretically estimated for a multiple channel optimization in the following way 117:

$$
\eta=\frac{\pi}{4} \frac{N-1}{M}+1
$$

where $\mathrm{N}$ is the number of segments of the spatial light modulator (SLM), $\mathrm{M}$ is the number of modes in the system that depends notably on the wavelength, and the bandwidth of the light source. The number of modes $M_{\mathrm{s}}$ for the scattered light can be estimated to be $M_{\mathrm{s}}=2 \pi A \arcsin (\mathrm{NA}) / \lambda^{2}$, where $A$ is the area of the diffused spot, NA is the numerical aperture of the setup, and $\lambda$ is the illumination wavelength [117]. The number of modes $M_{\mathrm{r}}$ for the re-emitted light is estimated as $M_{\mathrm{r}}=8 \pi n_{\mathrm{eff}}^{3} V \Delta \lambda / \lambda^{4}$, where $V$ is the volume of the diffused light cone inside the sample [118], $\Delta \lambda=\lambda_{2}-\lambda_{1}=225 \mathrm{~nm}$ is the full width at half maximum of the emission spectrum, $\lambda=587.5 \mathrm{~nm}$ is the central wavelength, and $n_{\text {eff }}$ 


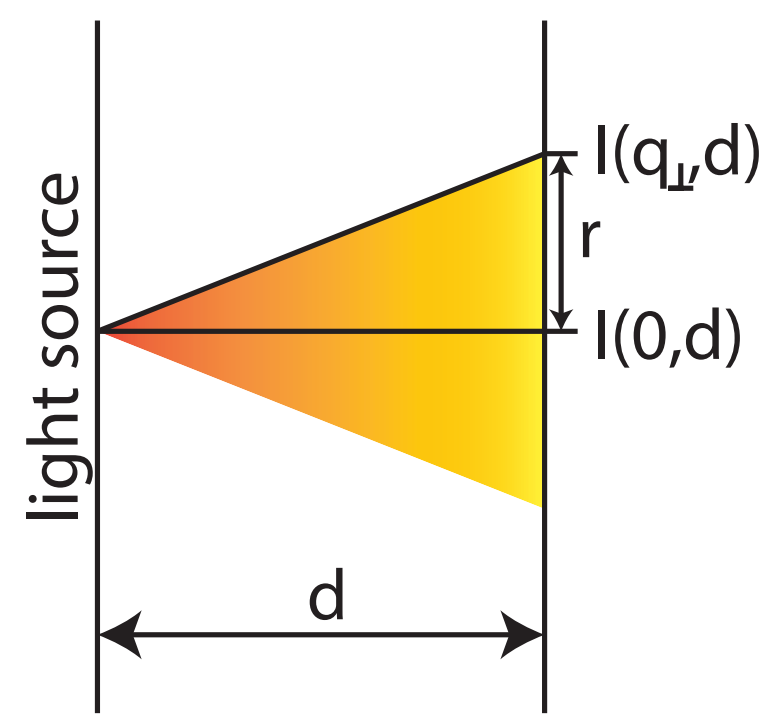

Figure 7.3: Schematic of the diffused light cone inside a slab of scattering material. The focused light creates point source on the left surface of the plate. Light diffuses inside the scattering medium, and forms the diffuse light spot with radius $r$ on the right surface of the slab.

is the effective refractive index.

To calculate the number of modes $M$ we need to estimate the size of the diffused spot created on the sample by the incident beam. To estimate the size of the diffused spot we use the expression for the diffuse flux using the $2 \mathrm{D}$ diffusion equation in Fourier $k$-space [117

$$
F_{\mathrm{d}}\left(\mathbf{k}_{\perp}, \mathrm{z}\right)=A \frac{\sinh \left(k_{\perp}\left[d_{e}-z-z_{\mathrm{e}}\right]\right) \sinh \left(k_{\perp}\left[\ell_{\mathrm{tr}}+z_{\mathrm{e}}\right]\right)}{k_{\perp} \sinh \left(k_{\perp} d_{e}\right)}
$$

where $A$ is a constant that depends on the incident flux and on the diffusion constant, $d_{e} \equiv d+2 z_{e}, k_{\perp} \equiv \sqrt{k_{\perp \mathrm{x}}^{2}+k_{\perp \mathrm{y}}^{2}}$ and $z_{\mathrm{e}}=\mathrm{J}_{1} \ell_{\mathrm{tr}} / \mathrm{J}_{0}$, the constants $\mathrm{J}_{0}$ and $\mathrm{J}_{1}$ are defined in Ch. 2 Eq. 2.91. We define the radius of the diffused spot to be the solution of the following equation (see Fig. 7.3)

$$
\frac{F_{\mathrm{d}}\left(k_{\perp}, \mathrm{d}\right)}{F_{\mathrm{d}}(0, \mathrm{~d})}=e^{-2}
$$

Since Eq. 7.5 is a transcendent equation, it cannot be solved exactly. For the diffuser plate used in the experiment the numerical solution of Eq. 7.5 yields $k_{\perp}=r=1.28 \mathrm{~mm}$. From here we calculate the area of the diffused spot $A=\pi r^{2}=5 \mathrm{~mm}^{2}$, and the number of modes of the scattered light to be $M_{\mathrm{s}}=10^{8}$. The mode volume of the re-emitted light is calculated to be $V=\pi r^{2} d / 3=3.43 \mathrm{~mm}^{3}$, so the number of modes for the re-emitted light is huge: $M_{\mathrm{r}}=4.3 \times 10^{11}$. Typically in wavefront shaping experiments, the number of modes is of the order of $10^{3}$ for the scattered light, which allows gaining substantial control over 
light in the experiment.

\subsection{Setup}

In this chapter, we discuss the setup based on a setup that was constructed to demonstrate wavefront shaping to a broad audience robustly. Hence parameters of the setup were optimized for demonstration purposes. The experimental setup is shown in Fig. 7.4. The light source is a blue diode laser (Roithener Lasertechnik, rldd450-40-5) with a wavelength $\lambda=457 \mathrm{~nm}$ to excite the phosphor. The beam is expanded to fill the active area of the digital micromirror device (DMD). The original beam shape of the laser is elliptical with dimensions 3 by $5 \mathrm{~mm}^{2}$. The beam is expanded by 6.7 times in the horizontal direction using two plano-convex cylindrical lenses $(\mathrm{C} 1$ and $\mathrm{C} 2)$ to illuminate a higher number of segments on the DMD. Mirror M1 and M2 direct the beam onto a DMD. The DMD (Vialux V-7001, DLP700) has the resolution of $1024 \times 786$ pixels and a pixel size of $13.68 \mu \mathrm{m}$. The angle of incidence of light on the DMD should be set to 24 degrees to control the pixels of the DMD. The on-position of the pixel corresponds to the micromirror position that reflects light in the direction of lens L1, and off-position of the pixel corresponds to the location of the micromirror that reflects light to the beam dump (CB). We apply the superpixel method 119 using lenses L1, L2 and the aperture (A). The lenses both have a focal length $150 \mathrm{~mm}$ and are placed $300 \mathrm{~mm}$ apart in a $4 \mathrm{f}$ configuration. In the focal plane of L1, the aperture (A) blocks the first diffraction order. Mirrors M3 and M4 are used to steer the beam. The beam is focused by a $75 \mathrm{~mm}$ lens (L3) onto the sample (S). The sample is silicone (PDMS) plate with phosphor particles (YAG:Ce ${ }^{3+}$ ) with a concentration of YAG: $\mathrm{Ce}^{3+}$ particles of 4 wt \% made by Jan Jansen at Philips Lighting. The sample has a circular shape with the diameter of $6 \mathrm{~cm}$ and is $2 \mathrm{~mm}$ thick. The optical characterization of the sample is described in detail in Chapter 4.

The sample is placed in the focal plane of the lens L3. A 2-inch lens L4 with focal length $35 \mathrm{~mm}$ is positioned at a distance of $39 \mathrm{~mm}$ from the sample. The beam is split using a 92/8 pellicle beam splitter. The feedback arm receives $8 \%$ of the light and $9 \%$ of the light is sent to the spectrometer arm. In the feedback arm, the light is spectrally filtered using a shortpass (FES0500) or long-pass (FEL0500-1V1-45-11) filter F to optimize on the scattered or the re-emitted light respectively. The cutoff wavelength of the filter is $\lambda=500 \mathrm{~nm}$. An image of the diffused spot on the sample is projected on the charged coupled device camera (CCD, Allied Vision Guppy PRO F-146) placed at distance $320 \mathrm{~mm}$ from L4 (01LAGO13). A feedback system FB processes the images taken by the CCD and sent it to the DMD. In 


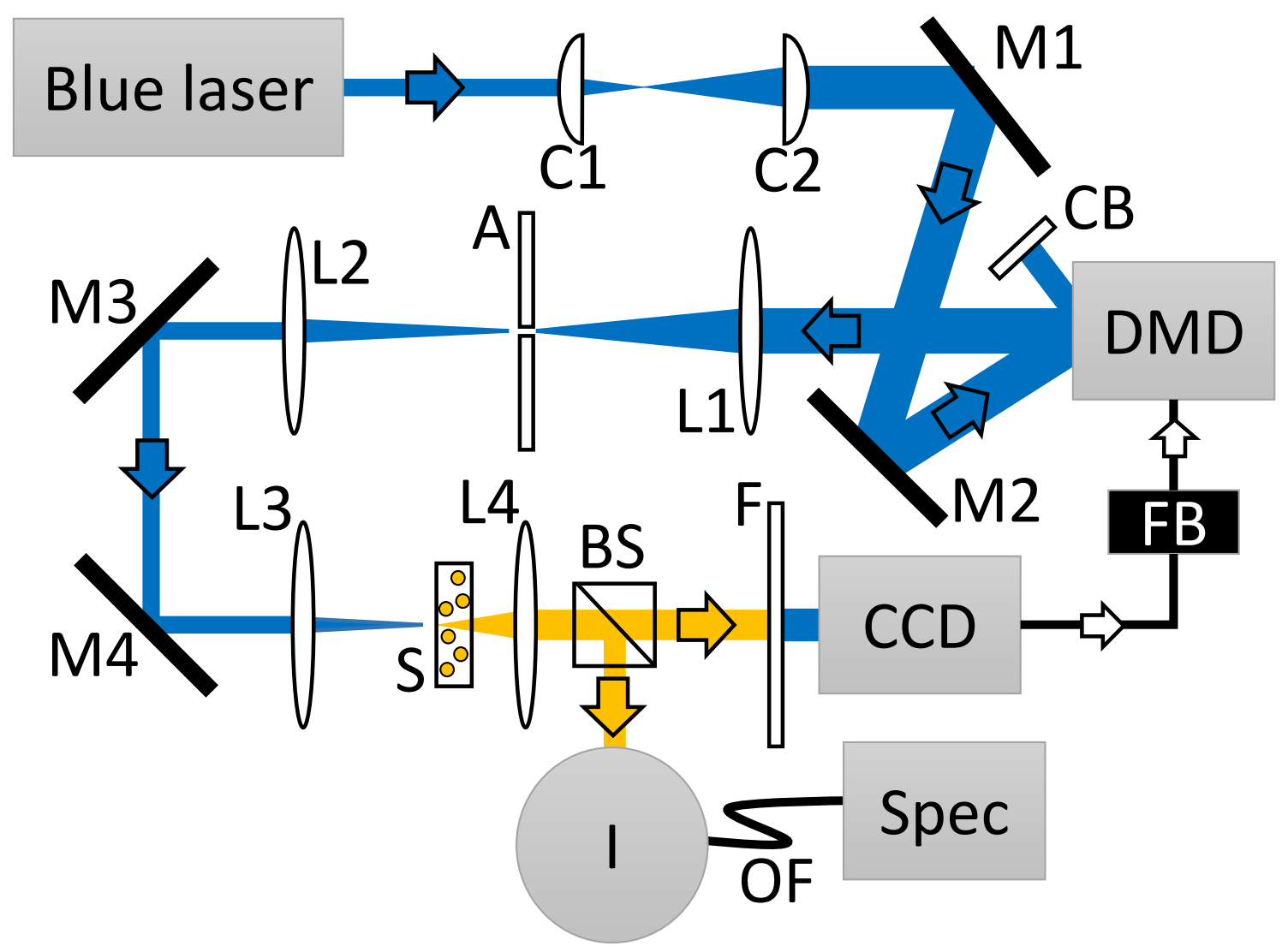

Figure 7.4: A schematic of the setup for wavefront shaping on a phosphor sample. The blue laser beam is expanded and modulated by the digital mirror device (DMD). With a series of lenses and mirrors, the beam is focused onto the sample surface. The flux is transmitted through the sample and collected by the lens L4, and split by the beam splitter between CCD camera and integrating sphere.

the spectrometer arm, an integrating sphere $\mathrm{I}$ is placed at the same distance $(320 \mathrm{~mm})$ to L4 as the CCD. In this way, we ensure that the collected flux is the same for the CCD and the integrating sphere. The spectrum of light is detected by a spectrometer (Spec, Avantes AvaSpec-ULS2048XL-EVO) connected to the integrating sphere through the optical fiber OF. The setup allows to perform wavefront shaping optimization and acquire the spectrum of emitted light simultaneously.

The feedback system is controlled by a LabView program that optimizes a wavefront using the partitioning algorithm [115]. The optimization procedure starts by applying a random wavefront to the DMD and taking an image of the speckle pattern. Then $50 \%$ of the segments are randomly selected and shifted 3 times with the phase shift $\pi / 4$. Generated wavefront is sent back to the DMD, and a new image is acquired with the CCD. This process is repeated over and over again until the desired wavefront is obtained. The optimization 


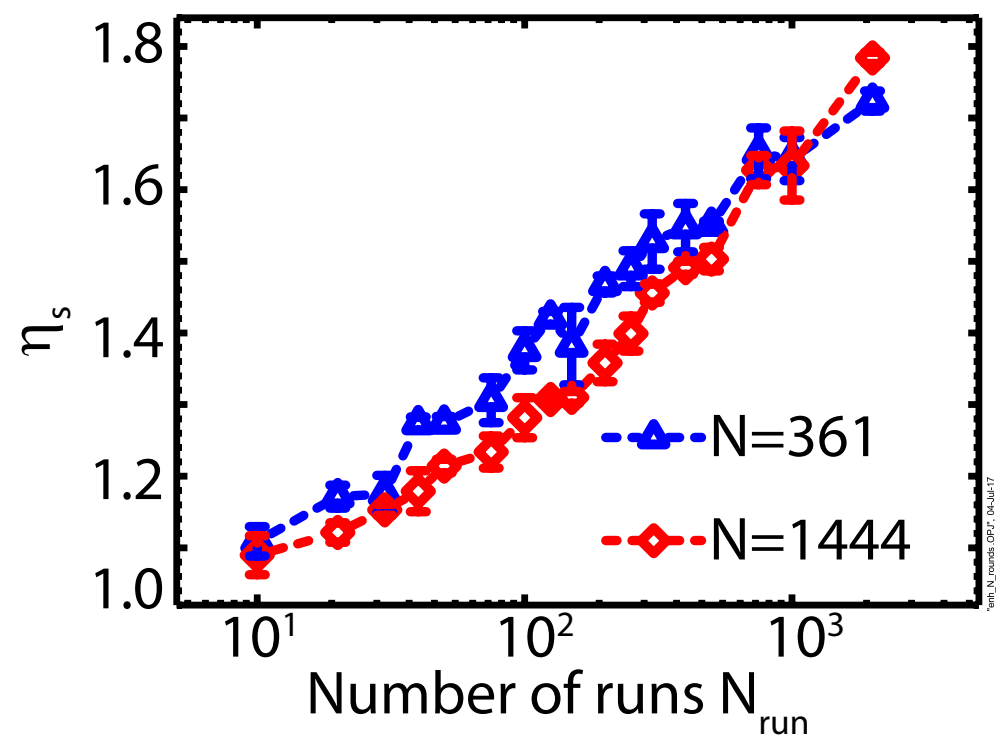

Figure 7.5: The wavefront enhancement registered on the CCD as a function of the number of optimization rounds $N_{\text {run }}$. Each data point is averaged over 3 measurement rounds; the error bars are the standard deviations. The number of segments used during the optimization is indicated in the legend.

process takes approximately 30 min to achieve enhancement of 2 .

\subsection{Characterization of the setup}

\subsubsection{Optimization algorithm}

For optimal performance of the optimization algorithm, we need to identify the number of segments $N$ used on the DMD, and the number of optimization runs $N_{\text {run. }}$. Here we find experimentally the number of runs required for optimization. The enhancement $\eta$ on the CCD is measured as a function of $N_{\text {run }}$ for two different values of total controlled segments $(N=361$ or 1444$)$. The segments are arranged in a 19 by 19 or a 38 by 38 square respectively. The illuminated area of the DMD is independent of the number of pixels used in the superpixel.

The measured results are shown in Fig. 7.5. We see that at a large number of runs $N_{\text {run }}$ an increase in the number of controlled segments results in a higher enhancement. When $N_{\text {run }}$ is smaller than $10^{3}$ the enhancement is higher for a lower number of segments. When $N_{\text {run }}$ is greater than $10^{3}$ the enhancement is higher for a higher number of segments. Hence we conclude that for the higher number of the segments it takes longer to optimize a wavefront, but the optimized flux is higher than for optimization with the small number of segments. For the measurements performed in this report the total number of segments $N$ is 


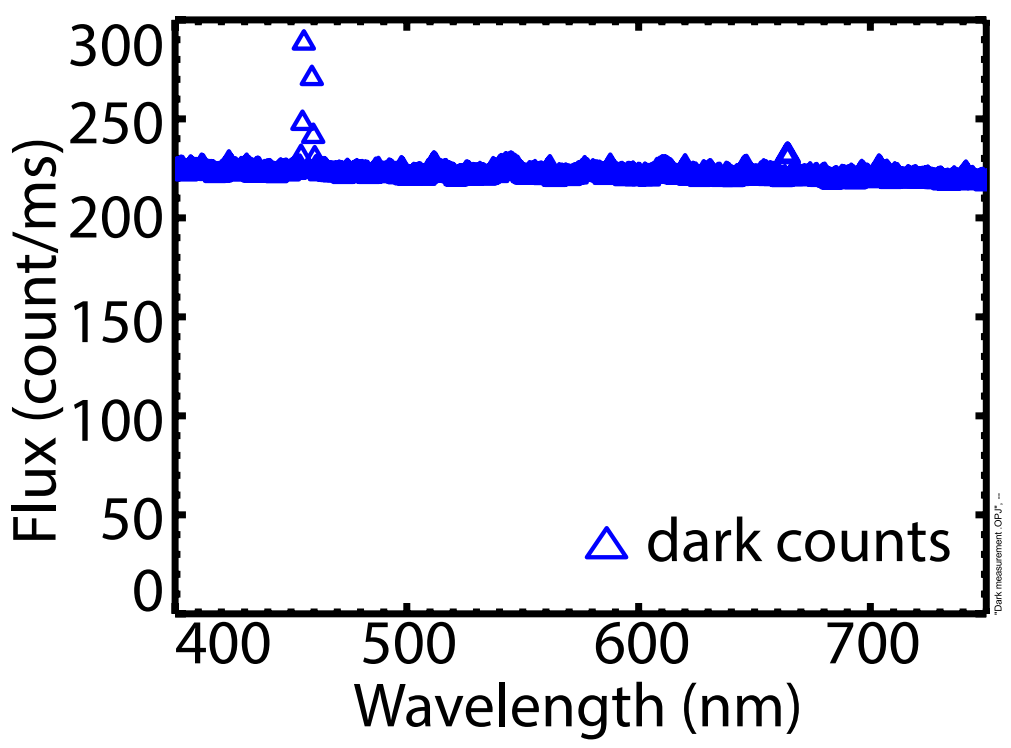

Figure 7.6: Dark counts measurement for the experimental setup. The standard deviation of the data points is within the symbol size. The prominent peak at $\lambda=457 \mathrm{~nm}$ is arising from stray light in the setup.

chosen to be 900 (30 by 30 segments) and the number of runs is selected to be $N_{\text {run }}=2000$. We use segments of the sizes of 4 by 4 superpixels.

\subsubsection{Detection system}

In this section, we discuss the characterization of the detection system notably the dark counts and the sensitivity. To correct for the dark counts of the spectrometer, we measure the signal with an integration time $T_{\mathrm{int}}=500 \mathrm{~ms}$ when the incident beam is blocked. The spectrum is averaged over 198 measurements and shown in Fig. 7.6. A peak at $\lambda=457 \mathrm{~nm}$ is observed, which coincides perfectly with the wavelength of the laser. The peak is caused by the stray light of the laser in the setup. Since this stray light is always present during the measurements, we take it into account. In this way, the stray light intensity is always subtracted from the real signal.

To calibrate the wavelength dependent sensitivity of the detection system, we measure a white LED that was characterized by Philips Lighting. Philips Lighting kindly provided a look-up table with light flux in watts for each wavelength emitted by white LED. The white LED light source is placed at the position of the sample, and the spectrum of the LED is recorded (see Fig. 7.7(a)). Standard spectrum $F_{\mathrm{c}}$ measured by Philips Lighting is compared to the signal $F_{\mathrm{m}}$ measured with our setup, so we can derive the calibration curve 

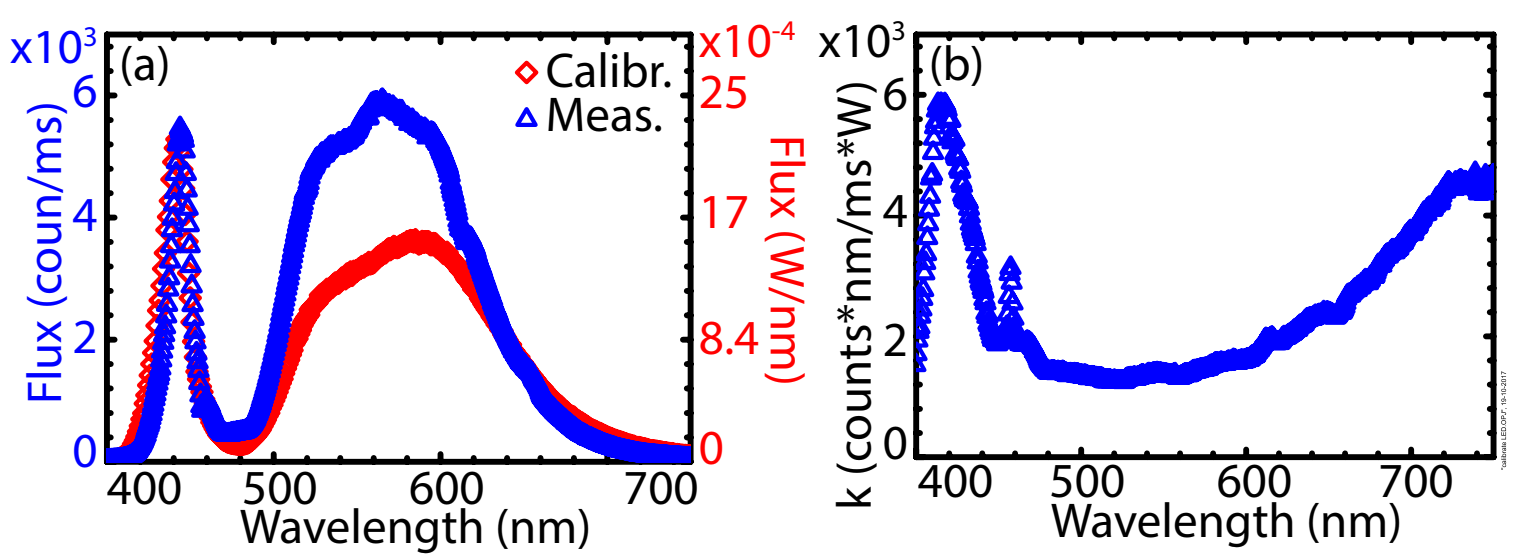

Figure 7.7: Calibration of the measurement setup. (a) The red spectrum is measured using calibrated equipment at Philips Lighting. The blue spectrum is measured in our setup. The ratio of these two spectra provides a calibration curve for our setup. (b) Calibration curve that is used to account for the wavelength dependence of the measurement setup.

$k(\lambda)$ of our setup using

$$
k(\lambda) \equiv \frac{F_{\mathrm{m}}(\lambda)}{F_{\mathrm{c}}(\lambda)},
$$

The calibration curve is plotted in Fig. 7.7(b). We see a highly nonlinear normalization curve with peaks between 400 and $500 \mathrm{~nm}$ and a steady increase of $k(\lambda)$ with the wavelength that starts around $500 \mathrm{~nm}$. The shape of this curve depends on the transmission characteristics of each component in the setup.

\subsubsection{Measurement procedure}

The blue laser illuminates the phosphor sample. The transmitted light that contains scattered and re-emitted light is registered by the spectrometer and CCD as described in Sec.7.3. The CCD registers the total flux emitted by the sample and cannot discriminate signal at multiple wavelengths. To discriminate the scattered and the re-emitted light on the CCD during the optimization run, we use long pass filter or short pass filter. The measurement procedure consists of the following steps: first, we place a long pass or short pass filter in front of the CCD to filter the scattered or the re-emitted part of the spectrum from the measured signal. The choice of the filter depends on the light signal that we want to optimize. To optimize on the scattered light we use a short pass filter with the cutoff wavelength of $\lambda=500 \mathrm{~nm}$, and to optimize on the re-emitted light we take a long pass filter with the cutoff wavelength of $\lambda=500 \mathrm{~nm}$. When the choice of the filter is made, we optimize the measured flux on CCD. The input parameters of the program are set to $N_{\text {run }}=2000, N=1444$ and the segment size is 4 by 4 superpixels. We record the spectrum 
Scattered light opt.
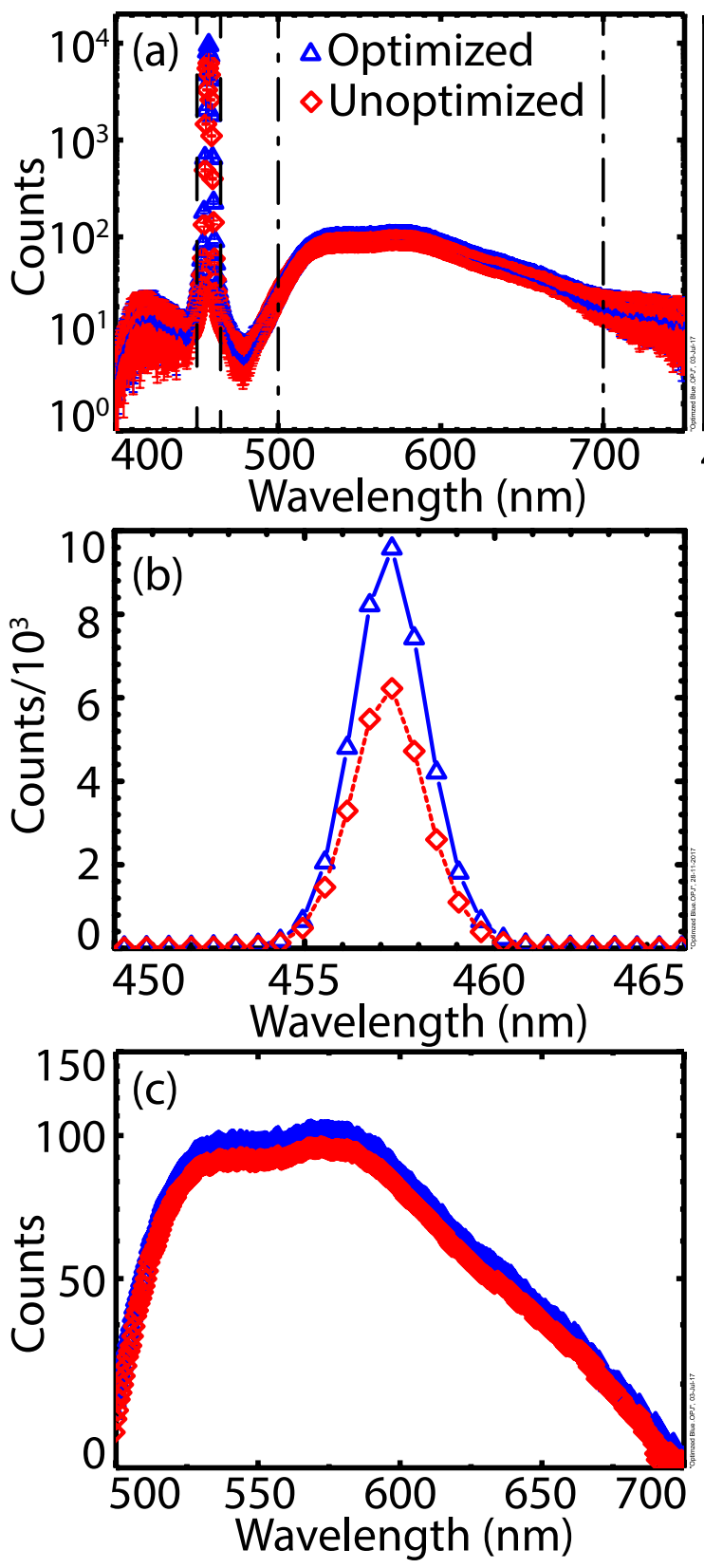

Re-emitted light opt.
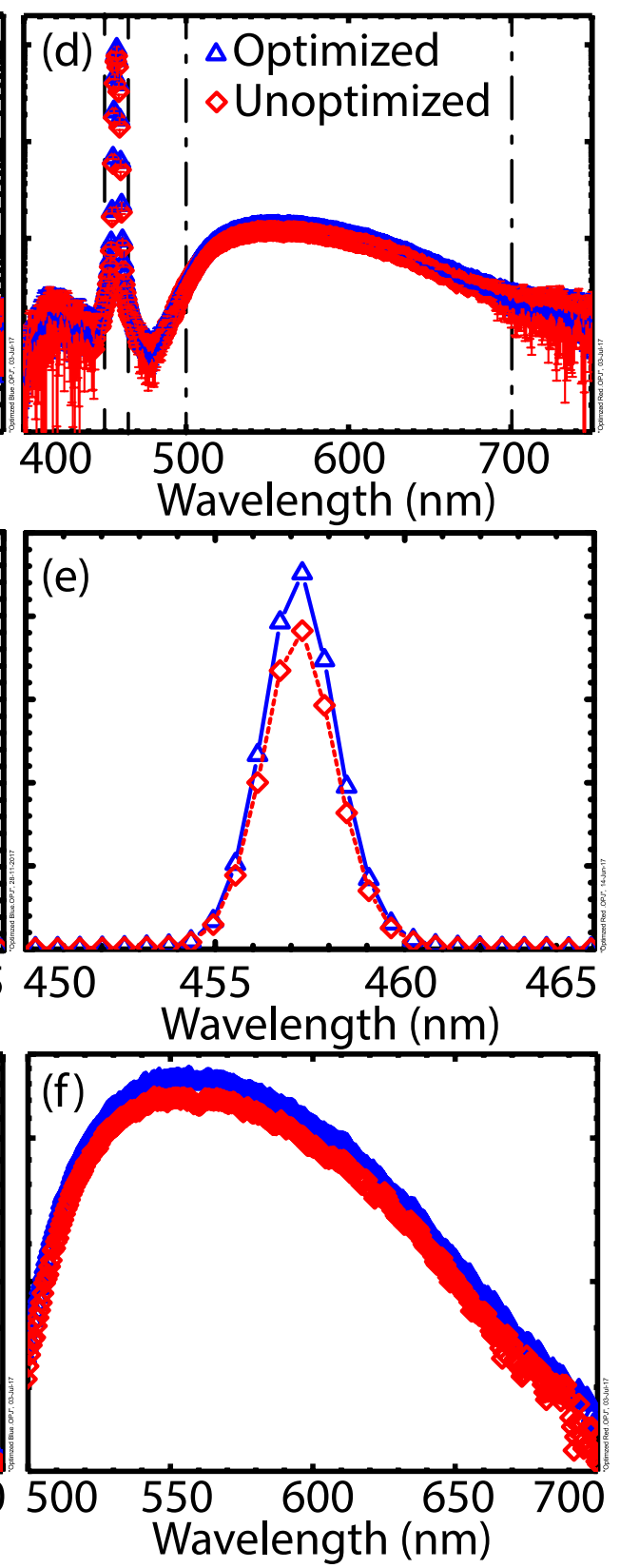

Figure 7.8: Optimized spectra of the model white LED. (a) Unoptimized and optimized spectrum measured when the optimization is performed on the scattered light. Dashed lines indicate the scattered light range, and dash-dotted lines indicate the re-emitted light. (b) Zoomed-in wavelength range of Fig. 7.8(a) of the scattered light. (c) Zoomed-in range of Fig. 7.8(a) of the re-emitted light. (d) Unoptimized and optimized spectrum measured when the optimization is performed on the re-emitted light. Dashed lines indicate the scattered light range, and dash-dotted lines indicate the re-emitted light. (e) Zoomed-in wavelength range of Fig. 7.8(d) of the scattered light . (f) Zoomed in range of Fig. 7.8(d) of the re-emitted light. 
of the optimized wavefront and random wavefront shown in Fig. 7.9(a-b) and discussed in the next section. The integration time of the spectrometer is set to $T=500 \mathrm{~ms}$. To calculate the standard deviation, we average over 99 measured spectra. The experiment is repeated 10 times for the scattered and for the re-emitted light to ensure reproducibility of the measured enhancement values. The achieved enhancement values are discussed in the next section.

\subsection{Results and discussion}

We illuminate the sample with the light source at the wavelength $\lambda=457 \mathrm{~nm}$. The incident light is being scattered, absorbed and re-emitted inside the sample. The transmitted, scattered and re-emitted light is measured using an integrating sphere and CCD. Typical measured signals are shown in Fig. 7.8(a) and (d). The scattered transmitted light flux has a pronounced peak at $\lambda=457 \mathrm{~nm}$ that coincides with the wavelength of the light source, as expected. The re-emitted light flux is spread between $\lambda=490 \mathrm{~nm}$ and $\lambda=700 \mathrm{~nm}$ and coincides with the re-emission band of the phosphor.

We use a wavefront shaping algorithm to optimize the light flux of the scattered light. The spectrum that is optimized on the scattered light spectrum and plotted in Fig. 7.8.(a). We see that the unoptimized spectrum has a lower flux at every wavelength in the visible range. To highlight the differences of the increased flux for the scattered and the re-emitted signal, we show the zoomed-in ranges of the spectrum in Fig. 7.8(b-c). In Fig. 7.8(b), we see the enhancement of the scattered light signal in the optimized case compared to the unoptimized case. The enhancement of the scattered light after optimization is $\eta_{\mathrm{s}}=$ $1.48 \pm 0.03$. The similar enhancement of the re-emitted light is apparent in Fig. 7.8(c). The enhancement of the re-emitted light is $\eta_{r}=1.074 \pm 0.007$. The enhancement of the re-emitted light is smaller than the enhancement of the scattered light. This difference in enhancements causes a shift of the color point of the transmitted light. We calculated the color point of the transmitted random and optimized light (see Fig. 7.1). The color point of the random wavefront is $x=0.244, y=0.191$, and for the optimized wavefront it is $x=0.223, y=0.155$. The error in the $x$ and $y$ coordinates is in the order of $1 \%$. To quantify the color shift caused by wavefront shaping, we define the relative color shift $\Delta_{\mathrm{d}}$

$$
\Delta_{\mathrm{d}} \equiv \sqrt{\Delta x^{2}+\Delta y^{2}}
$$

The color shift in our experiment is equal to $\Delta_{\mathrm{d}}=0.042 \pm 0.002$. For comparison, we 
calculate the color shift that occurs when the mass concentration of the phosphor particles is changed from $4 \mathrm{wt} \%$ to $5 \mathrm{wt} \%$. This color shift $\Delta_{\mathrm{d}}=0.03$ is taken as a reference, and we assign it to be $100 \%$. Wavefront shaped color shift advances the concentration color shift by $136 \pm 7 \%$. Hence, wavefront shaping technique allows to dynamically tune the color point without changing the phosphor concentration.

Next, we optimize the re-emitted light flux. The spectrum of the random and optimized light intensities is shown in Fig. 7.8(d). In Fig. 7.8(e-f) the enlarged parts of the spectra are shown for the scattered and the re-emitted light. Here we also observe an increase in the scattered and the re-emitted flux. The enhancement of the scattered light is $\eta_{\mathrm{s}}=1.26 \pm 0.09$, and the enhancement of the re-emitted light is $\eta_{\mathrm{r}}=1.10 \pm 0.02$. Although the optimization is done on the re-emitted light, the enhancement of the scattered light is higher than the enhancement of the re-emitted light. A possible reason might be that during the experiment more re-emitted flux is lost compared to the scattered flux. The re-emitted light has a uniform angular distribution, whereas the scattered light is more directional [97]. Hence, the collection objective with $\mathrm{NA}=0.65$ will collect predominantly forward scattered light and will miss light scattered at higher angles. Another possible reason is that the lower signal to noise ratio of the re-emitted light prevents the optimization process. During the experiment, we observed higher fluctuations of the re-emitted light flux compare to the scattered light flux. These fluctuations cause the optimization algorithm to perform worse [116]. We observed a difference in the shape of the re-emitted spectrum for the re-emitted light optimization and scattered light optimization (see Fig. 7.8(c) and (f)), however, the shapes of the spectra between optimized and unoptimized runs appear to be the same. The reason for such differences is currently unknown.

We calculated the color points of the unoptimized wavefront as $x=0.246, y=0.194$ and optimized wavefronts as $x=0.241, y=0.186$. The color point shift between optimized wavefronts and unoptimized wavefronts is $\Delta_{\mathrm{d}}=0.001 \pm 0.0005$, this corresponds to $3 \pm 2 \%$ of the shift compared to the reference shift defined above. In Table 7.1 we summarize the obtained results. It is apparent from Table 7.1 that the color point shift for the re-emitted light is one order of magnitude smaller than for the scattered light.

We plot the enhancements $\eta_{\mathrm{s}}$ and $\eta_{\mathrm{r}}$ as a function of the diffused spot area for the scattered and re-emitted light (see Fig. 7.9. We used Ref. 117, 118 for the theoretical predictions of the number of modes. The theoretical curves decay very rapidly with the area of the diffused beam for both absorbed and the re-emitted light. For the re-emitted light even when we consider a typical $\mu \mathrm{m}^{2}$ area, the theoretical enhancement is almost equal to 1 for our experimental setup and sample. Strikingly the experimental enhancement is 

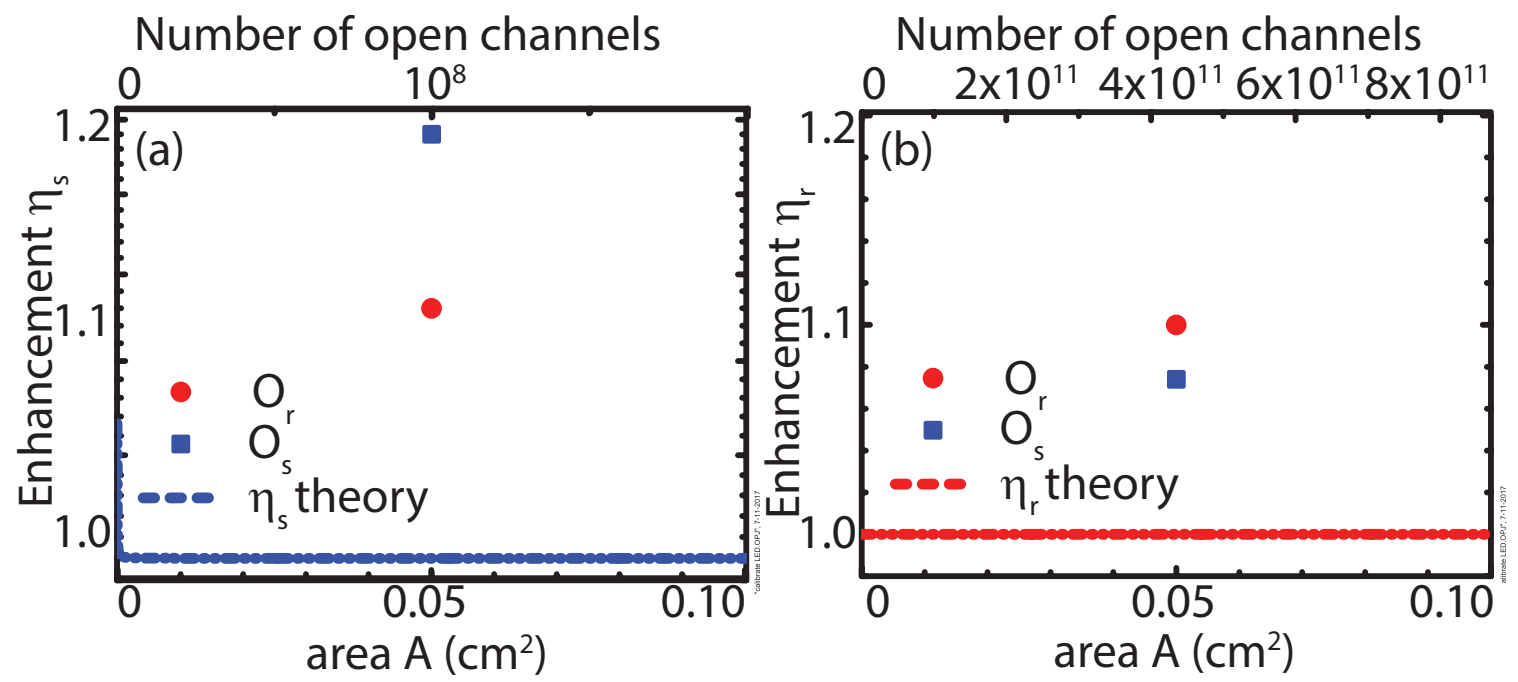

Figure 7.9: Enhancement as a function of target area for scattered and re-emitted light. (a) Enhancement as a function of the target area for the scattered light, where $O_{\mathrm{s}}$ is the enhancement of the scattered light and $O_{\mathrm{r}}$ is the enhancement of the re-emitted light. The data point is the measured value in this chapter. The top $x$ axis indicates the number of open channels in the system. (b) Enhancement as a function of the target area for the re-emitted light. Theoretical curves were generated following the predictions of Ref. 117, 118

well above the theoretically predicted values. It implies that even with the few control channels and with a macroscopically large number of open channels, the transmitted flux can be increased. Because the increase of flux for the scattered and re-emitted light is not equal, we observe the color point shift of the emitted light.

Table 7.1: Overview of measurement results

\begin{tabular}{|c|c|c|}
\hline & Optimized on scattered & Optimized on re-emitted \\
\hline$\eta_{s}$ & $1.48 \pm 0.03$ & $1.26 \pm 0.09$ \\
\hline$\eta_{r}$ & $1.074 \pm 0.007$ & $1.10 \pm 0.02$ \\
\hline$\Delta_{\mathrm{d}}$ & $0.042 \pm 0.002$ & $0.001 \pm 0.0005$ \\
\hline$\%$ shift & $136 \pm 7 \%$ & $3 \pm 2 \%$ \\
\hline
\end{tabular}

\subsection{Summary and outlook}

We have demonstrated the tuning of the color point of a white LED using wavefront shaping technique. When optimizing on the scattered light we obtain enhancements $\eta_{s}=1.48 \pm 0.03$ and $\eta_{r}=1.074 \pm 0.007$. This results in a color point shift of $\Delta_{\mathrm{d}}=0.042$ that corresponds 
to $36 \%$ of the color shift due to irreversible concentration change. For optimization on the re-emitted light we find $\eta_{s}=1.26 \pm 0.09$ and $\eta_{r}=1.10 \pm 0.02$, resulting in a color point shift of $\Delta_{\mathrm{d}}=0.001$, which corresponds to $3 \%$ of the shift due to concentration change. We observed that the color point always shifts to the blue part of the spectrum which is caused by the fact that the enhancement of the scattered light is always bigger than the enhancement of the re-emitted light.

We demonstrated that the wavefront shaping could be achieved even on macroscopic samples $\left(A \approx 5 \mathrm{~mm}^{2}\right)$. In this chapter, we showed for the first time that enhancement on macroscopic objects in the re-emitted light could be achieved. The existing theories cannot describe the obtained enhancements. Ojambati et al. reported enhancements $\eta=2$ on areas of $A=15 \mu \mathrm{m}^{2}$, and it is surprising to see that an increase in the optimization area by 3 orders of magnitude resulted for our strongly absorbing samples in a comparable enhancement 115]. The enhancement of the transmitted flux demonstrated in this chapter paves the way to a white LED efficiency enhancement based on passive optical elements, such as the phase masks.

In conclusion in this chapter, we demonstrated the proof of principle reversible color tuning of a white LED. We have been able to wavefront shape light in a macroscopic area on strongly absorbing scattering samples with re-emission. We observed that both scattered and re-emitted light is enhanced in the process of wavefront shaping. The reached enhancements are comparable to the enhancements achieved in non-absorbing samples for $\mu \mathrm{m}^{2}$ area. 


\section{Chapter 8}

\section{Summary}

Lighting is a crucial technology that is used every day. The introduction of the white light emitting diode (LED) that consists of the Nobel Prize-winning technology of a blue LED combined with a phosphor layer, dramatically reduces the energy consumption for lighting. Despite the fast-growing market penetration, there are currently only trial and error approaches for white LED modeling. In this thesis, I define experimental and theoretical tools that analytically connect phosphor powder properties to the color point of a white LED. Phosphor particles inside the diffuser layer scatter, absorb and re-emit incident light. Absorption and scattering comparably affect light; hence, neither of the effects can be neglected. Due to these properties, widely used analytical tools fail to describe phosphor diffusion layers. I propose and experimentally verify a novel systematic approach that fully describes light diffusion and re-emission in phosphor layers.

The P3 approximation was chosen to be the central theoretical framework that allowed us to describe light propagation in scattering materials with strong absorption. This approximation is derived from the more general radiative transfer equation (RTE), and detailed steps of derivations are described in Chapter 2. The equations of the P3 approximation in the parallel slab geometry are analytically solvable, and presented at the end of the Chapter 2 .

The $\mathrm{P} 1$ approximation is the first order approximation to RTE, and it has a much simpler analytical solution than the P3 approximation. In Chapter 6, it was shown that the P1 approximation gives a substantial error of the order of $30 \%$ in estimating transport parameters for the scattering materials with strong absorption, and it cannot be applied to some scattering materials. To identify the range of validity for the P1 approximation, I derive analytical expressions and 2D maps that provide error estimates of the P1 approximation for the whole transport parameter range.

To analyze data with the P1 and the P3 approximations, I performed measurements 
in the range where scattered and re-emitted light are spectrally separated. Conventional measurement techniques fail to provide such separation when scattering materials with broadband re-emission are studied. In Chapters 3 and 4 I present a novel experimental setup that solves the problem. This setup uses narrowband illumination, so the scattered and re-emitted light is always spectrally separated or can be distinguished.

The central objective of Chapters 3 and 4 was to identify the transport parameters of the phosphor plates that are typically used for white LEDs. In Chapter 3, the extracted parameters were $\left(\ell_{\mathrm{tr}}, \ell_{\mathrm{a}}\right)$, and the $\mathrm{P} 1$ approximation was used to extract these parameters. In Chapter 44 a more sophisticated experiment was performed, and it allowed to identify a wider parameter space $\left(\ell_{\mathrm{tr}}, \ell_{\mathrm{a}}, \mu\right)$. In this case, the P3 approximation was used to analyze experimental data.

Once transport parameters were identified, I used the P3 approximation for scattered and re-emitted light to analytically predict the spectrum of a white LED. This approach is described in Chapter 5. The spectrum of light can be easily converted to the color point. The conversion process is described in Appendix A. In this chapter, the analytical connection between the transport parameters of the phosphor and the color point of a light source was presented. This approach opens vast possibilities for the systematic design of white LEDs. A look-up table can be created where the phosphor type will be mapped to the area of colors it can generate. Application of the described approach will provide the design of the thickness of the phosphor layer $d$ and the phosphor concentration $\rho$ that will produce the desired color.

In Chapter 5 it was shown that design parameters strictly define the color point of the light source. I demonstrated in Chapter 7 that the color point of a white LED can be reversibly tuned without changing its design. This tuning is possible when the wavefront shaping technique is used to enhance the intensity at a preset wavelength. Theoretical predictions show that this technique works efficiently only for areas of illumination that are $\mu \mathrm{m}$ in size. In Chapter 7, it was demonstrated that enhancement reaches almost 1.5 for $\mathrm{mm}$ size areas of illumination. The enhancement of light at different wavelengths had different magnitude, which allowed reversible tuning of the color point.

I have developed tools that can drastically change the white LED design process, allowing analytical methods to be widely used. It will enable access to a large design parameter space by using an analytical algorithm without adjustable parameters. While my current solutions provide a one-dimensional description of the light propagation, the analytical solutions of the P3 approximation in the 3D geometry is already available. It describes the light propagation in complex geometries that are regularly employed in white LED design. My 
approach increases the design efficiency by avoiding recurring design efforts and decrease the cost of ownership of white LEDs units for worldwide users. 


\section{Nederlandse samenvatting}

Verlichting is een cruciale technologie die elke dag wordt gebruikt. De introductie van de witte lichtgevende diode (LED) dit is gebaseerd op de Nobelprijs-winnende technologie van een blauwe LED in combinatie met een fosforlaag, heeft het energieverbruik voor verlichting drastisch verminderd. Ondanks de sneltoenemende marktpenetratie, zijn er momenteel slechts een klein aantal LED ontwerpmethodes. In dit proefschrift beschrijf ik door mij ontwikkelde experimentele en theoretische hulpmiddelen die de eigenschappen van fosforpoeder analytisch verbinden met het kleurpunt van een witte LED. Fosfordeeltjes in de diffusorlaag verstrooien, absorberen en geven invallend licht opnieuw af. Absorptie en verstrooiing hebben een vergelijkbare invloed op het licht uitgezonden door een LED. Daarom kunnen geen van beide effecten worden verwaarloosd. Vanwege hoge absorptie kunnen veelgebruikte analytische hulpmiddelen de optische eigenschappen van fosfordiffusielagen niet beschrijven. In dit proefschrift stel ik experimentele en theoretische hulpmiddelen voor die met een nieuwe systematische benadering van lichtdiffusie en re-emissie in fosforlagen volledig beschrijven.

De P3-benadering is gekozen als het centrale theoretische raamwerk dat ons in staat stelt de lichtvoortplanting in verstrooiende materialen met een sterke absorptie te beschrijven. Deze benadering is afgeleid van de meer algemene stralingsoverdrachtsvergelijking (radiative transfer equation - RTE); gedetailleerde stappen van afleidingen worden beschreven in hoofdstuk 2. De vergelijkingen van de P3-benadering voor een parallelle plaat zijn analytisch oplosbaar en gepresenteerd aan het einde van hoofdstuk 2.

De P1-benadering is de eerste-orde benadering van RTE en heeft een veel eenvoudigere analytische oplossing dan de P3-benadering. In hoofdstuk 6 is aangetoond dat de P1-benadering een aanzienlijke fout geeft, van de orde van $30 \%$, bij het schatten van transportparameters voor de verstrooiende materialen met sterke absorptie, en op sommige materialen zelf niet kan worden toegepast. Om het geldigheidsbereik voor P1-benadering te identificeren, leid ik analytische expressies af die foutschattingen van de P1-benadering voor het hele bereik van de transportparameters bieden. Deze schattingen worden gepresenteerd 
in de vorm van 2D-kaarten.

Om gegevens te analyseren met de P1- en de P3-benaderingen, heb ik metingen uitgevoerd in het bereik waar verstrooid licht en opnieuw uitgestraald licht spectraal gescheiden worden. Conventionele meettechnieken leveren een dergelijke scheiding bij de bestudering van verstrooiende materialen met breedbandemissie niet op. In de hoofdstukken 3 en 4 presenteer ik een nieuwe experimentele opstelling die het probleem oplost. Deze opstelling maakt gebruik van smalband-verlichting, waardoor het verstoorde en opnieuw uitgestraalde licht altijd kan worden onderscheiden.

Het centrale doel van de hoofdstukken 3 en 4 is om de transportparameters van de fosforplaten te identificeren die typisch worden gebruikt voor witte LED's. In hoofdstuk 3 zijn de parameters $\left(\ell_{\mathrm{tr}}, \ell_{\mathrm{a}}\right)$, en de P1-benadering gebruikt om deze parameters te extraheren. In Hoofdstuk 4 is een meer verfijnd experiment uitgevoerd en kon een bredere parameterruimte $\left(\ell_{\mathrm{tr}}, \ell_{\mathrm{a}}, \mu\right)$ worden geïdentificeerd. In dit geval is de P3-benadering gebruikt om experimentele gegevens te analyseren.

Nadat de transportparameters zijn geïdentificeerd, gebruik ik de P3-benadering voor verstrooid en opnieuw uitgezonden licht om het spectrum van een witte LED analytisch te voorspellen. Deze aanpak is beschreven in hoofdstuk 5. Het spectrum van het licht kan eenvoudig worden omgezet in het kleurpunt. Dit conversieproces wordt beschreven in Bijlage A. In dit hoofdstuk wordt de analytische verbinding tussen de transportparameters van de fosfor en het kleurpunt van een lichtbron gepresenteerd. Deze aanpak opent enorme mogelijkheden voor het systematisch ontwerpen van witte LED's. Er kan een opzoektabel worden gemaakt waarin het fosfortype wordt toegewezen aan het gebied met kleuren dat het kan genereren. Toepassing van de beschreven aanpak zal de dikte van de fosforlaag $d$ en de fosforconcentratie $\rho$ voorschrijven die de gewenste kleur zal produceren.

In hoofdstuk 5 wordt aangetoond dat de ontwerpparameters het kleurpunt van de lichtbron uniek bepalen. In hoofdstuk 7 heb ik aangetoond dat het kleurpunt van de witte LED omkeerbaar kan worden gevarieerd zonder het ontwerp te veranderen. Deze afstemming is mogelijk wanneer een golffrontaanpaszingtechniek wordt gebruikt om de intensiteit bij een vooraf ingestelde golflengte te verbeteren. Theoretische voorspellingen hadden aangetoond dat golffrontaanpassing efficiënt werkt voor verlichtgebieden die $\mu \mathrm{m}$ groot zijn. In hoofdstuk 7 word echter aangetoond dat golffrontaanpassing leidt tot een verbetering met een factor van bijna 1.5 bij verlichtingsgebieden met een mm-grootte. De toename van het licht verschilt voor verschillende golflengtes, wat omkeerbare afstemming van het kleurpunt mogelijk maakte.

Ik heb theoretischemiddelen ontwikkeld die het witte LED-ontwerpproces drastisch 
kunnen veranderen, waardoor analytische methoden op grote schaal kunnen worden gebruikt. Het zal een grote ontwerpparameterruimte mogelijk maken met behulp van een analytisch algoritme zonder aanpasbare parameters. Hoewel mijn huidige oplossingen een ééndimensionale beschrijving van de lichtvoortplanting bieden, zijn de analytische oplossingen van P3-benadering in 3D-geometrieën al beschikbaar. Ze kunnen lichtvoortplanting in complexe geometrieën beschrijven die regelmatig in witte LED-ontwerpen worden gebruikt. Mijn aanpak verhoogt de ontwerpefficiëntie door terugkerende ontwerpinspanningen te vermijden en de eigendomskosten van witte LED's voor wereldwijde gebruikers te verlagen. 


\section{Appendix A}

\section{Color point}

\section{A.1 What is color?}

Color is a complicated concept that includes both a strict physical definition and human psychology - a rare combination. To demonstrate the psychological aspect of this problem, we will discuss the famous case of 'The dress.' On 26 February 2015 on the social service Tumblr a user posted a washed-out color photograph of a dress with hashtag \#thedress, shown in Fig. A.1. Users were asked to answer a simple question: 'what is the color of the dress?' It appears that only $57 \%$ of the people perceived the dress as blue and black. An other $30 \%$ saw it as gold and white, and $2 \%$ saw it as something else 120].

The scientist suggested that the perception of colors on the photo depends on the white light source used to illuminate the object and how the brain processes this illumination [121. The final answer to this question is a topic of ongoing scientific investigation in neuroscience and vision science. This example illustrates the psychological aspects of color perception and importance of a white light source characteristics in our lives.

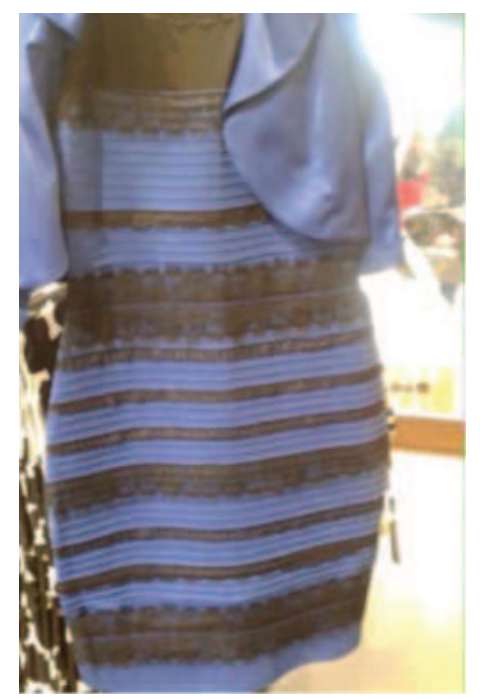

Figure A.1: 'The dress.' The color of the dress is blue and black. It may appear white and golden. Figure adopted from [120]. Dress image reproduced with permission from Cecilia Bleasdale. Although color perception depends on the individual, there were formulated various methods to characterize color of objects, light sources, and their interaction [93]. These methods include rigorous characterization of the eye photodetector sensitivity. In this appendix, we describe how to identify the color perceived by a human 


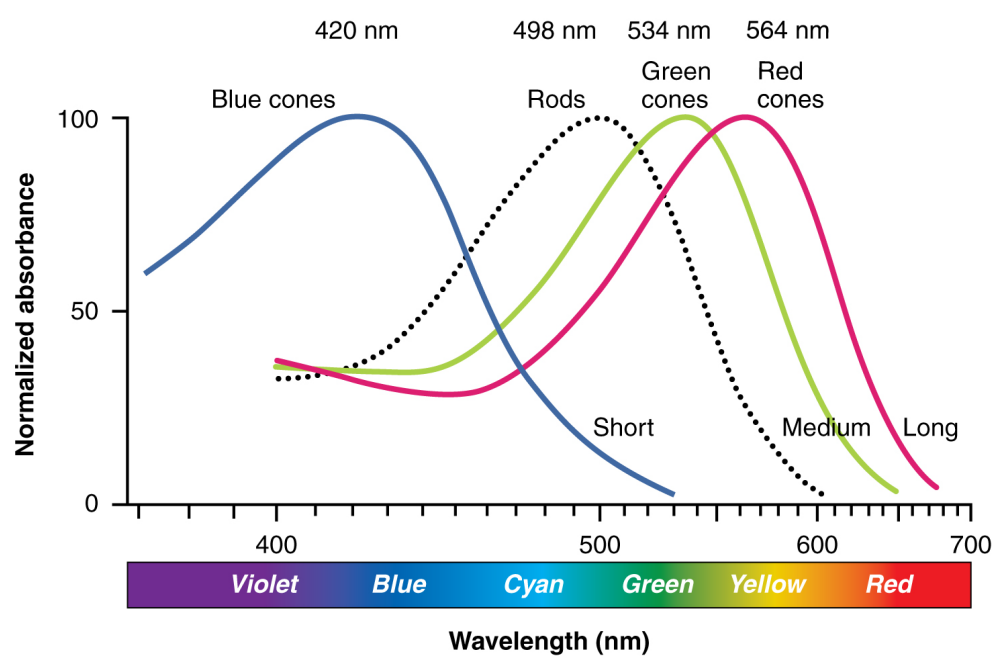

Figure A.2: Absorbance curve of the eye sensors. The sensitivity of rods and cones of the eye as a function of wavelength. Figure adopted from [122].

from the spectrum measured in the lab.

\section{A.2 Human eye}

The human eye contains two types of photodetectors: rods and cones [55]. Rods are the color insensitive type of photodetectors. They are optimized for night vision and hence sensitive to deficient light levels. The absorbance curve as a function of wavelength for the rods is shown in Fig. A.2. Cones are color sensitive photodetectors. There are three types of cones red, green and blue (see Fig. A.2). Every type of cone is optimized to be sensitive in its own part of the spectrum. The absorbance curves for all three types of detectors overlap, covering all visible spectrum. Cones perform well at high light levels and are mainly used during the day. There are about 120 million rods and 6 million cones inside the human eye.

The distribution of cones and rods is not uniform over the surface of the eye (see Fig. A.3). The cones are mainly concentrated in the center of the eye, and rods are concentrated on the periphery. Cones have a higher spatial resolution compared to rods. There are no photoreceptors at an angle of 20 degrees from the fovea of the eye. That is the position where the eye nerve is connected to the eye, and this area is called the blind spot.

\section{A.3 From spectrum to color}

The visible spectrum is a range of electromagnetic radiation that can be registered by our eye 55]. Each wavelength in the visible spectrum corresponds to the spectrally pure 


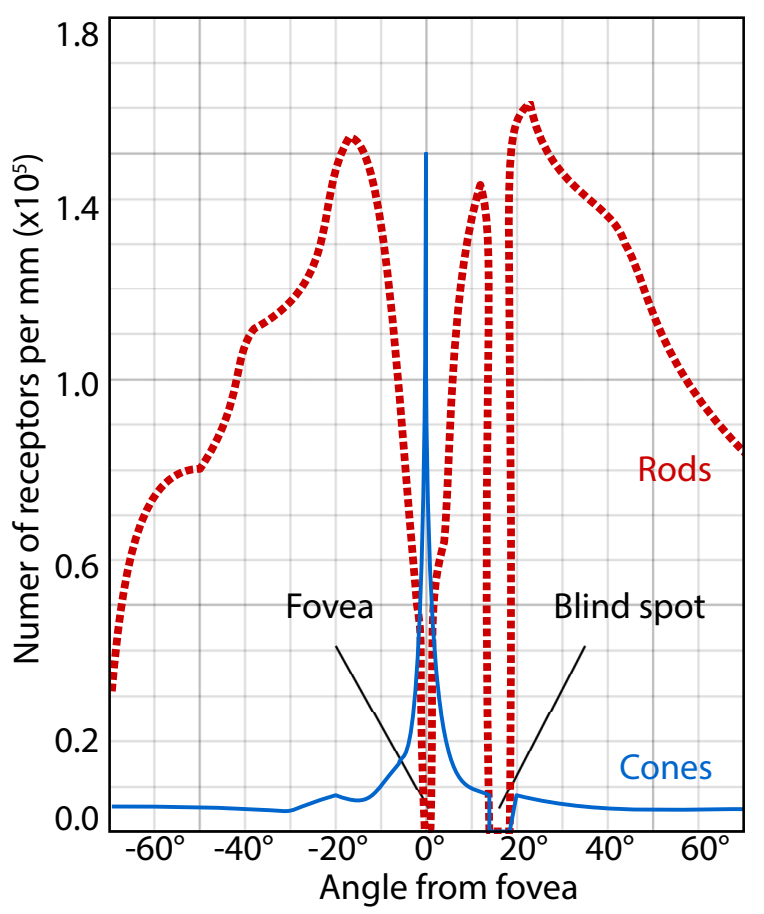

Figure A.3: The density of rods and cones in the eye. The distribution of cones and rods inside the eye as a function of the angle from the fovea. Figure adopted from [123].

monochromatic color seen by the human. The spectrally pure colors are not restricted to the variety of colors in nature. The purple color is a combination of blue and red spectrally pure colors. Orange is a spectrally pure color $(600 \mathrm{~nm})$, it can also be produced by mixing red $(700 \mathrm{~nm})$ and yellow $(580 \mathrm{~nm})$. The eye cannot detect the two spectral components that resemble orange color, so we say that the eye is a synthesizer. On the contrary, the ear is an analyzer, because when we listen to an orchestra, separate instruments can be heard.

Colors can be described using three parameters. Here we use hue, saturation, and luminance (see Fig. A.4). Saturation characterizes the amount of white color added to the spectrally pure color. For example, if we add white to red color, we will obtain the pink color. The saturation is $100 \%$ if no white is added. To define luminance, we consider the following examples. If two objects with the identical color are illuminated differently (one object is in the darkness, and another one is strongly illuminated), they appear to have a different color. This is the essence of the luminance parameter.

The CIE 1931 color space is the first quantitative method to convert color to spectrum and vice versa. One of the most widely known color space is the RGB color space, which stands for red, green and blue color space. To define the main quantities of this space the color matching experiments was done (see Fig. A.5). An observer looks at the screen that is divided into two parts as shown in Fig. A.5(a). One part of the screen is illuminated 


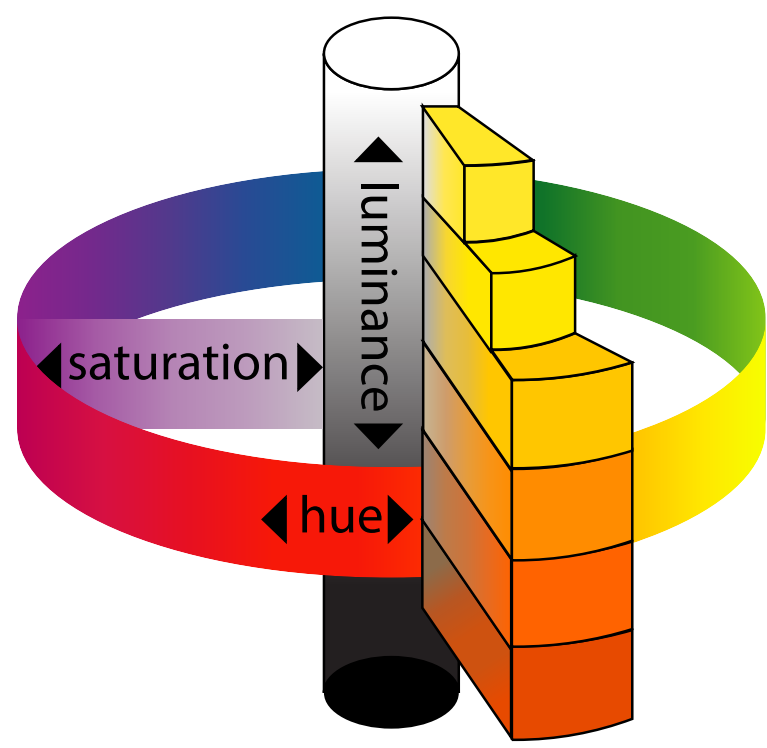

Figure A.4: Hue, saturation and luminance diagram. Hue changes on the circumference of the circle. Saturation changes along the diameter of the circle. Luminance changes on the axes that cross the center of the circle perpendicularly.

with three spectrally pure colors (red, green and blue). Another part is illuminated with arbitrary color. The observer is asked to match the color of the two screens by adjusting the power of the three light sources. When the colors have matched, the value of the individual power settings is recorded as a function of wavelength (see Fig. A.5). When the color of the screens cannot be matched exactly then the additional light source is used to illuminate the reference screen. The power of the additional source is recorded as negative power. In Fig. A.5(b) we show functions $\bar{x}, \bar{y}$ and $\bar{z}$, and they are already normalized in such a way that no negative values are present.

To obtain the RGB coordinates of the measured spectrum the following mathematical procedures should be performed. First we calculate the tristimulus values of the spectrum

$$
\begin{aligned}
& X=k \sum_{\mathrm{i}} \Phi\left(\lambda_{\mathrm{i}}\right) \bar{x}\left(\lambda_{\mathrm{i}}\right) \Delta \lambda_{\mathrm{i}}, \\
& Y=k \sum_{\mathrm{i}} \Phi\left(\lambda_{\mathrm{i}}\right) \bar{y}\left(\lambda_{\mathrm{i}}\right) \Delta \lambda_{\mathrm{i}}, \\
& Z=k \sum_{\mathrm{i}} \Phi\left(\lambda_{\mathrm{i}}\right) \bar{z}\left(\lambda_{\mathrm{i}}\right) \Delta \lambda_{\mathrm{i}},
\end{aligned}
$$

where $k$ is the normalization constant that often chosen in such way that $Y=100$ for the white color. The procedure of calculating the tristimulus value is shown in Fig. A.6, $X Y Z$ can be easily converted to $R G B$ using a conversion matrix. The conversion matrix depends on the choice of the chosen white color spectrum. In this thesis we use white color D65 
(a)
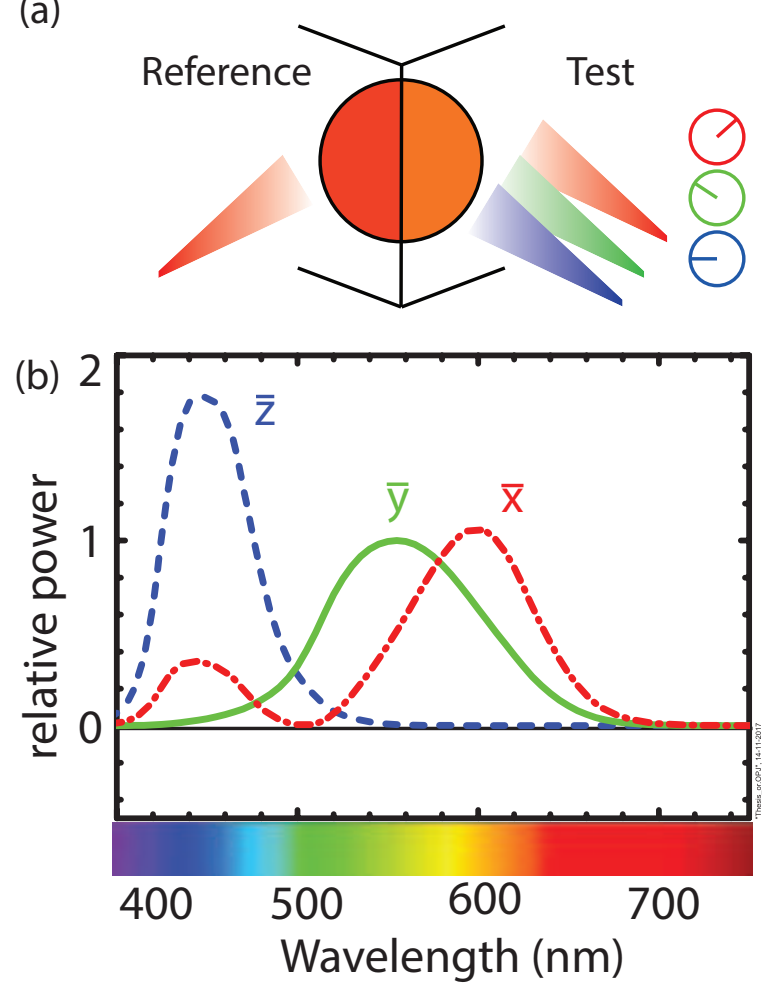

Figure A.5: Color matching experiment. (a) The color matching experimental setup. The color is set on the left part of the screen - the reference screen. Knobs on the right side of the screen are adjusted to match the color of the test screen to the reference screen. (b) The color matching functions $\bar{x}, \bar{y}$ and $\bar{z}$.
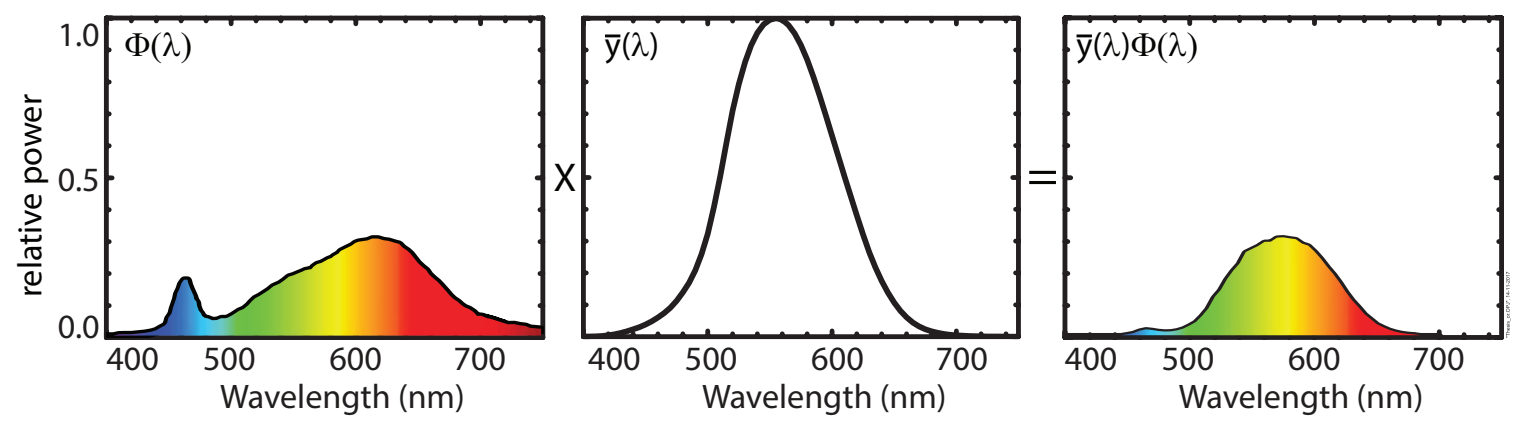

Figure A.6: From color space $\bar{y}$ to color space $Y$. The light source with the spectral density $\Phi\left(\lambda_{\mathrm{i}}\right)$ is multiplied by the color matching function of the eye $\bar{y}\left(\lambda_{\mathrm{i}}\right)$ to obtain the stimulus registered by the human eye $\bar{y}\left(\lambda_{\mathrm{i}}\right) \Phi\left(\lambda_{\mathrm{i}}\right)$. When we sum $\bar{y}\left(\lambda_{\mathrm{i}}\right) \Phi\left(\lambda_{\mathrm{i}}\right)$ over all $i$ 's in the visible spectrum we receive $Y$ that is the area under the curve in the last figure.

shown in Fig. A.7, D65 roughly represents the average midday light in Western Europe. It is a combination of the sunlight spectrum and light diffused by the atmosphere. We chose $Y=100$ to define $k=1.1149$, as mentioned above.

In this thesis we used chromaticity values to indicate the color point on the color space. 


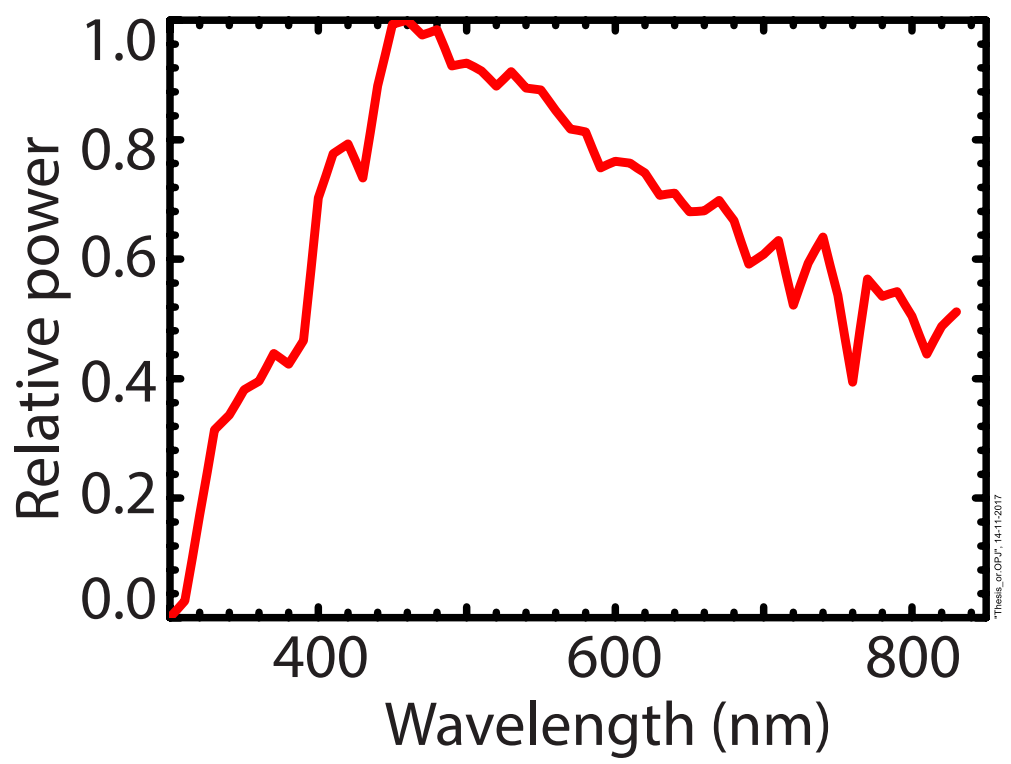

Figure A.7: Spectrum of the white color D65. The chromaticity values of the $D 65$ are $(x, y)=(0.3125,0.3290)$.

Chromaticity values $x y z$ are calculated as follows

$$
\begin{aligned}
& x=\frac{X}{X+Y+Z}, \\
& y=\frac{Y}{X+Y+Z}, \\
& z=1-x-y .
\end{aligned}
$$

These values are used as coordinates of the color point in the color space.

\section{A.4 Color of objects}

The color of an object depends on two factors: the reflectance of the object as a function of wavelength and the power spectrum of the light source that illuminates the object. In this section, we do not consider the psychological aspects described in Sec. A.1. In Fig. A.8 we show how the spectrum of the illumination influences the color perceived by the eye. The red object with reflectance spectrum shown in Fig. A.8(b) is illuminated by a RGB LED with the power spectrum shown in Fig. A.8(a). The stimulus at the eye is calculated by multiplying the power spectrum of the source by the reflectance spectrum of the object. The result of the multiplication is shown in Fig. A.8(c). We converted the spectra shown in Fig. A.8(b-c) to the color perceived by the human eye. The results of the conversion are shown in the inset of Fig. A.8(b). The color of the middle colored square indicated with 


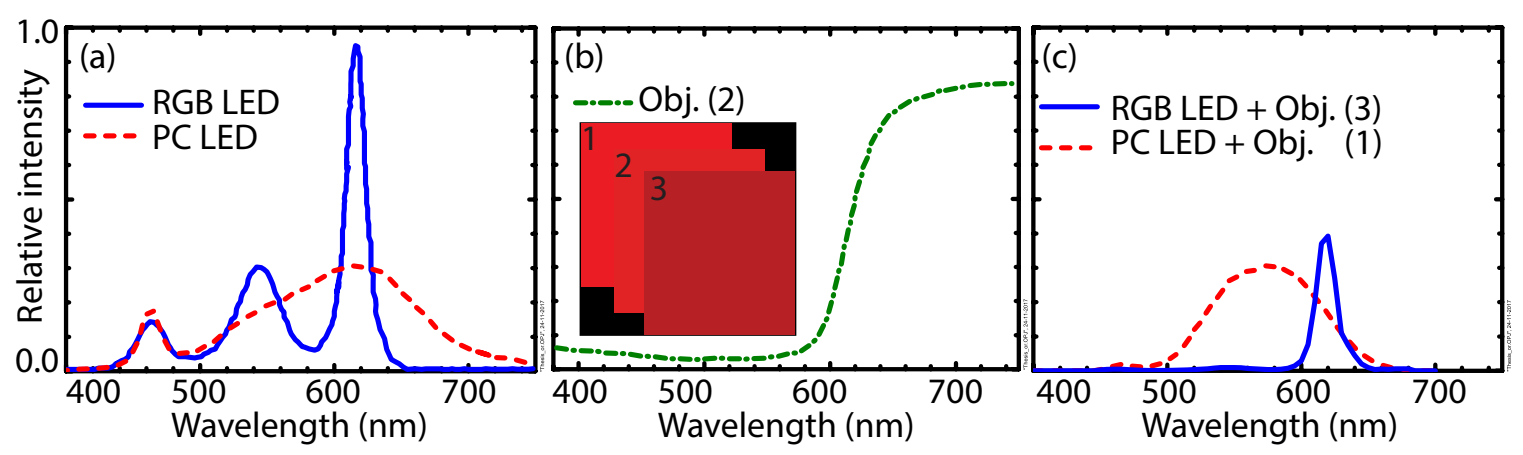

Figure A.8: Color vs illumination conditions. (a) Spectra of two white light sources that illuminate a test object. (b) The reflectance spectrum of the test object. The inset shows three squares with different shades of the red color. These colors are the result of the conversion of spectra from panels (b-c). Each color is indicated with the number that corresponds to the spectrum number mentioned in the legend of panels (b-c). (c) The stimulus registered by the eye.

number 2 represents the real color of the object. Squares 1 and 3 represent color perceived by the eye when illuminated with two different light sources. We see that the shape of the spectrum of the object is radically different from the spectra perceived by the eye. However, the resulting colors are very similar. Colors 1 and 2 can hardly be distinguished. 


\section{Bibliography}

[1] J. Goudsblom. Fire and Civilization. Penguin books, London, 2007.

[2] T.A. Edison. Electric lamp, 1880. US Patent 223,898.

[3] European Union statistics, See http://ec.europa.eu/eurostat/ statistics-explained/index.php/File:Share_of_fuels_in_the_final_ energy_consumption_in_the_residential_sector_by_type_of_end-use,_2015_ (\%25).png. Accessed 14 December 2017.

[4] T. Ezuhara, S. Miyazaki, and K. Toda. Phosphor for white led and a white led, 2003. US Patent App. 10/412,270.

[5] H. Amano, M. Kito, K. Hiramatsu, and I. Akasaki. P-type conduction in mg-doped gan treated with low-energy electron beam irradiation. Jpn. J. Appl. Phys., 28:L2112, 1989.

[6] S. Nakamura, N. Iwasa, M. Senoh, and T. Mukai. Hole compensation mechanism of p-type gan films. Nat. Photon., 3:180-182, 2009.

[7] N. Horiuchi. Light-emitting diodes: Natural white light. Nature, 4:738, 2010.

[8] E. F. Schubert. Light-Emitting Diodes. Cambridge University Press, London, second edition, 2006.

[9] Editorial. Haitz's law. Nat. Photon., 1:23, 2007. 
[10] T. Pulli, T. Donsberg, T. Poikonen, F. Manoocheri, P. Karha, and E. Ikonen. Advantages of white led lamps and new detector technology in photometry. Light Sci. Appl., 4:e332, 2015.

[11] Danish Energy Agency, Energy Piano CLASP, See http://clasp.ngo/ /media/ Files/SLDocuments/2015/DEA\%20-\%20CLASP\%20Report\%20on\%20European\%20LED\% 20Market_final.ashx, 2016. Access 24 October 2017.

[12] A. de Almeida, P. Fonseca, B. Schlomann, and N. Feilberg. Characterization of the household electricity consumption in the eu, potential energy savings and specific policy recommendations. Ener. Build., 43(8):1884 - 1894, 2011.

[13] S. Pimputkar, J. S. Speck, S. P. DenBaars, and S. Nakamura. Prospects for led lighting. Jpn. J. Appl. Phys., 31:1258, 1992.

[14] USA government. L prize, 2016.

[15] C. Gilray and I. Lewin. Monte carlo techniques for the design of illumination optics. Illuminating Engineering Society of North America Annual Conference Technical Papers (IESNA). Paper number 85, pages 65-80, 1996.

[16] C. Sommer, R. Krenn, J., P. Hartmann, P. Pachler, M. Schweighart, S. Tasch, and P. Wenzl, F. The effect of the phosphor particle sizes on the angular homogeneity of phosphor-converted high-power white led light sources. IEEE J. Quantum Electron., 2009.

[17] Z. Liu, S. Liu, K. Wang, and X. Luo. Measurement and numerical studies of optical properties of yag:ce phosphor for white light-emitting diode packaging. Appl. Opt., $49(2): 247-257,2010$. 
[18] T. W. Tukker. Fluorescence modeling in remote and close led illumination devices. SPIE International Optical Design Conference 2010 (SPIE,2010), Paper no. ITuE2, 2010.

[19] E. Hecht and A. Zajac. Optics. Addison Wesley, 1974.

[20] K. A. O’Donnell and E. R. Mendez. Experimental study of scattering from characterized random surfaces. J. Opt. Soc. Am. A, 4(7):1194-1205, 1987.

[21] E. G. van Putten, D. Akbulut, J. Bertolotti, W. L. Vos, A. Lagendijk, and A. P. Mosk. Scattering lens resolves sub-100 nm structures with visible light. Phys. Rev. Lett., 106:193905, 2011.

[22] H. Yilmaz, E. G. van Putten, J. Bertolotti, A. Lagendijk, W. L. Vos, and A. P. Mosk. Speckle correlation resolution enhancement of wide-field fluorescence imaging. Optica, $2(5): 424-429,2015$.

[23] A. Lagendijk and B. A. van Tiggelen. Resonant multiple scattering of light. Phys. Rep.,, 270(3):143 - 215, 1996.

[24] D. J. Durian. Influence of boundary reflection and refraction on diffusive photon transport. Phys. Rev. E, 50:857-866, 1994.

[25] M. C. W. van Rossum and T. M. Nieuwenhuizen. Multiple scattering of classical waves: microscopy, mesoscopy, and diffusion. Rev. Mod. Phys., 71:313-371, 1999.

[26] E. Akkermans and G. Montambaux. Mesoscopic Physics of Electrons and Photons. Cambridge University Press, 2007.

[27] A. P. Mosk, A. Lagendijk, G. Lerosey, and M. Fink. Controlling waves in space and time for imaging and focusing in complex media. Nature Photonics, 2012.

[28] S. Wiersma, D. Disordered photonics. Nat. Photon., 2013. 
[29] S. Rotter and S. Gigan. Light fields in complex media: Mesoscopic scattering meets wave control. Rev. Mod. Phys., 89:015005, 2017.

[30] M. R. Krames, O. B. Shchekin, R. Mueller-Mach, G. O. Mueller, Z. Ling, G. Harbers, and M. G. Craford. Status and future of high-power light-emitting diodes for solidstate lighting. J. Disp. Tech., 2007.

[31] H. Bechtel, P. Schmidt, W. Busselt, and S. Schreinemacher, B. Lumiramic: a new phosphor technology for high performance solid state light sources. Proc. SPIE, 7058:70580E-70580E-10, 2008.

[32] W. M. Star, J. P. A. Marijnissen, and M. J. C. van Gemert. Light dosimetry in optical phantoms and in tissues: I. multiple flux and transport theory. Phys. Med. Biol., 33(4):437, 1988.

[33] W. M. Star. Comparing the p3-approximation with diffusion theory and the with monte carlo calculations of light propogation in a slab geometry. In SPIE Instit. Ser. IS, volume 5, page 146. American Physical Society, 1989.

[34] J. W. Pickering, S. A. Prahl, N. van Wieringen, J. F. Beek, H. J. C. M. Sterenborg, and M. J. C. van Gemert. Double-integrating-sphere system for measuring the optical properties of tissue. Appl. Opt., 32(4):399, 1993.

[35] A. D. Klose and E. W. Larsen. Light transport in biological tissue based on the simplified spherical harmonics equations. J. Comput. Phys., 220(1):441, 2006.

[36] D. Dickey, O. Barajas, K. Brown, J. Tulip, and R. B. Moore. Radiance modelling using the p3 approximation. Phys. Med. Biol., 43(12):3559, 1998.

[37] D. J. Dickey, R. B. Moore, D. C. Rayner, and J. Tulip. Light dosimetry using the p3 approximation. Phys. Med. Biol., 46(9):2359, 2001. 
[38] T. Burger, J. Kuhn, R. Caps, and J. Fricke. Quantitative determination of the scattering and absorption coefficients from diffuse reflectance and transmittance measurements: Application to pharmaceutical powders. Appl. Spectrosc., 51(3):309, 1997.

[39] S. S. Sekulic, H. W. Ward, D. R. Brannegan, E. D. Stanley, C. L. Evans, S. T. Sciavolino, P. A. Hailey, and P. K. Aldridge. On-line monitoring of powder blend homogeneity by near-infrared spectroscopy. Anal. Chem., 68(3):509-513, 1996.

[40] R. R. Shinde, G. V. Balgi, S. L. Nail, and E. M. Sevick-Muraca. Frequency-domain photon migration measurements for quantitative assessment of powder absorbance: A novel sensor of blend homogeneity. J. Pharm. Sci., 88(10):959-966, 1999.

[41] C. F. Bohren and D. R. Huffman. Absorption and scattering of light by small particles. Wiley, New York, 1998.

[42] S. Chandrasekhar. Radiative Transfer. Dover Publications Inc., New York, 1960.

[43] A. Ishimaru. Wave propagation and scattering in random media. Academic, Vols. I and II, 1978.

[44] K. K. Sen and S. J. Wilson. Radiative Transfer in Curved Media: Basic Mathematical Methods for Radiative Transfer and Transport Problems in Participating Media of Spherical and Cylindrical Geometry. World Scientific, Singapore, 1990.

[45] A. Liemert and A. Kienle. Explicit solutions of the radiative transport equation in the p3 approximation. Med. Phys., 41(11), 2014.

[46] A. J. Welch and M. J. C. van Gemert. Optical-Response of Laser-Irradiated Tissue. Lasers, Photonics, and Electro-Optics. Springer US, 1995.

[47] Weber H. J. Arfken, G. B. Mathematical methods for physicists. Elsevier, Burlington, 2005. 
[48] R. Courant and D. Hilbert. Methods of Mathematical Physics. Wiley, New Jersey, 1989.

[49] R. E. Marshak. Note on the spherical harmonic method as applied to the milne problem for a sphere. Phys. Rev., 71:443, 1947.

[50] K. M. Case and P. F. Zweifel. Linear transport theory. Addison-Wesley series in nuclear engineering. Addison-Wesley, Massachusetts, 1967.

[51] G. W. Faris. P(N) approximation for frequency-domain measurements in scattering media. Appl. Opt., 44(11):2058, 2005.

[52] A. Liemert, D. Reitzle, and A. Kienle. Analytical solutions of the radiative transport equation for turbid and fluorescent layered media. Sci. Rep., 7:3819, 2017.

[53] W. L. Vos, T. W. Tukker, A. P. Mosk, A. Lagendijk, and W. L. IJzerman. Broadband mean free path of diffuse light in polydisperse ensembles of scatterers for white lightemitting diode lighting. Appl. Opt., 52(12):2602-2609, 2013.

[54] V. Y. F. Leung, A. Lagendijk, T. W. Tukker, A. P. Mosk, W. L. IJzerman, and W. L. Vos. Interplay between multiple scattering, emission, and absorption of light in the phosphor of a white light-emitting diode. Opt. Express, 22(7):8190, 2014.

[55] D. Malacara. Color vision and colorimetry: theory and applications. SPIE, Washington, 2011.

[56] M. C. W. van Rossum and Th. M. Nieuwenhuizen. Multiple scattering of classical waves: microscopy, mesoscopy, and diffusion. Rev. Mod. Phys., 71:313-371, 1999.

[57] P. D. Garcia, R. Sapienza, J. Bertolotti, M. D. Martin, A Blanco, A. Altube, L. Vina, D. S. Wiersma, and C. Lopez. Resonant light transport through mie modes in photonic glasses. Phys. Rev. A, 78:023823, 2008. 
[58] O. L. Muskens and A. Lagendijk. Broadband enhanced backscattering spectroscopy of strongly scatteringmedia. Opt. Express, 16(2):1222-1231, 2008.

[59] H. van de Hulst. Multiple Light Scattering. Academic Press, Leiden, 1980.

[60] A. Kokhanovsky. Light Scattering Reviews 9. Springer-Verlag, Berlin Heidelberg, second edition, 2015.

[61] N. Garcia, A. Z. Genack, and A. A. Lisyansky. Measurement of the transport mean free path of diffusing photons. Phys. Rev. B, 46:14475-14479, 1992.

[62] D. J. Durian. Penetration depth for diffusing-wave spectroscopy. Appl. Opt., $34(30): 7100-7105$.

[63] R. Elaloufi, R. Carminati, and J. J. Greffet. Time-dependent transport through scattering media: from radiative transfer to diffusion. J. Opt. A: Pure Appl. Opt., 4(5):S103, 2002.

[64] R. Sarma, A. Yamilov, S. F. Liew, M. Guy, and H. Cao. Control of mesoscopic transport by modifying transmission channels in opaque media. Phys. Rev. B, 92:214206, 2015.

[65] A. Lagendijk, R. Vreeker, and P. De Vries. Influence of internal reflection on diffusive transport in strongly scattering media. Phys. Lett. A, 136(1-2):81-88, 1989.

[66] J. Gómez Rivas, R. Sprik, C. M. Soukoulis, K. Busch, and A. Lagendijk. Optical transmission through strong scattering and highly polydisperse media. Europhys. Lett., 48(1):22, 1999.

[67] J. X. Zhu, D. J. Pine, and D. A. Weitz. Internal reflection of diffusive light in random media. Phys. Rev. A., 44:3948-3959, 1991.

[68] O. L. Muskens and A. Lagendijk. Method for broadband spectroscopy of light transport through opaque scattering media. Opt. Lett., 34(4):395-397, 2009. 
[69] See catalog at: http://www.lighting.philips.co.uk/pwc_li/gb_en/subsites/ oem/fortimo-led-catalogue. Accessed on 15 June 2015.

[70] K. Busch, C. M. Soukoulis, and E. N. Economou. Transport and scattering mean free paths of classical waves. Phys. Rev. B, 50:93-98, 1994.

[71] S. M. Kaczmarek, G. Domianiak-Dzik, W. Ryba-Romanowski, J. Kisielewski, and J. Wojtkowska. Changes in optical properties of ce: Yag crystals under annealing and irradiation processing. Cryst. Res. Technol., 34(8):1031-1036, 1999.

[72] G. J. Zhao, X. H. Zeng, J. Xu, S. M. Zhou, and Y. Z. Zhou. Temperature gradient technique (tgt) growth and characterizations of large-sized ce-doped yag scintillation crystal. Phys. Status Solidi A, 199(2):355-359, 2003.

[73] J. A. Mares, A. Beitlerova, M. Nikl, N. Solovieva, K. Nitsch, M. Kucera, M. Kubova, V. Gorbenko, and Y. Zorenko. Scintillation and optical properties of yag:ce films grown by liquid phase epitaxy. Radiat. Meas., 42(4-5):533 - 536, 2007.

[74] E. Mihokova, M. Nikl, A. Beitlerova, A. Vedda, K. Nejezchleb, K. Blazek, and C. D'Ambrosiod. Luminescence and scintillation properties of yag:ce single crystal and optical ceramics. J. Lumin., 126(1):77-80, 2007.

[75] M. Kučera, P. Hasa, and J. Hakenová. Optical and magneto-optical properties of ce:yag. J. Alloy Compd., 451(1-2):146 - 148, 2008.

[76] M. Bass, C. DeCusatis, J. Enoch, V. Lakshminarayanan, G. Li, C. Macdonald, V. Mahajan, and E. Van Stryland. Handbook of Optics, Third Edition Volume III: Vision and Vision Optics(Set). McGraw-Hill, Inc., New York, NY, USA, 3 edition, 2010.

[77] M. Kaveh. Analogies in Optics and Micro Electronics. Springer Netherlands, 1991. 
[78] E. D. Aydin, C. R. E. De Oliveira, and A. J. H. Goddard. A comparison between transport and diffusion calculations using a finite element-spherical harmonics radiation transport method. Med. Phys., 29(9):2013-2023, 2002.

[79] Lighttools. https://optics.synopsys.com/lighttools/. Accessed on 30 August 2017.

[80] C. J. Funk. Multiple scattering calculations of light propagation in ocean water. Appl. Opt., 12(2):301, 1973.

[81] E. P. Zege, 1. L. Katsev, and I. N. Polonsky. Multicomponent approach to light propagation in clouds and mists. Appl. Opt., 32(15):2803, 1993.

[82] G. R. Fournier and F. J. Luc. Analytic phase function for ocean water. In Analytic phase function for ocean water, volume 2258, page 194. Proc. SPIE, 1994.

[83] F. Fell and J. Fischer. Numerical simulation of the light field in the atmosphere-ocean system using the matrix-operator method. J. Quant. Spectros. Radiat. Transfer, 69(3):351, 2001.

[84] D. Stramski, E. Boss, D. Bogucki, and K. J. Voss. The role of seawater constituents in light backscattering in the ocean. Progr. Oceanogr., 61(1):27, 2004.

[85] D. A. Boas and H. Liu. Photon migration within the P3 approximation. In Proc. SPIE, volume 2389, page 240. SPIE, 1995.

[86] M. L. Meretska, A. Lagendijk, H. Thyrrestrup, A. P. Mosk, W. L. IJzerman, and W. L. Vos. How to distinguish elastically scattered light from stokes shifted light for solid-state lighting? J. Appl. Phys., 119(9):093102, 2016.

[87] M. Querry. Optical constants of minerals and other materials from the millimeter to the ultraviolet. Technical report, Defence Technical Information Center, 1987. 
[88] X. C. Li, J. M. Zhao, C. C. Wang, and L. H. Liu. Improved transmission method for measuring the optical extinction coefficient of micro/nano particle suspensions. Applied Optics, 55(29):8171, 2016.

[89] S. Mujumdar, R. Torre, H. Ramachandran, and D. Wiersma. Monte carlo calculations of spectral features in random lasing. JNP, 4(1):041550-041550-13, 2010.

[90] R. Uppu and S. Mujumdar. Dependence of the gaussian-lévy transition on the disorder strength in random lasers. Phys. Rev. A, 87:013822, 2013.

[91] H. A. Gaonkar, Dinesh K., Rajagopal R., and Arindam R. Decoupling scattering and absorption of turbid samples using a simple empirical relation between coefficients of the kubelka-munk and radiative transfer theories. Appl. Opt., 53(13):2892-2898, 2014.

[92] S. Rotter and S. Gigan. Light fields in complex media: Mesoscopic scattering meets wave control. Rev. Mod. Phys., 89:015005, 2017.

[93] Commission internationale de l'Eclairage proceedings. Cambridge University Press, Cambridge, 1930.

[94] T. Smith and J. Guild. The c.i.e. colourimetric standards and their use. Trans. Opt. Soc., 33:73, 1931.

[95] H. Luo, Jong K. K., E. F. Schubert, J. Cho, C. Sone, and Y. Park. Analysis of highpower packages for phosphor-based white-light-emitting diodes. Appl. Phys. Lett., $86(24): 243505,2005$.

[96] N. T. Tran and F. G. Shi. Studies of phosphor concentration and thickness for phosphor-based white light-emitting-diodes. J. Lightwave Technol., 26(21):3556-3559, 2008. 
[97] M. L. Meretska, R. Uppu, G. Vissenberg, A. Lagendijk, W. L. Ijzerman, and W. L. Vos. Analytical modeling of light transport in scattering materials with strong absorption. Opt. Express, 25(20):A906-A921, 2017. Chapter 4 .

[98] R. J. Crilly, W.-F. Cheong, B. Wilson, and J. R. Spears. Forward-adjoint fluorescence model: Monte carlo integration and experimental validation. Appl. Opt., 36(25):6513$6519,1997$.

[99] T. Oppenländer. Glossary of Terms Used in Photochemistry. Wiley-VCH Verlag GmbH and Co. KGaA, 2007.

[100] A. M. Brouwer. Standards for photoluminescence quantum yield measurements in solution (iupac technical report). Pure Appl. Chem., 83:2213-2228, 2011.

[101] G. Blasse and A. Bril. Investigation of Some Ce3+ Activated Phosphors. J. Chem. Phys., 47(12):5139-5145, 1967.

[102] H. Yang, G. Zhu, L. Yuan, C. Zhang, F. Li, H. Xu, and A. Yu. Characterization and luminescence properties of YAG: Ce 3+ phosphors by molten salt synthesis. J Am. Ceram. Soc., 95(1):49-51, 2012.

[103] D. J. Durian, D. A. Weitz, and D. J. Pine. Multiple light-scattering probes of foam structure and dynamics. Science, 252(5006):686-688, 1991.

[104] F. W. Taylor. Elementary Climate Physics. OUP Oxford, Oxford, 2005.

[105] Y. Takano and K.-N. Liou. Solar radiative transfer in cirrus clouds. part i: Singlescattering and optical properties of hexagonal ice crystals. J. Atmos. Sci., 46(1):3-19, 1989.

[106] W.-F. Cheong, S. A. Prahl, and A. J. Welch. A Review of the Optical Properties of Biological Tissues. IEEE J. Quantum Elect., 26(12):2166-2185, 1990. 
[107] C. A. Leatherdale, W.-K. Woo, F. V. Mikulec, and M. G. Bawendi. On the absorption cross section of cdse nanocrystal quantum dots. J. Phys. Chem. B, 106(31):7619-7622, 2002.

[108] Z. Shi and C. A. Anderson. Pharmaceutical applications of separation of absorption and scattering in near-infrared spectroscopy (nirs). J. Pharm. Sci., 99(12):4766-4783, 2010.

[109] M. U. Vera and D. J. Durian. Angular distribution of diffusely transmitted light. Phys. Rev. E, 53:3215-3224, 1996.

[110] P.-A. Lemieux, M. U. Vera, and D. J. Durian. Diffusing-light spectroscopies beyond the diffusion limit: The role of ballistic transport and anisotropic scattering. Phys. Rev. E, 57:4498-4515, 1998.

[111] M. J. C. van Gemert, R. Verdaasdonk, E. G. Stassen, G. A. C. M. Schets, G. H. M. Gijsbers, and J. J. Bonnier. Optical properties of human blood vessel wall and plaque. Lasers in Surgery and Medicine, 5(3):235-237, 1985.

[112] M. Keijzer, R. R. Richards-Kortum, S. L. Jacques, and M. S. Feld. Fluorescence spectroscopy of turbid media: Autofluorescence of the human aorta. Appl. Opt., 28(20):4286-4292, 1989.

[113] J. W. Goodman. Speckle phenomena in optics: theory and applications. Roberts and Company, Englewood, 2007.

[114] I. M. Vellekoop and A. P. Mosk. Phase control algorithms for focusing light through turbid media. Optics Communications, 281(11):3071 - 3080, 2008.

[115] O. S. Ojambati, J. T. Hosmer-Quint, K. J. Gorter, A. P. Mosk, and W. L. Vos. Controlling the intensity of light in large areas at the interfaces of a scattering medium. Phys. Rev. A, 94:043834, 2016. 
[116] I. M. Vellekoop and A. P. Mosk. Focusing coherent light through opaque strongly scattering media. Opt. Lett., 32(16):2309-2311, 2007.

[117] I. M. Vellekoop, E. G. van Putten, A. Lagendijk, and A. P. Mosk. Demixing light paths inside disordered metamaterials. Opt. Express, 16(1):67-80, 2008.

[118] K. L. van der Molen, A. P. Mosk, and A. Lagendijk. Intrinsic intensity fluctuations in random lasers. Phys. Rev. A, 74:053808, 2006.

[119] S. A. Goorden, J. Bertolotti, and A. P. Mosk. Superpixel-based spatial amplitude and phase modulation using a digital micromirror device. Opt. Express, 22(15):17999$18009,2014$.

[120] R. Lafer-Sousa, K. L. Hermann, and B. R. Conway. Striking individual differences in color perception uncovered by 'the dress' photograph. Current biology : $C B$, 25(13):R545-6, 2015.

[121] K. R. Gegenfurtner, M. Bloj, and M. Toscani. The many colours of 'the dress'. Current biology : $C B, 25(13):$ R543-4, 2015.

[122] J. K. Bowmaker and H. J. Dartnall. Visual pigments of rods and cones in a human retina. The Journal of physiology, 298:501-11, 1980.

[123] B. A. Wandell. Foundations of vision. Sinauer Assoc. Inc., Massachusetts, 1995. 


\section{Acknowledgments}

Mentors are the people who help us to advance and grow in our lives. I was lucky to have three mentors during my Ph.D., namely Willem Vos, Wilbert IJzerman, and Ad Lagendijk. Each of you is so different and at the same time so similar.

Willem taught me the backbone of the research. These are those small but crucial things that make challenging experiments to work. Willem gave me numerous advice on how to efficiently reach the goals in research and life. He regularly grilled me during the presentations and group talks. This grilling is precious, as he want us always to be at the top. Work-life balance is also something I can learn from you, as you take care of two amazing girls Johanna and Milja and your wife Acca. Of course, I must mention your fantastic ability to manage COPS. You always find an approach to every person in the group, see weak points, and make that person work on it. Thank you, Willem, for all these years. I learned a lot and enjoyed my Ph.D. journey with you being my mentor! I would not be myself if I did not join COPS led by you.

Wilbert provided me a valuable corporate experience. One can rarely get this experience during Ph.D. Thankfully to Wilbert I had several opportunities to directly communicate and present my work to the R\&D staff at Philips Lighting. These presentations taught me the lesson that industry is different, and I need to answer their questions directly and up to a point. For industry the most important question is to produce an excellent product, the details are secondary. Your advice and mentoring significantly helped me during my Ph.D. Thank you, Wilbert, for mentoring me on this journey!

Ad, you are a charismatic and remarkable person and scientist. I never met a person similar to you. For me, any problem is a double-edged sword. For you, there is always the third edge. You can unexpectedly look at the problems, that helps to understand it much better. Your famous "Survival guide for the scientist," provides an inexhaustible source of surviving methods in science and life. Advice learned from this book helped me not once. Career advice, and life lessons learned from you helped me during my Ph.D. and beyond. We still need to go together with ex-copses to the adventures. Thanks Ad for being a great 
supervisor and friend to me!

To summarize, I want to thank you guys for guiding me through this adventure. I enjoyed a lot these four years, and I am lucky to have you as my mentors. You are not good supervisors, you are excellent supervisors! You are the unique team of scientist that I had a pleasure to work with.

I would like to thank my committee members Klaus Boller, Jamie Gomez Rivas, Julius Muschaweck, Pepijn Pinkse, Rebecca Saive, Bart van Tiggelen to read my thesis carefully and being committee members for my defense.

I would like to thank Pepijn Pinkse for showing curiosity in my work and giving me useful tips regarding scientific work. Questions that you asked provided fruitful discussions that lead to new excellent additions to my papers. Allard Mosk was present only during half of my stay in COPS, but I learned many things from discussions with him. Our short communication with Jan Klärs and Rebecca Saive supplied me with tips and tricks for the young scientist. Thank you guys for sharing your experience with me. Bill Barnes is a guest in our group. Bill I am still hunting for the best cooking recipes from you. You were an excellent host during my visit in Exeter. I had a pleasure to meet and communicate with Ivo Vellekop. I received valuable life advice from you. I wish I had more time and opportunities to talk to you. Sonia García Blanco and Jennifer Herek for running important community Female Faculty Network Twente (FFNT). Thank you Sushil Mujumdar for hosting me in Mumbai, I had a pleasure to visit your lab. Sanli great that you agreed to be my paranyph, you are a great guy! You had an awesome dress during your defense.

I had a pleasure to work with Vanessa Leung, Henri Thyrrestrup Nielsen, Mehdi Aas. You were exceptional postdocs, maybe not with all of you I had a chance to overlap a lot, but you definitely shaped this project. Cock Harteveld always helped a lot in the lab, our office, and safeguarded our safety. Jan Jansen from Philips Lighting fabricated samples that basically shaped this thesis. Samples are the crucial part of any experiment, and you did a great job!

I would like to thank my students Peter Hooijschuur, Teus Dubbink, Konstantinos Ladovrechis, Pim Venderbosch and Wouter Fokkema. Mentoring is not only giving advice but also learning. I learned a lot from you guys! I would like to express special thank to Pim, as the chapter on wavefront shaping would not see the light without him. Being a part of ANP community allowed me to meet Pablo Munoz and Simen Martinussen. You were a great partner in organizing ANP meetings. You were always giving a hand in complicated scheduling situations. Thank you, guys! Special thank I would like to express for Luba Amitonova and Shakeeb bin Hasan for helping me with my proposal. 
Secretary is the boss. Because he/she decide who can meet the boss. Our secretaries Nilda, Jacqueline, Carla, and Nienke, thank you all for doing this hard job. Nicole special thank you for helping me with everything. You are so easy going person, and I can always rely on you. Marlon, thank you for the ICT support and moving stories about Curacao. I wish I could go there one day.

COPS is an excellent environment for scientific and non-scientific communication. I enjoyed being a part of this group. Diana, you were not only a great friend but also psychologist, party buddy, adventure colleague. I can not imagine that I could finish this trip without you. Many adventures and your ability to communicate improved me a lot. Thank you for being with me all this time. Femi Ojambati thank you for entertaining me for the most of my Ph.D. I will not forget your laugh, positive and ambitious attitude to life. Evangelos Marakis you contributed to my excess weight, but I can't stand the sweats you supplied. Tristan Tentrup thank you for being a soldier of COPS. You never refuse to serve in the name of science. Devashish Devashish thank you for an excellent company in the last days of your Ph.D. and help with English. I still owe you a beer, and now it is recorded in my thesis. Sjoerd you are an expert in swimming pools, and I got a lot of insight from you regarding this topic. It was a pleasure to work with Andy, Bas, Ben, Bert, Bill, Caterina, Chris, Emre, Georgios, Haider, Hasan, Henri, Iris, Jeroen, Julio, Jin, KlaasJan, Manashee, Matthijs, Oxan, Peilong, Pritam, Ravi, Raheleh, Reinier, Sergei, Takeyoshi, Thomas, Timmo, Tom, Vincent, Willemijn. It was a great pleasure to meet you guys.

My neighbors Ali, April, Frederik, Joost, Katharina, Meenali, Mieke, Raunak, Sasha always were fun, and they created a pleasant atmosphere in our house. Late night gaming and karaoke were enjoyable with you guys. I still have recordings of our "Dance Central" adventures. I would like to mention my friends with whom I contacted maybe not as often as I wanted during my Ph.D. We should have fun more often German, Ksusha, Sergei, Sasha.

I really improved my horseback riding skills thanks to Jitske and Daphne. I met a lot of friendly people that made my stay in Enschede fun. Among others, I would like to mention Arnoud, Dion, Doorle, Emma, Friso, Gijs, Ian, Ilja, Mirthe, Rolf, Sarah, Tessa, Vera, Wouter. It was a pleasure to meet people from Linea Recta. Thanks Tjerk for helping me during the training. I didn't have a chance to talk to you much, but I enjoyed every practice I had in Linea Recta. I met a great friend Vera and her partner Cesar there.

I would like to thank my family for supporting me for all this time. My father Leonid to whom I dedicate this thesis. He sparked in me the curiosity for science. He was a great teacher that had immense knowledge and erudition about everything in the world. 
At particular age kids start to ask questions continuously, which is sometimes is a problem for parents. For my father it was not a problem Rather I was tired of asking questions about everything. My mother Nataliia is like a mother-ship in Star Wars movie. Every time after I am punishing the "bad guys", I come back and receive the best treatment ever. The delicious food and infinite attention are invaluable. Thank you! In any critical moments in my life, I always knew that I can count on my extended family. They were always supporting me at all times. Thank you, Alina, Amina, Anna, Borus, Borus, Genya, Igor, Johan, Luda, Mariyka, Oksana, Oksana, Stas, Sweta, Valya, Victor.

I would like to thank Nono Groenen for supporting me during my Ph.D. journey and always asking me fundamental questions of Physics. You showed me the world of art. Since I met you, I have been exposed to all kind of exhibitions. Having you in my life is one of the best things happened to me! I am grateful that we have such good relationships. 